\title{
Preservação das classes de distribuições não-paramétricas \\ e desigualdades estocásticas entre os D-espectros de networks para seus respectivos tempos de vidas
}

Pedro Minoru Saito

\author{
DisSERTAÇÃO APRESENTADA \\ AO \\ Instituto de Matemática e Estatística \\ DA \\ Universidade DE SÃo PAUlo \\ PARA \\ OBTENÇÃO DO TÍTULO \\ $\mathrm{DE}$ \\ Mestre em CiÊnCIAS \\ Programa: Estatística \\ Orientador: Prof. Dr. Vanderlei da Costa Bueno
}

Durante o desenvolvimento deste trabalho o autor recebeu auxílio financeiro da CNPq

São Paulo, fevereiro de 2019 


\section{Preservação das classes de distribuições não-paramétricas \\ e desigualdades estocásticas \\ entre os D-espectros de networks para seus respectivos tempos de vidas}

Esta versão da dissertação contém as correções e alterações sugeridas pela Comissão Julgadora durante a defesa da versão original do trabalho, realizada em 22/02/2019. Uma cópia da versão original está disponível no Instituto de Matemática e Estatística da Universidade de São Paulo.

Comissão Julgadora:

- Prof ${ }^{a}$. Dra . Vanderlei da Costa Bueno - IME-USP

- Prof. Dr. Oswaldo Luiz do Valle Costa - EP-USP

- Prof. Dr. Cristian Favio Coletti - UFABC 


\section{Resumo}

SAITO, P. M. Preservação das classes de distribuições não-paramétricas e desigualdades estocásticas entre os D-espectros de networks para seus respectivos tempos de vidas. 2018. 84 f. Dissertação (Mestrado) - Instituto de Matemática e Estatística, Universidade de São Paulo, São Paulo, 2019.

Este trabalho reporta sobre a avaliação da confiabilidade de networks, uma representação analítica para diversos sistemas de engenharia e de comunicação, cujas falhas de seus componentes (links) ocorrem segundo um Processo de Poisson Não Homogêneo. Concluiremos que, na comparação de dois networks com a mesma quantidade de links, as desigualdades estocásticas de seus D-espectros serão preservadas em seus tempos de vidas e a preservação das classes de distribuições do D-espectro para o tempo de vida de um network ocorrerá com restrições na função de risco do Processo de Poisson Não Homogêneo.

Palavras-chave: confiabilidade, networks, processos de Poisson não homogêneo, desigualdades estocásticas, classes de distribuições não-paramétricas. 


\section{Abstract}

SOBRENOME, A. B. C. Preservation of D-spectra nonparametric distribution classes and stochastic inequalites to respective networks lifetimes. 2018. $84 \mathrm{f}$. Dissertação (Mestrado) Instituto de Matemática e Estatística, Universidade de São Paulo, São Paulo, 2019.

This work reports networks reliability evaluation, an analytic representation to several engineering and comunication systems, whose components (links) failures occur according to a Nonhomogeneous Poisson Process. We will conclude that, on comparison of two networks with same number of links, stochastic orders of their D-spectra will be preserved to their lifetimes and distribution classes preservation of network D-spectrum to its lifetime will occur with restrictions in hazard function of Nonhomogeneous Poisson Process.

Keywords: reliability, networks, nonhomogeneous Poisson processes, stochastic orders, nonparametric distributions classes. 


\section{Sumário}

$\begin{array}{ll}\text { Resumo } & \text { i }\end{array}$

$\begin{array}{lll}\text { Abstract } & \text { iii }\end{array}$

Lista de Abreviaturas vii

$\begin{array}{lc}\text { Lista de Figuras } & \text { ix }\end{array}$

1 Networks $\quad 1$

2 Resultados de preservação das desigualdades estocásticas $\quad 5$

2.1 Falhas segundo um Processo de Contagem . . . . . . . . . . . . . . . . 5

2.2 Resultados de equivalências de D-espectros para os D-espectros condicionais de networks

2.3 Preservações das desigualdades estocásticas entre D-espectro e tempo de vida de

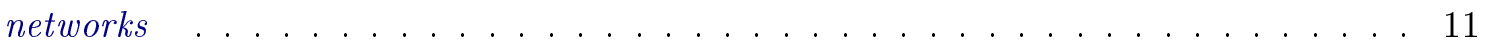

2.4 Resultados de Preservações entre D-espectros condicionais e tempos de vidas residuais 14

3 Resultados de preservação das classes de distribuições

3.1 Resultados de preservação de classes de distribuições do D-espectro para o tempo de vida de um network . . . . . . . . . . . . . . . . . . . . . . 19

3.2 Resultados de equivalências das classes de distribuições do D-espectro para o Despectro condicional de um network . . . . . . . . . . . . . . . . . . . 34

3.3 Resultados de preservação do D-espectro condicional para o tempo de vida de um

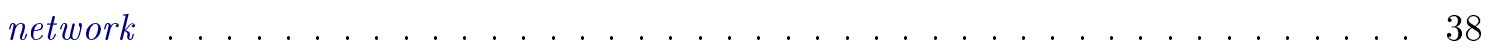

4 Conclusões $\quad 41$

4.1 Considerações Finais . . . . . . . . . . . . . . . . . . . . . . . . 41

4.2 Sugestões para Pesquisas Futuras . . . . . . . . . . . . . . . . . . . . . 41

$\begin{array}{ll}\text { A Apêndice } & 43\end{array}$

A.1 Sistemas coerentes e assinaturas . . . . . . . . . . . . . . . . 43

A.2 Desigualdades estocásticas . . . . . . . . . . . . . . . . . . 47

A.3 Classes de distribuições . . . . . . . . . . . . . . . . . . 50

A.4 Verificações para os Exemplos . . . . . . . . . . . . . . . 53

$\begin{array}{ll}\text { Referências Bibliográficas } & 71\end{array}$ 


\section{Lista de Abreviaturas}

DFR Taxa de falha decrescente (Decreasing Failure Rate)

DFRA Taxa de falha decrescente em média (Decreasing Failure Rate in Average)

HR Taxa de falha (Hazard Rate)

i.i.d. Independentes e identicamente distribuídos

IFR Taxa de falha crescente (Increasing Failure Rate)

IFRA Taxa de falha crescente em média (Increasing Failure Rate in Average)

LR Razão de verossimilhanças (Likelihood Ratio)

NBU Novo melhor do que usado (New better than used)

NBUE Novo melhor do que usado em média (New better than used in expectation)

NWU Novo pior do que usado (New worse than used)

NWUE Novo pior do que usado em média (New worse than used in expectation)

PPH Processo de Poisson Homogêneo

PPNH Processo de Poisson Não Homogêneo 


\section{Lista de Figuras}

1.1 Figura 1.1. Network com 4 links, 4 nós e 3 terminais . . . . . . . . . . . . . . 2

A.1 Figura A.1.1. Sistema tipo série-paralelo com 3 componentes . . . . . . . . . . . . . 45 


\section{Capítulo 1}

\section{Networks}

Um network é uma coleção de nós e links, na qual alguns nós são chamados terminais. Representa uma variedade de sistemas da vida real na engenharia, indústria e comunicação. Exemplos de nós são: intersecções rodoviárias, estações de telecomunicações, servidores e computadores e exemplos de links são: rodovias, fibras de telecomunicações, cabos e canais sem fio.

De acordo com a literatura existente, um network pode ser modelado por uma tripla $\mathbf{N}=(\mathrm{V}, \mathrm{E}, \mathrm{T})$, na qual, $\mathrm{V}$ é o conjunto dos nós com cardinalidade $|\mathrm{V}|=\mathrm{m}$, E é o conjunto dos links com cardinalidade $|\mathrm{E}|=\mathrm{n}$, e $\mathrm{T} \subseteq \mathrm{V}$ é o conjunto dos terminais. Nesta dissertação, assumimos que os nós são determinísticos e que os links são aleatórios, sujeitos a falhas segundo um mecanismo estocástico. A falha de um link significa que este é removido e pode resultar na troca de estado do network. Quando todos os terminais do network estão conectados entre si, o network é denominado T-conectado.

Uma abordagem clássica do network é sua interpretação como um sistema coerente e, como tal, interpretamos seus links como componentes sujeitos a falhas estocásticas. Neste trabalho, o network e links têm dois estados: o estado de funcionamento (1) e o estado de falha (0).

Para analisar a confiabilidade de um network com n links os quais possuem tempos de vidas $T_{1}, T_{2}, \ldots, T_{n}$ independentes e identicamente distribuídos, com função de distribuição contínua $\mathrm{G}$ e tempo de vida T, um conceito útil e importante é seu espectro de destruição (D-espectro), que depende de sua estrutura. Para entender o conceito do D-espectro primeiramente definimos uma âncora.

Definição 1.1. (Zarezadeh et al., 2013) Considere um network com n links. Seja $\pi=$ $\left(e_{i_{1}}, e_{i_{2}}, \ldots, e_{i_{n}}\right)$ uma permutação dos números dos links do network. Inicialmente suponha que todos os links da permutação $\pi$ estejam funcionando, em seguida percorre-se toda a permutação $\pi$ da esquerda para a direita e considere a falha de cada um dos links um após o outro. Tome $e_{i_{r}}$, $\mathrm{r}=1,2, \ldots, \mathrm{n}$, como o primeiro elemento quando network muda seu estado para falha. O número de falhas sequenciais de links necessárias para a falha do network, denotado por $r(\pi)$, é denominado âncora de $\pi$, ou seja, $r(\pi)=r$.

Como os tempos de vida dos links são independentes e identicamente distribuídos com função de distribuição contínua $\mathrm{G}$, temos que cada permutação $\pi$ tem probabilidade $P(\pi)=\frac{1}{n !}$. Se $n_{r}$ é a quantidade de casos com $\mathrm{r}$ falhas, para essa hipótese, com $\mathrm{r}=1, \ldots, \mathrm{n}$ :

$$
P(r(\pi)=r)=\frac{n_{r}}{n !}
$$


Com tais considerações, apresentamos a definição do D-espectro.

Definição 1.2. - ver Definição 1.1 de Zarezadeh et al. (2013) Considere um network com tempo de vida $\mathrm{T}$ e $\mathrm{n}$ links, com tempos de vida $T_{1}, \ldots T_{n}$, independentes e identicamente distribuídos e com função de distribuição contínua G. Seja $\pi$ uma das permutações das falhas de seus links e $\mathrm{r}(\pi)$ sua âncora. O D-espectro do network é definido pelo vetor $\mathbf{f}=\left(f_{1}, f_{2}, \ldots, f_{n}\right)$, onde

$$
f_{i}=P\left(T=T_{(i)}\right)=P(r(\pi)=i)=\frac{n_{i}}{n !},
$$

e $n_{i}$ é o número de permutações que causam a falha do network na i-ésima falha dos links, $\mathrm{i}=1,2, \ldots, \mathrm{n}$, que constitui uma função discreta de probabilidade.

O D-espectro acumulado (Zarezadeh e Asadi, 2013) é sua função de distribuição definida por

$$
F_{i}=\sum_{k=1}^{i} f_{k} .
$$

Esta medida é a função de distribuição do número de falhas dos links necessárias para causar a falha do network.

E sua função de sobrevivência (Zarezadeh e Asadi, 2013) é

$$
\bar{F}_{i}=\sum_{k=i+1}^{n} f_{k}
$$

que é a probabilidade do network sobreviver a uma determinada quantidade de falhas de seus links.

Exemplo 1.1. (ver Exemplo 2.1 de Zarezadeh et al. (2013)) Considere um network com 4 links, 4 nós e 3 terminais, conforme a Figura 1.1. Assuma que todos os links estão sujeitos a falhas. O network funciona se e só se todos os terminais estiverem conectados um ao outro. Suponha que cada permutação dos links tenha a mesma probabilidade de ocorrência.

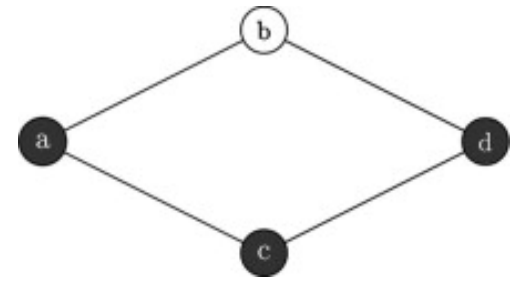

Figura 1.1: Figura 1.1. Network com 4 links, 4 nós e 3 terminais

Para o cálculo do D-espectro deste network, utilizamos a Definição 1.2 deste trabalho. Para resolver este caso disponibilizamos de um total de 4! permutações dos links, isto é, 24 permutações. Se avaliarmos o network desligando um link por vez, percebemos que os nós continuam conectados um ao outro, mesmo indiretamente, isto é, não estando próximos entre si. Portanto, a falha de um link não é suficiente para causar a falha do network. Assim, $f_{1}=0$.

Para que o network interrompa seu funcionamento com três falhas de seus links é suficiente que ocorra uma das seguintes desconexões sucessivas entre os links: $\{a b, b d, a c\},\{a b, b d, c d\}$, $\{b d, a b, a c\},\{b d, a b, c d\}$. Portanto: $f_{3}=\frac{4}{24}=\frac{1}{6}$. 
São necessárias no máximo três falhas dos links para interromper o funcionamento do network. Portanto: $f_{4}=0$.

Como o D-espectro é uma distribuição de probabilidade, temos:

$$
f_{1}+f_{2}+f_{3}+f_{4}=1 \Longleftrightarrow 0+f_{2}+\frac{1}{6}+0=1 \Longleftrightarrow f_{2}=\frac{5}{6} .
$$

O D-espectro do network é $\mathbf{f}=\left(0, \frac{5}{6}, \frac{1}{6}, 0\right)$. Pela representação (1.3), a função de sobrevivência do D-espectro é $\overline{\mathbf{F}}=\left(1,1, \frac{1}{6}, 0\right)$. Conforme a relação (1.2) a função de distribuição acumulada vale: $\mathbf{F}=\left(0, \frac{5}{6}, 1,1\right)$.

Vale mencionar que o D-espectro definido acima é idêntico ao vetor de assinaturas (Definição A.1.3), largamente utilizado no estudo de sistemas coerentes (Definição A.1.2).

Recentemente vários autores como Gertsbakh e Shpungin (2011), Samaniego et al. (2009), Balakrishnan e Asadi (2012), Boland et al. (2003) e outros analisam a confiabilidade do network baseada no conceito de assinaturas com a suposição de que os tempos de vidas dos links são independentes e identicamente distribuídos com função de distribuição contínua G.

Autores como Esary et al. (1973) e A-Hameed e Proschan (1973) analisam a confiabilidade de um dispositivo sujeito a choques em seus componentes, no entanto as caracterizações da confiabilidade deste dispositivo e da probabilidade de sobrevivência a choques são idênticas à confiabilidade de um network e à função de sobrevivência do D-espectro, respectivamente e úteis nas provas dos teoremas de preservações das classes de distribuições. No entanto, Esary et al. (1973) analisam a confiabilidade de um dispositivo com componentes sujeitos a choques que ocorrem segundo um Processo de Poisson Homogêneo e A-Hameed e Proschan (1973) o fazem quando se tem um Processo de Poisson Não Homogêneo.

No entanto, para definir o D-espectro é suficiente assumir que as ordenações das falhas dos links têm igual probabilidade. Desta forma o D-espectro de um network não depende das distribuição dos tempos de vidas de seus links, e sim de sua estrutura, que também é uma característica do conceito de assinaturas (Samaniego et al., 2009).

Nesta dissertação nosso objetivo é interpretar um network como um sistema coerente, e através de seu D-espectro (assinaturas) calcular sua confiabilidade, estudar propriedades de preservação das desigualdades estocásticas entre D-espectros para os tempos de vidas de seus respectivos networks, e das classes de distribuições do D-espectro para o tempo de vidas do respectivo network. Estes últimos resultados não aparecem formalmente na literatura sobre a confiabilidade de networks.

Em particular, assumimos que as ordenações das falhas dos links são igualmente prováveis e que as falhas ocorrem segundo um Processo de Poisson Não Homogêneo (PPNH).

Neste caso é interessante notar que a confiabilidade de um network cujas falhas dos links ocorrem segundo um processo de contagem é representada por uma mistura das confiabilidades dos primeiros $\mathrm{n}$ records de uma sequência de variáveis aleatórias não-negativas, independentes e identicamente distribuídas (vide Arnold et al. (1998) e Gupta e Kirman (1988)). Essa representação é análoga à forma de mistura da confiabilidade de um sistema coerente com n componentes em termos das confiabilidades das estatísticas de ordem de uma amostra de tamanho n, como nas assinaturas (Definição A.1.2).

Em resumo, considere um network cujas falhas de seus links ocorrem segundo um Processo de Poisson Não Homogêneo $\{N(t): t>0\}$, com média $\Lambda(t)$. Denote por $S_{k}$ o instante da k-ésima falha 
do processo, com $\mathrm{k}=0,1,2 \ldots$ Sua confiabilidade no instante $\mathrm{t}>0$ é

$$
\bar{G}_{k}(t)=P\left(S_{k}>t\right)=P(N(t)<k)=\sum_{i=0}^{k-1} \frac{e^{-\Lambda(t)} \cdot \Lambda(t)^{i}}{i !},
$$

onde $\Lambda(t)=E[N(t)]=-\ln (\bar{G}(t))$ é o número médio de ocorrências do processo, também denominado função de risco no instante t, e $\bar{G}(t)$ a confiabilidade do instante da primeira ocorrência.

Como em Arnold et al. (1998) e Gupta e Kirman (1988), a confiabilidade $\bar{G}_{k}(t)$ resulta naturalmente da confiabilidade dos records superiores como segue:

Considere uma sequência de variáveis aleatórias $Y_{1}, Y_{2}, \ldots$ independentes e identicamente distribuídas com função de distribuição contínua $G$, representando os tempos de vidas dos links do network.

Com base nestes tempos de vidas, define-se uma sequência de records $\{U(n), n=1,2, \ldots\}$, com:

$$
\begin{gathered}
U(1)=1 ; \\
U(n+1)=\min \left\{j: j>U(n), Y_{j}>Y_{U(n)}\right\}, n \geq 1 .
\end{gathered}
$$

Essa sequência de records está relacionada a uma nova ordenação de índices das falhas dos links. Definimos a sequência $\left\{R_{k}, k \geq 1\right\} \operatorname{com} R_{k}=Y_{U(k)}$, ou seja, a observação $R_{k}$ ultrapassa as observações anteriores. Se g é a função densidade de probabilidade da função de distribuição $G$, a função densidade de probabilidade de $R_{k}$ é

$$
g_{k}(t)=g(t) \frac{\Lambda(t)^{k-1}}{(k-1) !}, \mathrm{t}>0, \mathrm{k}=1,2, \ldots
$$

Isso mostra que em um PPNH com função de risco $\Lambda(t)=-\ln \bar{G}(t), R_{k}$ e $S_{k}$ têm a mesma distribuição. Ou seja, um Processo de Poisson Não Homogêneo é um processo de contagem não explosivo onde a sequência dos instantes de ocorrências são os records de variáveis aleatórias independentes e identicamente distribuídas com função de distribuição $\mathrm{G}$, isto é, o instante da k-ésima falha dos links de um network, com ocorrências segundo um PPNH, com $\Lambda(t)=-\ln (\bar{G}(t))$, é $R_{k}$, para $\mathrm{k}=0,1,2, \ldots \mathrm{n}$.

Nesta dissertação, no Capítulo 2, analisamos resultados de preservações das desigualdades estocásticas entre D-espectro para seus respectivos networks.

No Capítulo 3, estudamos resultados de preservação de classes de distribuições não paramétricas dos D-espectros para os respectivos networks.

As verificações algébricas dos exemplos deste trabalho encontram-se na Seção A.4.

Além da sistematização dos resultados apresentados, uma das principais contribuições do autor foi a de exemplificar ou contra-exemplificar os resultados provados. 


\section{Capítulo 2}

\section{Resultados de preservação das desigualdades estocásticas}

Antes de analisarmos a preservação das desigualdades estocásticas contextualizaremos o processo de contagem para as falhas dos links de um network.

\subsection{Falhas segundo um Processo de Contagem}

Considere um network com tempo de vida $\mathrm{T}, \mathrm{n}$ links, e D-espectro $\mathbf{f}$, onde as ordenações das falhas são igualmente prováveis e ocorrem segundo um processo de contagem $\{N(t): t>0\}$. Seja $S_{k}, \mathrm{k}=1,2 \ldots \mathrm{n}$, o instante da k-ésima falha. Pelo Teorema 2.1 de Zarezadeh e Asadi (2013), a confiabilidade do network no instante t $>0$ é

$$
\begin{gathered}
\bar{H}(t)=P(T>t)=\sum_{k=1}^{n} P\left(T>t \mid T=S_{k}\right) P\left(T=S_{k}\right) \\
=\sum_{k=1}^{n} P\left(S_{k}>t \mid T=S_{k}\right) P\left(T=S_{k}\right) .
\end{gathered}
$$

Como as ordenações das falhas dos componentes são igualmente prováveis, os eventos $\left\{T=S_{k}\right\}$ e $\left\{S_{k}>t\right\}$ são independentes (Lema A.1.1). Portanto

$$
\bar{H}(t)=\sum_{k=1}^{n} f_{k} \cdot P\left(S_{k}>t\right) .
$$

Alternativamente a confiabilidade de um network é

$$
\begin{aligned}
\bar{H}(t) & =\sum_{k=1}^{n} f_{k} \cdot P\left(S_{k}>t\right)=\sum_{k=1}^{n} f_{k} \cdot P(N(t)<k)=\sum_{k=1}^{n} \sum_{j=0}^{k-1} f_{k} \cdot P(N(t)=j) \\
& =\sum_{j=0}^{n-1}\left(\sum_{k=j+1}^{n} f_{k}\right) \cdot P(N(t)=j)=\sum_{j=0}^{n-1} \bar{F}_{j} \cdot P(N(t)=j)=E\left[\bar{F}_{N(t)}\right] .
\end{aligned}
$$


A função densidade do tempo de vida de um network no instante t $>0$ é

$$
h(t)=-\frac{d}{d t} \bar{H}(t)=-\frac{d}{d t} \sum_{k=1}^{n} f_{k} \cdot P\left(S_{k}>t\right)=\sum_{k=1}^{n} f_{k} \cdot g_{k}(t)
$$

onde $g_{k}(t)$ é a função densidade de probabilidade do instante da k-ésima falha, $\mathrm{k}=1,2, \ldots, \mathrm{t}>0$.

As representações da confiabilidade do network e da função densidade de probabilidade de seu tempo de vida são calculadas por Zarezadeh e Asadi (2013).

Para esta dissertação consideramos como processo de contagem das falhas dos links do network em estudo um Processo de Poisson Não Homogêneo.

Para analisar um network com tempo de vida T, na dinâmica do tempo, consideramos o tempo de vida residual $\{T-t \mid T>t\}$ e definimos o D-espectro condicional (Zarezadeh et al., 2013).

Seja $\mathbf{f}$ o D-espectro de um network cujas falhas ocorrem segundo um processo de contagem $\{N(t): t>0\}$. Considere o instante da k-ésima falha $S_{k}, \mathrm{k}=1, \ldots, \mathrm{n}$. A confiabilidade do tempo de vida residual no instante $\mathrm{x}>0$, dado que o network sobreviveu ao instante $\mathrm{t}>0$, é

$$
P(T-t>x \mid T>t)=\frac{P(T>x+t)}{P(T>t)}=\frac{\sum_{k=1}^{n} f_{k} P\left(S_{k}>x+t\right)}{\sum_{j=1}^{n} f_{j} P\left(S_{j}>t\right)}=\frac{\sum_{k=1}^{n} f_{k} P\left(S_{k}>x+t, S_{k}>t\right)}{\sum_{j=1}^{n} f_{j} P\left(S_{j}>t\right)} .
$$

Esta última igualdade ocorre pois o evento $\left\{S_{k}>t+x\right\}$ está contido no evento $\left\{S_{k}>t\right\}$. Além disso, pela definição de probabilidade condicional, $P\left(S_{k}>t+x, S_{k}>t\right)=P\left(S_{k}>t\right) \cdot P\left(S_{k}>\right.$ $\left.t+x \mid S_{k}>t\right)$, portanto:

$$
\begin{aligned}
P(T-t>x \mid T & >t)=\frac{\sum_{k=1}^{n} f_{k} P\left(S_{k}>t\right) \cdot P\left(S_{k}>t+x \mid S_{k}>t\right)}{\sum_{j=1}^{n} f_{j} P\left(S_{j}>t\right)} \\
& =\sum_{k=1}^{n} p_{k}(t) \cdot P\left(S_{k}>t+x \mid S_{k}>t\right),
\end{aligned}
$$

onde

$$
p_{k}(t)=\frac{f_{k} P\left(S_{k}>t\right)}{\sum_{j=1}^{n} f_{j} P\left(S_{j}>t\right)} .
$$

Interpretamos que a confiabilidade residual de um network que sobreviveu ao instante t é uma mistura das confiabilidades residuais de seus links que sobreviveram ao instante t.

Definição 2.1.1 Ao vetor $\mathbf{p}(\mathbf{t})=\left(p_{1}(t), \ldots, p_{n}(t)\right)$ denominamos D-espectro condicional no instante t.

Uma interpretação para o k-ésimo componente do D-espectro condicional é a probabilidade do sistema falhar na k-ésima falha de seus links, dado que o network sobreviveu ao instante t, pois

$$
P\left(T=S_{k} \mid T>t\right)=\frac{P\left(T=S_{k}, T>t\right)}{P(T>t)}=\frac{P\left(T=S_{k}, S_{k}>t\right)}{P(T>t)}=\frac{P\left(T=S_{k}\right) \cdot P\left(R_{k}>t \mid T=S_{k}\right)}{P(T>t)} .
$$

Como assumimos que as ordenações das falhas dos componentes são igualmente prováveis, podemos dizer que o evento $\left\{S_{k}>t\right\}$ é independente do evento $\left\{T=S_{k}\right\}$ (Lema A.1.1). 
Consequentemente

$$
P\left(T=S_{k} \mid T>t\right)=\frac{P\left(T=S_{k}\right) \cdot P\left(S_{k}>t\right)}{P(T>t)}=\frac{f_{k} \cdot P\left(S_{k}>t\right)}{\sum_{j=1}^{n} f_{j} P\left(S_{j}>t\right)}=p_{k}(t)
$$

O D-espectro condicional, a interpretação dos respectivos termos e a confiabilidade residual do respectivo network dado que este sobreviveu ao instante t são verificados em Zarezadeh et al. (2013).

As representações da confiabilidade e da função densidade de probabilidade associadas a um network e seu tempo de vida residual são suficientes para estudar a preservação das desigualdades estocásticas entre os D-espectros de networks para seus tempos de vidas e a preservação das classes de distribuições do D-espectro para o tempo de vida do respectivo network.

Frequentemente estamos interessados em comparar a confiabilidade de dois networks através de desigualdades estocásticas, tais como a ordenação estocástica (ST), a ordenação através das taxas de falhas (HR) e a ordenação por razão de verossimilhanças (LR) citadas em ordem de relevância crescente (Definições A.2.1, A.2.2, A.2.3).

\subsection{Resultados de equivalências de D-espectros para os D-espectros condicionais de networks}

Veremos um exemplo de resultados de equivalências de desigualdades estocásticas entre Despectros e D-espectros condicionais de seus respectivos networks. Este exemplo é de autoria própria neste trabalho.

Exemplo 2.2.1 (vide Exemplo A.4.1) Sejam $\mathbf{f}_{\mathbf{1}}=(0,5 ; 0,2 ; 0,3)$ e $\mathbf{f}_{\mathbf{2}}=(0,4 ; 0,2 ; 0,4)$ os D-espectros de networks, cujas falhas de seus links ocorrem segundo um Processo de Poisson Homogêneo com taxa $\lambda=2$ falhas/hora. Queremos analisar a equivalência das desigualdades estocásticas entre seus D-espectros e os D-espectros condicionais. Temos:

\begin{tabular}{|c|c|c|c|c|c|c|}
\hline $\mathrm{k}$ & $f_{1 k}$ & $f_{2 k}$ & $\bar{F}_{1 k}$ & $\bar{F}_{2 k}$ & $\frac{F_{2 k}}{F_{1 k}}$ & $\frac{f_{2 k}}{f_{1 k}}$ \\
\hline 0 & 0,5 & 0,4 & 1 & 1 & 1 & 0,8 \\
\hline 1 & 0,2 & 0,2 & 0,5 & 0,6 & 1,2 & 1,0 \\
\hline 2 & 0,3 & 0,4 & 0,3 & 0,4 & 1,3 & 1,3 \\
\hline
\end{tabular}

Com base na tabela acima, concluímos que $\mathbf{f}_{\mathbf{1}} \leq_{S T} \mathbf{f}_{\mathbf{2}}, \mathbf{f}_{\mathbf{1}} \leq_{H R} \mathbf{f}_{\mathbf{2}}$ e $\mathbf{f}_{\mathbf{1}} \leq_{L R} \mathbf{f}_{\mathbf{2}}$, respectivamente sob as Definições A.2.1, A.2.2 e A.2.3, respectivamente.

Mostraremos que as mesmas desigualdades valem para o D-espectro condicional:

$$
\begin{gathered}
P\left(T_{1}>t\right)=e^{-2 t}\left(1+t+0,6 t^{2}\right) ; \\
P\left(T_{2}>t\right)=e^{-2 t}\left(1+1,2 . t+0,8 t^{2}\right) .
\end{gathered}
$$

Observe que

$$
\begin{gathered}
P\left(R_{1}>t\right)=e^{-2 t} ; \\
P\left(R_{2}>t\right)=e^{-2 t}(1+2 t) ; \\
P\left(R_{3}>t\right)=e^{-2 t}\left(1+2 t+2 t^{2}\right) .
\end{gathered}
$$


O k-ésimo componente do D-espectro condicional do i-ésimo network, k=1,2,...n e i=1,2, dado $\left\{T_{i}>t\right\}$ é

$$
p_{i k}(t)=\frac{f_{i k} \cdot P\left(R_{k}>t\right)}{P\left(T_{i}>t\right)}
$$

Portanto:

$$
\begin{gathered}
p_{11}(t)=\frac{0,5}{\left(1+t+0,6 t^{2}\right)} ; \\
p_{12}(t)=\frac{0,2+0,4 t}{\left(1+t+0,6 t^{2}\right)} ; \\
p_{13}(t)=\frac{\left.0,3+0,6 . t+0,6 . t^{2}\right)}{\left(1+t+0,6 t^{2}\right)} ; \\
p_{21}(t)=\frac{0,4}{\left(1+1,2 . t+0,8 . t^{2}\right)} ; \\
p_{22}(t)=\frac{0,2+0,4 \cdot t}{\left(1+1,2 . t+0,8 . t^{2}\right)} ; \\
p_{23}(t)=\frac{0,4+0,8 . t+0,8 . t^{2}}{\left(1+1,2 . t+0,8 . t^{2}\right)}
\end{gathered}
$$

Consequentemente

$$
\begin{gathered}
\sum_{j=2}^{3} p_{1 j}(t)=\frac{0,5+t+0,6 . t^{2}}{\left(1+t+0,6 t^{2}\right)} \\
\sum_{j=3}^{3} p_{1 j}(t)=\frac{\left.0,3+0,6 . t+0,6 . t^{2}\right)}{\left(1+t+0,6 t^{2}\right)} ; \\
\sum_{j=2}^{3} p_{2 j}(t)=\frac{0,6+1,2 . t+0,8 . t^{2}}{\left(1+1,2 . t+0,8 . t^{2}\right)} \\
\sum_{j=3}^{3} p_{2 j}(t)=\frac{0,4+0,8 . t+0,8 . t^{2}}{\left(1+1,2 . t+0,8 . t^{2}\right)}
\end{gathered}
$$

onde $\sum_{j=k}^{n} p_{i j}(t)$ é a função de sobrevivência do D-espectro condicional do i-ésimo network a $\mathrm{k}$ falhas dos links no instante $\mathrm{t}, \mathrm{i}=1,2, \mathrm{k}=1, \ldots \mathrm{n}$.

Verificamos a equivalência da ordenação estocástica para o D-espectro condicional, ou seja, mostraremos que

$$
\sum_{j=k}^{3} p_{2 j}(t)-\sum_{j=k}^{3} p_{1 j}(t) \geq 0, \quad k=2,3 .
$$

Temos:

$$
\begin{aligned}
& \sum_{j=2}^{3} p_{2 j}(t)-\sum_{j=2}^{3} p_{1 j}(t)=\frac{0,1+0,2 \cdot t+0,16 \cdot t^{2}}{\left(1+t+0,6 t^{2}\right) \cdot\left(1+1,2 \cdot t+0,8 \cdot t^{2}\right)} \geq 0 \\
& \sum_{j=3}^{3} p_{2 j}(t)-\sum_{j=3}^{3} p_{1 j}(t)=\frac{(0,1+0,04 \cdot t) \cdot\left(1+2 t+2 t^{2}\right)}{\left(1+t+0,6 t^{2}\right) \cdot\left(1+1,2 \cdot t+0,8 \cdot t^{2}\right)} \geq 0 .
\end{aligned}
$$


Concluímos que $\mathbf{p}_{\mathbf{1}}(\mathbf{t}) \leq_{S T} \mathbf{p}_{\mathbf{2}}(\mathbf{t})$, sob a Definição A.2.1.

Verificaremos a equivalência da desigualdade através das taxas de falhas. Temos:

$$
\begin{gathered}
\frac{\sum_{j=1}^{3} p_{2 j}(t)}{\sum_{j=1}^{3} p_{1 j}(t)}=1 \\
\frac{\sum_{j=2}^{3} p_{2 j}(t)}{\sum_{j=2}^{3} p_{1 j}(t)}=1+\frac{0,1+0,2 . t+0,12 . t^{2}}{0,5+1,6 . t+2,2 . t^{2}+1,52 . t^{3}+0,48 . t^{4}} \\
\frac{\sum_{j=3}^{3} p_{2 j}(t)}{\sum_{j=3}^{3} p_{1 j}(t)}=1+\frac{1+0,4 . t}{3+3,6 . t+2,4 . t^{2}} .
\end{gathered}
$$

Observe que

$\frac{\sum_{j=3}^{3} p_{2 j}(t)}{\sum_{j=3}^{3} p_{1 j}(t)}-\frac{\sum_{j=2}^{3} p_{2 j}(t)}{\sum_{j=2}^{3} p_{1 j}(t)}=\frac{0,2+0,84 . t+1,52 . t^{2}+1,488 . t^{3}+0,8 . t^{4}+0,192 t^{5}}{\left(3+3,6 . t+2,4 . t^{2}\right)\left(0,5+1,6 . t+2,2 . t^{2}+1,52 . t^{3}+0,48 . t^{4}\right)} \geq 0$.

Concluímos que $\frac{\sum_{j=1}^{3} p_{2 j}(t)}{\sum_{j=1}^{3} p_{1 j}(t)} \leq \frac{\sum_{j=2}^{3} p_{2 j}(t)}{\sum_{j=2}^{3} p_{1 j}(t)} \leq \frac{\sum_{j=3}^{3} p_{2 j}(t)}{\sum_{j=3}^{3} p_{1 j}(t)}$ e $\mathbf{p}_{\mathbf{1}}(\mathbf{t}) \leq_{H R} \mathbf{p}_{\mathbf{2}}(\mathbf{t})$, sob a Definição A.2.2.

Avaliaremos a equivalência para as desigualdades através da razão de verossimilhanças.

Temos:

$$
\begin{aligned}
\frac{p_{21}(t)}{p_{11}(t)} & =\frac{0,8 \cdot\left(1+t+0,6 . t^{2}\right)}{1+1,2 . t+0,8 . t^{2}} \\
\frac{p_{22}(t)}{p_{12}(t)} & =\frac{1+t+0,6 . t^{2}}{1+1,2 . t+0,8 . t^{2}} \\
\frac{p_{23}(t)}{p_{13}(t)} & =\frac{4 .\left(1+t+0,6 . t^{2}\right)}{3 \cdot\left(1+1,2 . t+0,8 \cdot t^{2}\right)}
\end{aligned}
$$

Concluímos que $\frac{p_{21}(t)}{p_{11}(t)} \leq \frac{p_{22}(t)}{p_{12}(t)} \leq \frac{p_{23}(t)}{p_{13}(t)}$ e $\mathbf{p}_{\mathbf{1}}(\mathbf{t}) \leq_{\mathbf{L R}} \mathbf{p}_{\mathbf{2}}(\mathbf{t})$, sob a Definição A.2.3.

O exemplo mostra a equivalência das desigualdades estocásticas entre D-espectros e D-espectros condicionais. Essa equivalência é sempre verdadeira como prova o Teorema 2.3.1. A prova deste teorema é relevante para verificarmos que a comparação estocástica entre os D-espectros de seus networks nos basta para compararmos estocasticamente os respectivos D-espectros condicionais na dinâmica do tempo. Este teorema e a sua prova são do próprio autor.

Teorema 2.2.1 Sejam $\mathbf{f}_{\mathbf{1}}$ e $\mathbf{f}_{\mathbf{2}}$ os D-espectros de dois networks com n links, cujas falhas ocorrem segundo um mesmo Processo de Poisson Não Homogêneo $\{N(t): t>0\}$, com função de risco $\Lambda(t), t>0$. Sejam $\mathbf{p}_{\mathbf{1}}(\mathrm{t})$ e $\mathbf{p}_{\mathbf{2}}(\mathrm{t}), \mathrm{t}>0$, os respectivos D-espectros condicionais no instante $\mathrm{t}$.
(a) $\mathbf{f}_{\mathbf{1}} \leq_{S T} \mathbf{f}_{\mathbf{2}} \Longleftrightarrow \mathbf{p}_{\mathbf{1}}(\mathrm{t}) \leq_{S T} \mathbf{p}_{\mathbf{2}}(\mathrm{t}), \forall t>0$
(b) $\mathbf{f}_{\mathbf{1}} \leq_{H R} \mathbf{f}_{\mathbf{2}} \Longleftrightarrow \mathbf{p}_{\mathbf{1}}(\mathrm{t}) \leq_{H R} \mathbf{p}_{\mathbf{2}}(\mathrm{t}), \forall t>0$;
(c) $\mathbf{f}_{\mathbf{1}} \leq_{L R} \mathbf{f}_{\mathbf{2}} \Longleftrightarrow \mathbf{p}_{\mathbf{1}}(\mathrm{t}) \leq_{L R} \mathbf{p}_{\mathbf{2}}(\mathrm{t}), \forall t>0$.

\section{Prova}

O k-ésimo termo do D-espectro condicional $\mathbf{p}(\mathrm{t})$, no instante t, é $p_{k}(t)=\frac{f_{k} \cdot P\left(R_{k}>t\right)}{\sum_{j=1}^{n} f_{j} \cdot P\left(R_{j}>t\right)}$. 
Tomando-se o limite quando t tende a zero temos:

$$
p_{k}(t)=\frac{f_{k} \cdot P\left(R_{k}>t\right)}{\sum_{j=1}^{n} f_{j} \cdot P\left(R_{j}>t\right)} \rightarrow_{t \rightarrow 0} \frac{f_{k} \cdot P\left(R_{k}>0\right)}{\sum_{j=1}^{n} f_{j} \cdot P\left(R_{j}>0\right)}=f_{k} .
$$

Assim

$$
\sum_{j=k+1}^{n} p_{j}(t) \rightarrow_{t \rightarrow 0} \sum_{j=k+1}^{n} f_{j}=\bar{F}_{k}
$$

Parte (a) Provemos a suficiência do resultado.

Por hipótese $\mathbf{p}_{\mathbf{1}}(\mathbf{t}) \leq_{S T} \mathbf{p}_{\mathbf{2}}(\mathbf{t})$. Então, para todo $\mathbf{j}=1,2, \ldots \mathrm{n}$

$$
\sum_{k=j+1}^{n}\left[p_{2 k}(t)-p_{1 k}(t)\right] \geq 0 \Longleftrightarrow \sum_{k=j+1}^{n} \frac{f_{2 k} \cdot P\left(R_{k}>t\right)}{P\left(T_{2}>t\right)}-\frac{f_{1 k} \cdot P\left(R_{k}>t\right)}{P\left(T_{1}>t\right)} \geq 0,
$$

onde $R_{k}$ é o instante da k-ésima falha dos links do network, $\mathrm{k}=1, \ldots, \mathrm{n}$.

Tomando o limite quando t tende a zero, temos

$$
\sum_{k=j+1}^{n}\left[f_{2 k}-f_{1 k}\right] \geq 0
$$

A última expressão vale pois $P\left(R_{k}>0\right)=1$ e $P\left(T_{i}>0\right)=1$, para $\mathrm{i}=1,2$.

Conclui-se que, para todo $\mathrm{j}=1,2, \ldots, \mathrm{n} \bar{F}_{2 j}-\bar{F}_{1 j} \geq 0$ e portanto, $\mathbf{f}_{1} \leq_{S T} \mathbf{f}_{2}$, pela Definição A.2.1.

Para provar a condição necessária, basta supor, por absurdo, que $\mathbf{p}_{1}(t) \not_{S T} \mathbf{p}_{2}(t)$, ou seja, existe $j_{0}=1,2, \ldots, \mathrm{n}$ tal que

$$
\sum_{k=j_{0}+1}^{n}\left[p_{2 k}(t)-p_{1 k}(t)\right]<0 .
$$

Tomando-se o limite quando t tende a zero como anteriormente, resulta que, existe $j_{0}=1,2, \ldots, \mathrm{n}$ tal que

$$
\bar{F}_{2 j_{0}}-\bar{F}_{1 j_{0}}<0
$$

isto é, $\mathbf{f}_{\mathbf{1}} \bigsqcup_{S T} \mathbf{f}_{\mathbf{2}}$, o que é uma contradição, pois, por hipótese, $\mathbf{f}_{\mathbf{1}} \leq_{S T} \mathbf{f}_{\mathbf{2}}$., sob a Definição A.2.1.

Parte (b) Provemos a condição suficiente.

Suponha $\mathbf{p}_{\mathbf{1}}(\mathbf{t}) \leq_{H R} \mathbf{p}_{\mathbf{2}}(\mathbf{t})$. Então, para todo $\mathrm{k}=1,2, \ldots, \mathrm{n}, \mathrm{j}=1,2, \ldots \mathrm{n}, k \geq j$ e t $>0$ fixado,

$$
\frac{\sum_{l=k+1}^{n} p_{2 l}(t)}{\sum_{l=k+1}^{n} p_{1 l}(t)} \geq \frac{\sum_{m=j+1}^{n} p_{2 m}(t)}{\sum_{m=j+1}^{n} p_{1 m}(t)}
$$

equivalentementemente

$$
\sum_{l=k+1}^{n} p_{2 l}(t) \sum_{m=j+1}^{n} p_{1 m}(t)-\sum_{l=k+1}^{n} p_{1 l}(t) \sum_{m=j+1}^{n} p_{2 m}(t) \geq 0 .
$$

Tomando o limite quando t tende a zero de forma análoga à parte (a) do teorema, temos:

$$
\sum_{l=k+1}^{n} f_{2 l} \sum_{m=j+1}^{n} f_{1 m}-\sum_{l=k+1}^{n} f_{1 l} \sum_{m=j+1}^{n} f_{2 m} \geq 0 .
$$


Concluímos que, para todo $\mathbf{j}=1,2, \ldots, \mathrm{n} \bar{F}_{2 k} \bar{F}_{1 j}-\bar{F}_{2 j} \cdot \bar{F}_{1 k} \geq 0$ e, portanto, $\mathbf{f}_{\mathbf{1}} \leq_{H R} \mathbf{f}_{\mathbf{2}}$, pela Definição A.2.2.

Para provar a condição necessária da parte (b), suponha, por absurdo, que $\mathbf{p}_{\mathbf{1}}(\mathbf{t}) \not_{H R} \mathbf{p}_{\mathbf{2}}(\mathbf{t})$, ou seja, existem $k_{0}=1,2, \ldots, \mathrm{n}$ e $j_{0}=1,2, \ldots, \mathrm{n}$ tais que

$$
\sum_{l=k_{0}+1}^{n} p_{2 l}(t) \sum_{m=j_{0}+1}^{n} p_{1 m}(t)-\sum_{l=k_{0}+1}^{n} p_{1 l}(t) \sum_{m=j_{0}+1}^{n} p_{2 m}(t)<0 .
$$

Quando t tende a zero, concluímos que existem $k_{0}=1,2, \ldots, \mathrm{n}$ e $j_{0}=1,2, \ldots, \mathrm{n}$, tais que

$$
\bar{F}_{2 k_{0}} \bar{F}_{1 j_{0}}-\bar{F}_{2 j_{0}} \cdot \bar{F}_{1 k_{0}}<0
$$

e, portanto, $\mathbf{f}_{\mathbf{1}} \not_{H R} \mathbf{f}_{\mathbf{2}}$, o que é uma contradição à hipótese $\mathbf{f}_{\mathbf{1}} \leq_{H R} \mathbf{f}_{\mathbf{2}}$, sob a Definição A.2.2.

\section{Parte (c)}

O resultado é suficiente pois, por hipótese, $\mathbf{p}_{\mathbf{1}}(\mathbf{t}) \leq_{L R} \mathbf{p}_{\mathbf{2}}(\mathbf{t})$, isto é, para todos $\mathrm{k}, \mathrm{j}=1, \ldots, \mathrm{n}, k \geq j$ e $t>0$ fixado

$$
p_{2 k}(t) \cdot p_{1 j}(t)-p_{2 k}(t) \cdot p_{1 j}(t) \geq 0
$$

Quando t tende a zero, para todo $k, j=1, \ldots, n$, temos

$$
f_{2 k} \cdot f_{1 j}-f_{2 k} \cdot f_{1 j} \geq 0
$$

Portanto, $\mathbf{f}_{1} \leq_{L R} \mathbf{f}_{2}$, pela Definição A.2.3.

Prova-se a recíproca supondo, por absurdo, que $\mathbf{p}_{\mathbf{1}}(\mathbf{t}) \not_{L R} \mathbf{p}_{\mathbf{2}}(\mathbf{t})$, ou seja, existem $k_{0}=1,2, \ldots, n$ e $j_{0}=1,2, \ldots, n$ tais que

$$
p_{2 k_{0}}(t) \cdot p_{1 j_{0}}(t)-p_{2 k_{0}}(t) \cdot p_{1 j_{0}}(t)<0 \text {. }
$$

Quando t tende a zero, concluímos que existem $k_{0}=1,2, \ldots, n$ e $j_{0}=1,2, \ldots, n$ tais que

$$
f_{2 k_{0}} \cdot f_{1 j_{0}}-f_{2 k_{0}} . f_{1 j_{0}}<0
$$

e portanto $\mathbf{f}_{1} \leq_{L R} \mathbf{f}_{2}$, contradizendo a hipótese $\mathbf{f}_{1} \leq_{L R} \mathbf{f}_{2}$, pela Definição A.2.3.

Concluímos que se o D-espectro de um network é mais confiável, o D-espectro condicional permanece mais confiável na dinâmica do tempo. Contudo, se o D-espectro do network está mais propenso a falhas, o respectivo D-espectro condicional é mais propenso a falhas e, portanto, menos confiável no decorrer do tempo.

\subsection{Preservações das desigualdades estocásticas entre D-espectro e tempo de vida de networks}

Enunciamos um exemplo de preservação das desigualdades estocásticas entre os D-espectros de networks para seus respectivos tempos de vidas. Este exemplo foi produzido e verificado pelo próprio autor. 
Exemplo 2.3.1 (verificado no Exemplo A.4.2) Considere dois networks com 3 links cada. Sejam $\mathbf{f}_{1}=(0,5 ; 0,2 ; 0,3)$ e $\mathbf{f}_{2}=(0,4 ; 0,2 ; 0,4)$ os D-espectros dos networks. Considere $T_{1}$ e $T_{2}$ seus respectivos tempos de vidas e que suas falhas ocorrem segundo um Processo de Poisson Homogêneo com taxa $\lambda=2$ falhas/hora.

Pretende-se estudar a preservação das desigualdades estocásticas para os networks.

Sabemos, pelo Exemplo 2.2.1, que $\mathbf{f}_{\mathbf{1}} \leq_{S T} \mathbf{f}_{\mathbf{2}}, \mathbf{f}_{\mathbf{1}} \leq_{H R} \mathbf{f}_{\mathbf{2}}$ e $\mathbf{f}_{\mathbf{1}} \leq_{L R} \mathbf{f}_{\mathbf{2}}$.

Para analisar a preservação da ordenação estocástica dos networks, temos:

$$
\begin{gathered}
P\left(T_{1}>t\right)=e^{-2 t}\left(1+t+0,6 \cdot t^{2}\right) ; \\
P\left(T_{2}>t\right)=e^{-2 t}\left(1+1,2 . t+0,8 . t^{2}\right) .
\end{gathered}
$$

Concluímos que $P\left(T_{1}>t\right) \leq P\left(T_{2}>t\right)$ e $T_{1} \leq_{S T} T_{2}$, pela Definição A.2.1.

Para estudar a preservação da desigualdade por taxas de falhas dos networks, temos

$$
\frac{P\left(T_{2}>t\right)}{P\left(T_{1}>t\right)}=\frac{1+1,2 . t+0,8 . t^{2}}{1+t+0,6 . t^{2}}
$$

Assim

$$
\frac{d}{d t} \frac{P\left(T_{2}>t\right)}{P\left(T_{1}>t\right)}=\frac{0,2+0,4 \cdot t+0,08 \cdot t^{2}}{\left(1+t+0,6 . t^{2}\right)^{2}} \geq 0
$$

Concluímos que $\frac{P\left(T_{2}>t\right)}{P\left(T_{1}>t\right)}$ é crescente em t $>0$ e, portanto, $T_{1} \leq_{H R} T_{2}$, pela Definição A.2.2.

Analisaremos a preservação da desigualdade através da razão de verossimilhanças. Seja

$$
h_{i}(t)=\sum_{k=1}^{3} f_{i k} . g_{k}(t),
$$

a função densidade de probabilidade do tempo de vida do i-ésimo network no instante t $>0, \mathrm{i}=1,2$. $g_{k}(t)$ é a função densidade de probabilidade de $R_{k}, \mathrm{k}=1,2 \ldots$.

Assim

$$
\begin{gathered}
h_{1}(t)=e^{-2 t} \cdot\left(1+0,8 . t+1,2 . t^{2}\right), \\
h_{2}(t)=e^{-2 t} \cdot\left(0,8+0,8 . t+1,6 . t^{2}\right) .
\end{gathered}
$$

Portanto

$$
\begin{gathered}
\frac{h_{2}(t)}{h_{1}(t)}=\frac{0,8+0,8 . t+1,6 . t^{2}}{1+0,8 . t+1,2 . t^{2}} \\
\frac{d}{d t} \frac{h_{2}(t)}{h_{1}(t)}=\frac{0,16+1,28 . t+0,32 . t^{2}}{\left(1+0,8 . t+1,2 . t^{2}\right)^{2}} \geq 0 .
\end{gathered}
$$

Concluímos que $\frac{h_{2}(t)}{h_{1}(t)}$ é crescente em t $>0$ e, portanto, $T_{1} \leq_{L R} T_{2}$, pela Definição A.2.3.

Do exemplo concluímos que as desigualdades estocásticas dos D-espectros dos networks foram preservadas em seus tempos de vidas. Este fato é sempre verdadeiro e apresentamos o teorema de preservação das desigualdades estocásticas entre D-espectros para os respectivos networks. A 
motivação para a prova deste teorema é verificar que a comparação estocástica entre os D-espectros de networks é suficiente para comparar os tempos de vidas dos networks.

Teorema 2.3.1. (vide Teorema 3.7 de Zarezadeh e Asadi (2013)) Sejam $f_{1}$ e $f_{2}$ Despectros de dois networks cujas falhas ocorrem segundo um mesmo Processo de Poisson Não Homogêneo com função de risco $\Lambda(t), \mathrm{t}>0$. Sejam $T_{1}$ e $T_{2}$ os respectivos tempos de vida. Então:

(i) Se $\mathbf{f}_{1} \leq_{S T} \mathbf{f}_{2}$, então $T_{1} \leq_{S T} T_{2}$;

(ii) Se $\mathbf{f}_{1} \leq_{H R} \mathbf{f}_{2}$, então $T_{1} \leq_{H R} T_{2}$;

(iii) Se $\mathbf{f}_{1} \leq_{L R} \mathbf{f}_{2}$, então $T_{1} \leq_{L R} T_{2}$.

\section{Prova}

É desenvolvida pelo próprio autor da dissertação.

Para provar a parte (i) mostraremos que, para todo $\mathrm{t}>0 \bar{H}_{2}(t) \geq \bar{H}_{1}(t)$, onde $\bar{H}_{i}(t)=$ $E\left[\bar{F}_{i, N(t)}\right]=\sum_{k=0}^{n-1} \bar{F}_{i k} \cdot \frac{e^{-\Lambda(t)} . \Lambda(t)^{k}}{k !}$ é a confiabilidade do i-ésimo network no instante t $>0, \mathrm{i}=1,2$.

Como por hipótese $\mathbf{f}_{1} \leq_{S T} \mathbf{f}_{2}$, isto é, $\bar{F}_{1 k} \leq \bar{F}_{2 k}, \forall k=0, \ldots n-1$ (Definição A.2.1), temos

$$
\bar{H}_{2}(t)=E\left[\bar{F}_{2, N(t)}\right] \geq E\left[\bar{F}_{1, N(t)}\right]=\bar{H}_{1}(t),
$$

e, portanto, $T_{1} \leq_{S T} T_{2}$ (Definição A.2.1).

Para provar a parte (ii) verificaremos que $\bar{H}_{i}(t)$ é $T P_{2}$ em i e t, com i=1,2 e t $>0$.

Por hipótese, $\mathbf{f}_{1} \leq_{H R} \mathbf{f}_{2}$, isto é, sob a Definição A.2.2, se $\mathrm{k} \geq \mathrm{j}=0, \ldots, \mathrm{n}-1$

$$
\bar{F}_{2 k} \cdot \bar{F}_{1 j}-\bar{F}_{2 j} \cdot \bar{F}_{1 k} \geq 0
$$

e se $\mathrm{k}<\mathrm{j}=0, \ldots, \mathrm{n}-1$

$$
\bar{F}_{2 k} \cdot \bar{F}_{1 j}-\bar{F}_{2 j} \cdot \bar{F}_{1 k}<0 .
$$

Do Exemplo A.2.2 sabemos que $\mathrm{P}(\mathrm{N}(\mathrm{t})=\mathrm{k})$ é $\mathrm{TP}_{2}$ em $\mathrm{i}=0, \ldots, \mathrm{n}-1$ e $\mathrm{t}>0$, ou seja, para $0 \leq s \leq t$, se $\mathrm{k} \geq \mathrm{j}$

$$
P(N(t)=k) \cdot P(N(s)=j)-P(N(t)=j) \cdot P(N(s)=k) \geq 0,
$$

e se $\mathrm{k}<\mathrm{j}$

$$
P(N(t)=k) \cdot P(N(s)=j)-P(N(t)=j) \cdot P(N(s)=k)<0 .
$$

Portanto

$$
\left(\bar{F}_{2 k} \cdot \bar{F}_{1 j}-\bar{F}_{2 j} \cdot \bar{F}_{1 k}\right) \cdot(P(N(t)=k) \cdot P(N(s)=j)-P(N(t)=j) \cdot P(N(s)=k)) \geq 0 .
$$

A desigualdade resulta do produto de expressões de mesmo sinal. Assim

$$
\begin{gathered}
\sum_{k=0}^{n-1} \sum_{j=0}^{n-1}\left(\bar{F}_{2 k} \cdot \bar{F}_{1 j}-\bar{F}_{2 j} \cdot \bar{F}_{1 k}\right) \cdot(P(N(t)=k) \cdot P(N(s)=j)-P(N(t)=j) \cdot P(N(s)=k)) \geq 0 \\
\Longleftrightarrow \sum_{k=0}^{n-1} \bar{F}_{2 k} \cdot\left(P(N(t)=k) \sum_{j=0}^{n-1} \bar{F}_{1 j} \cdot P(N(s)=j)-\sum_{k=0}^{n-1} \bar{F}_{1 k}\right) \cdot\left(P(N(s)=k) \cdot \sum_{j=0}^{n-1} \bar{F}_{2 j} \cdot P(N(t)=j)\right.
\end{gathered}
$$




$$
-\sum_{k=0}^{n-1} \bar{F}_{1 k} \cdot\left(P(N(t)=k) \sum_{j=0}^{n-1} \bar{F}_{2 j} \cdot P(N(s)=j)+\sum_{k=0}^{n-1} \bar{F}_{1 k}\right) \cdot\left(P(N(s)=k) \cdot \sum_{j=0}^{n-1} \bar{F}_{2 j} \cdot P(N(t)=j) \geq 0,\right.
$$

e concluímos que

$$
P\left(T_{2}>t\right) \cdot P\left(T_{1}>s\right)-P\left(T_{2}>s\right) \cdot P\left(T_{1}>t\right) \geq 0 .
$$

Então $P\left(T_{i}>t\right)$ é $\mathrm{TP}_{2}$ em i=1,2 e t $>0$ e, portanto, $T_{1} \leq_{H R} T_{2}$, sob a Definição A.2.2.

Para provar a parte (iii) basta verificar que, para $0 \leq s \leq t$

$$
h_{2}(t) \cdot h_{1}(s)-h_{2}(s) \cdot h_{1}(t) \geq 0,
$$

onde $h_{i}(t)=\sum_{k=1}^{n} f_{i k} . g_{k}(t)$ é a função densidade de probabilidade do tempo de vida i-ésimo network no instante $\mathrm{t}>0, \mathrm{i}=1,2$, e $g_{k}(t)$ é a função densidade de probabilidade de $R_{k}, \mathrm{k}=1,2, \ldots \mathrm{n}$.

Para $0 \leq s \leq t, 0 \leq j \leq k, \mathrm{j}, \mathrm{k}=1, \ldots, \mathrm{n}$, temos

$$
\frac{g_{k}(t)}{g_{j}(t)}=\frac{\lambda(t) \cdot P(N(t)=k-1)}{\lambda(t) \cdot P(N(t)=j-1)} \geq \frac{P(N(s)=k-1)}{P(N(s)=j-1)}=\frac{\lambda(s) \cdot P(N(s)=k-1)}{\lambda(s) \cdot P(N(s)=j-1)}=\frac{g_{k}(s)}{g_{j}(s)},
$$

onde $\lambda(t)$ é a taxa de falha do instante da primeira falha dos links. A desigualdade vale pois $\mathrm{P}(\mathrm{N}(\mathrm{t})=\mathrm{k})$ é $\mathrm{TP}_{2}$ em $\mathrm{k}=1, . ., \mathrm{n}$ e $\mathrm{t}>0$. Portanto $g_{k}(t)$ é $\mathrm{TP}_{2}$ em $\mathrm{k}=1,2, . ., \mathrm{n}$ e $\mathrm{t}>0$.

A prova ocorre de maneira análoga à parte (ii) do teorema, substituindo-se $\bar{F}_{i k}$ e $\mathrm{P}(\mathrm{N}(\mathrm{t})=\mathrm{k})$ por $f_{i k}$ e $g_{k}$, respectivamente, $\mathrm{i}=1,2, \mathrm{k}=1,2, \ldots \mathrm{n}$.

Concluímos que $h_{2}(t) \cdot h_{1}(s)-h_{2}(s) \cdot h_{1}(t) \geq 0$ e, portanto, $T_{1} \leq_{L R} T_{2}$, sob a Definição A.2.3.

Observação 2.2.1 Em relação à preservação da desigualdade estocástica usual entre networks, concluímos que um network que tenha probabilidade maior de sobreviver uma quantidade de falhas de links seja mais confiável.

Entendemos que se um D-espectro é mais propenso a falhas, então seu respectivo network é menos confiável.

A desigualdade por razão de verossimilhanças implica as desigualdades estocásticas e as ordenações estocásticas (Teorema A.2.1).

No que segue provaremos resultados de equivalência de desigualdades estocásticas entre Despectros e D-espectros condicionais de networks cujas falhas dos links ocorrem segundo um Processo de Poisson Não Homogêneo.

Analisaremos a preservação das desigualdades estocásticas entre os D-espectros condicionais e os tempos de vida residuais de dois networks.

\subsection{Resultados de Preservações entre D-espectros condicionais e tempos de vidas residuais}

Veremos um exemplo da preservação das desigualdades estocásticas de D-espectros condicionais para tempos de vidas residuais de networks. Este exemplo é produção do autor deste trabalho.

Exemplo 2.4.1 (ver Exemplo A.4.3.) Considere dois networks com D-espectros $\mathbf{f}_{1}=(0,4$; $0,4 ; 0,2)$ e $\mathbf{f}_{2}=(0,2 ; 0,4 ; 0,4)$ e $\mathbf{p}_{1}(t)$ e $\mathbf{p}_{2}(t)$ os respectivos D-espectros condicionais no instante $\mathrm{t}$ 
$>0$. Sejam $T_{1}$ e $T_{2}$ seus tempos de vidas e $\left\{T_{1}-t \mid T_{1}>t\right\}$ e $\left\{T_{2}-t \mid T_{2}>t\right\}$ os respectivos tempos de vidas residuais no instante t. Suponha que as falhas dos links dos networks ocorrem segundo um mesmo Processo de Poisson Homogêneo com taxa $\lambda=1$ falha/hora.

A confiabilidade do i-ésimo network é

$$
P\left(T_{i}>t\right)=\sum_{k=0}^{2} \bar{F}_{k} \cdot P(N(t)=k),
$$

onde $\bar{F}_{i k}$ é a função de sobrevivência do D-espectro do i-ésimo network a k falhas de seus links, $\mathrm{i}=1,2, \mathrm{k}=0,1,2 \ldots \mathrm{n}-1$ (Zarezadeh e Asadi, 2013).

Temos: $\bar{F}_{11}=\bar{F}_{21}=1, \bar{F}_{12}=0,6, \bar{F}_{22}=0,8, \bar{F}_{13}=0,2, \bar{F}_{23}=0,4$.

O k-ésimo componente do D-espectro condicional do i-ésimo network no instante t é

$$
p_{i k}(t)=\frac{f_{i k} \cdot P\left(R_{k}>t\right)}{P\left(T_{i}>t\right)}
$$

onde $R_{k}$ é o instante da k-ésima falha dos links (ver Definição 2.1.1).

Assim

$$
\begin{gathered}
P\left(R_{1}>t\right)=e^{-t} \\
P\left(R_{2}>t\right)=e^{-t}(1+t) \\
P\left(R_{3}>t\right)=e^{-t}\left(1+t+\frac{t^{2}}{2}\right) .
\end{gathered}
$$

$\mathrm{E}$

$$
\begin{aligned}
& P\left(T_{1}>t\right)=e^{-t}\left(1+0,6 t+0,1 t^{2}\right) \\
& P\left(T_{2}>t\right)=e^{-t}\left(1+0,8 t+0,2 t^{2}\right) .
\end{aligned}
$$

As funções densidades de probabilidade dos tempos de vidas dos networks são (ver Zarezadeh e Asadi (2013))

$$
\begin{aligned}
& h_{1}(t)=0,2 \cdot e^{-t} \cdot(2+t)^{2} ; \\
& h_{2}(t)=0,2 \cdot e^{-t} \cdot(1+t)^{2} .
\end{aligned}
$$

Verificaremos que $\mathbf{p}_{\mathbf{1}}(\mathbf{t}) \leq_{L R} \mathbf{p}_{\mathbf{2}}(\mathbf{t})$, isto é, $\frac{p_{2 k}(t)}{p_{1 k}(t)}$ é crescente em k (Definição A.2.3). Temos:

$$
\begin{gathered}
p_{11}(t)=\frac{0,4}{1+0,6 t+0,1 t^{2}} \\
p_{12}(t)=\frac{(0,4+0,4 t)}{\left(1+0,6 t+0,1 t^{2}\right)} \\
p_{13}(t)=\frac{0,2+0,2 t+0,1 . t^{2}}{1+0,6 t+0,1 t^{2}} \\
p_{21}(t)=\frac{0,2}{1+0,8 t+0,2 t^{2}}
\end{gathered}
$$




$$
\begin{gathered}
p_{22}(t)=\frac{(0,4+0,4 t)}{\left(1+0,8 t+0,2 t^{2}\right)} \\
p_{23}(t)=\frac{0,4+0,4 t+0,2 \cdot t^{2}}{1+0,8 t+0,2 t^{2}} .
\end{gathered}
$$

Portanto

$$
\begin{aligned}
& \frac{p_{21}(t)}{p_{11}(t)}=\frac{1}{2} \cdot\left(\frac{1+0,6 t+0,1 t^{2}}{1+0,8 t+0,2 t^{2}}\right) ; \\
& \frac{p_{22}(t)}{p_{12}(t)}=1 \cdot\left(\frac{1+0,6 t+0,1 t^{2}}{1+0,8 t+0,2 t^{2}}\right) ; \\
& \frac{p_{23}(t)}{p_{13}(t)}=2 \cdot\left(\frac{1+0,6 t+0,1 t^{2}}{1+0,8 t+0,2 t^{2}}\right) .
\end{aligned}
$$

Concluímos que $\frac{p_{21}(t)}{p_{11}(t)} \leq \frac{p_{22}(t)}{p_{12}(t)} \leq \frac{p_{23}(t)}{p_{13}(t)}$ e $\mathbf{p}_{\mathbf{1}}(\mathbf{t}) \leq_{L R} \mathbf{p}_{\mathbf{2}}(\mathbf{t})$.

Pelo Teorema A.2.1 isto implica $\mathbf{p}_{\mathbf{1}}(\mathbf{t}) \leq_{H R} \mathbf{p}_{\mathbf{2}}(\mathbf{t})$, consequentemente $\mathbf{p}_{\mathbf{1}}(\mathbf{t}) \leq_{S T} \mathbf{p}_{\mathbf{2}}(\mathbf{t})$.

A função densidade do tempo de vida residual do i-ésimo network em $\mathrm{x}$, dado $\left\{T_{i}>t\right\}$ é

$$
h_{i}(x \mid t)=\frac{h_{i}(x+t)}{P\left(T_{i}>t\right)} .
$$

Verificaremos que $\left\{T_{1}-t \mid T_{1}>t\right\} \leq_{L R}\left\{T_{2}-t \mid T_{2}>t\right\}$, isto é $\frac{h_{2}(x \mid t)}{h_{1}(x \mid t)}$ é crescente em x (Definição A.2.3).

Observe que

$$
\frac{h_{2}(x \mid t)}{h_{1}(x \mid t)}=\frac{\frac{h_{2}(x+t)}{P\left(T_{2}>t\right)}}{\frac{h_{1}(x+t)}{P\left(T_{1}>t\right)}}=\frac{P\left(T_{1}>t\right)}{P\left(T_{2}>t\right)} \frac{h_{2}(t+x)}{h_{1}(t+x)} .
$$

Como $\frac{P\left(T_{1}>t\right)}{P\left(T_{2}>t\right)}$ não depende de x e é positivo, basta verificar que $\frac{h_{2}(t+x)}{h_{1}(t+x)}$ é crescente em $\mathrm{x}$. Portanto

$$
\frac{d}{d x} \frac{h_{2}(t+x)}{h_{1}(t+x)}=\frac{2(1+t+x)}{(2+t+x)^{3}} \geq 0
$$

Concluímos que $\frac{h_{2}(x \mid t)}{h_{1}(x \mid t)}$ é crescente em x e $\left\{T_{1}-t \mid T_{1}>t\right\} \leq_{L R}\left\{T_{2}-t \mid T_{2}>t\right\}$. Pelo Teorema A.2.1, isto implica $\left\{T_{1}-t \mid T_{1}>t\right\} \leq_{H R}\left\{T_{2}-t \mid T_{2}>t\right\}$ e consequentemente $\left\{T_{1}-t \mid T_{1}>t\right\} \leq_{S T}$ $\left\{T_{2}-t \mid T_{2}>t\right\}$.

Enunciaremos um lema que auxiliará na prova da preservação de desigualdades estocásticas entre os D-espectros condicionais e os tempos de vida residuais de networks. O lema é desenvolvido e provado pelo próprio autor.

Lema 2.4.1 Seja $R_{k}$ o instante da k-ésima falha dos links de um network, que ocorrem segundo um Processo de Poisson Não Homogêneo com função de risco $\Lambda(t), t>0$. Seja $g_{k}(t)$ a respectiva função densidade de probabilidade no instante $\mathrm{t}>0, \mathrm{k}=1,2, \ldots \mathrm{n}$. Então 
(i) $R_{k} \leq_{L R} R_{k+1}, \mathrm{k}=1,2, \ldots, \mathrm{n}$;

(ii) $\left\{R_{k}-t \mid R_{k}>t\right\} \leq_{L R}\left\{R_{k+1}-t \mid R_{k+1}>t\right\}, \mathrm{k}=1,2, \ldots, \mathrm{n}$.

\section{Prova}

Para provar a parte (i), se $0 \leq s \leq t$, temos que

$$
\frac{g_{k+1}(t)}{g_{k}(t)}=\frac{\lambda(t) \cdot \frac{e^{-\Lambda(t)} \cdot(\Lambda(t))^{k}}{(k) !}}{\frac{\lambda(t) \cdot e^{-\Lambda(t)} \cdot(\Lambda(t))^{k-1}}{(k-1) !}}=\frac{\Lambda(t)}{k},
$$

onde $\lambda(t)$ é a taxa de falha do instante da primeira ocorrência do processo e $\lambda(t)=\frac{d \Lambda(t)}{d t}$

Como $\Lambda(t)$ é crescente em $\mathrm{t}, \frac{g_{k+1}(t)}{g_{k}(t)}$ é crescente em $\mathrm{t}>0$ e, portanto, $R_{k} \leq_{L R} R_{k+1}$ (ver Definição A.2.3).

Para provar a parte (ii), seja $g_{k}(x \mid t)$ a função densidade de probabilidade de $\left\{R_{k}-t \mid R_{k}>t\right\}$, em $\mathrm{x}>0$, para $\mathrm{t}>0$ fixado e $\mathrm{k}=1, \ldots \mathrm{n}$.

Queremos verificar que $\frac{g_{k+1}(x \mid t)}{g_{k}(x \mid t)}$ é crescente em $\mathrm{x}>0$, para $\mathrm{t}>0$ fixado. Temos:

$$
g_{k}(x \mid t)=-\frac{d}{d x} P\left(R_{k}>t+x \mid R_{k}>t\right)=-\frac{d}{d x} \frac{P\left(R_{k}>t+x\right)}{P\left(R_{k}>t\right)}=\frac{g_{k}(t+x)}{P\left(R_{k}>t\right)},
$$

e

$$
\frac{g_{k+1}(x \mid t)}{g_{k}(x \mid t)}=\frac{\frac{g_{k+1}(t+x)}{P\left(R_{k+1}>t\right)}}{\frac{g_{k}(t+x)}{P\left(R_{k}>t\right)}}=\frac{P\left(R_{k}>t\right)}{P\left(R_{k+1}>t\right)} \cdot \frac{g_{k+1}(x+t)}{g_{k}(x+t)} .
$$

Como $\frac{P\left(R_{k}>t\right)}{P\left(R_{k+1}>t\right)} \geq 0$ e $\frac{g_{k+1}(x+t)}{g_{k}(x+t)}$ é crescente em $\mathrm{x}>0$, concluímos que $\frac{g_{k+1}(x \mid t)}{g_{k}(x \mid t)}$ também o é e, portanto, $\left\{R_{k}-t \mid R_{k}>t\right\} \leq_{L R}\left\{R_{k+1}-t \mid R_{k+1}>t\right\}$ (ver Definição A.2.3).

Interessa-nos verificar que a comparação estocástica entre D-espectros condicionais é suficiente para esta comparação entre os respectivos networks na dinâmica do tempo. Tal fato é verdadeiro e é provado no teorema de preservação de desiguades estocásticas entre D-espectros condicionais de seus networks para seus respectivos tempos de vidas residuais.

Teorema 2.4.1 (ver Teorema 2.6 de Zarezadeh et al. (2013)) Sejam $T_{1}$ e $T_{2}$ os tempos de vida de dois networks com n componentes cada, cujas falhas ocorrem segundo um mesmo Processo de Poisson Não Homogêneo com função de risco $\Lambda(t), \mathrm{t}>0$. Sejam $\mathbf{f}_{\mathbf{1}}$ e $\mathbf{f}_{\mathbf{2}}$ seus D-espectros e $\mathbf{p}_{\mathbf{1}}(\mathbf{t})$ e $\mathbf{p}_{\mathbf{2}}(\mathbf{t})$ os respectivos D-espectros condicionais no instante $\mathrm{t}>0$.

(i) Se $\mathbf{p}_{\mathbf{1}}(\mathbf{t}) \leq \mathbf{S T} \mathbf{p}_{\mathbf{2}}(\mathbf{t})$, então $\left\{T_{1}-t \mid T_{1}>t\right\} \leq_{S T}\left\{T_{2}-t \mid T_{2}>t\right\}$;

(ii) Se $\mathbf{p}_{\mathbf{1}}(\mathbf{t}) \leq_{\text {HR }} \mathbf{p}_{\mathbf{2}}(\mathbf{t})$, então $\left\{T_{1}-t \mid T_{1}>t\right\} \leq_{H R}\left\{T_{2}-t \mid T_{2}>t\right\}$;

(iii) Se $\mathbf{p}_{\mathbf{1}}(\mathbf{t}) \leq_{\mathbf{L R}} \mathbf{p}_{\mathbf{2}}(\mathbf{t})$, então $\left\{T_{1}-t \mid T_{1}>t\right\} \leq_{L R}\left\{T_{2}-t \mid T_{2}>t\right\}$.

\section{Prova}

(i) É suficiente verificar que, para todo $\mathrm{x}>0, P\left(T_{2}-t>x \mid T_{2}>t\right) \geq P\left(T_{1}-t>x \mid T_{1}>t\right)$

Observe que, pelo Lema 2.4.1, $\left\{R_{k}-t \mid R_{k}>t\right\} \leq_{L R}\left\{R_{k+1}-t \mid R_{k+1}>t\right\}$, consequentemente $\left\{R_{k}-t \mid R_{k}>t\right\} \leq_{H R}\left\{R_{k+1}-t \mid R_{k+1}>t\right\}$, o que implica, $\left\{R_{k}-t \mid R_{k}>t\right\} \leq_{S T}\left\{R_{k+1}-t \mid R_{k+1}>t\right\}$, usando o Teorema A.2.1.

Assim

$$
P\left(T_{2}-t>x \mid T_{2}>t\right)=\sum_{k=1}^{n} p_{2 k}(t) . P\left(R_{k}>t+x \mid R_{k}>t\right)
$$




$$
\geq \sum_{k=1}^{n} p_{1 k}(t) \cdot P\left(R_{k}>t+x \mid R_{k}>t\right)=P\left(T_{2}-t>x \mid T_{2}>t\right) .
$$

A desigualdade é válida, pois, por hipótese, $\mathbf{p}_{\mathbf{1}}(\mathbf{t}) \leq_{S T} \mathbf{p}_{\mathbf{2}}(\mathbf{t}), \mathrm{t}>0$, e $\left\{R_{k}-t \mid R_{k}>t\right\} \leq_{S T}$ $\left\{R_{k+1}-t \mid R_{k+1}>t\right\}, \mathrm{k}=1,2 \ldots \mathrm{n}$.

Portanto, usando o Teorema A.2.2 (a) e a Definição A.2.1, $\left\{T_{1}-t \mid T_{1}>t\right\} \leq_{S T}\left\{T_{2}-t \mid T_{2}>t\right\}$.

(ii) Verificaremos que $P\left(T_{i}>t+x \mid T_{i}>t\right)$ é $\mathrm{TP}_{2}$ em $\mathrm{i}=1,2 \mathrm{e} \mathrm{x}>0$, para $\mathrm{t}>0$ fixado.

Sabemos que $\left\{R_{k}-t \mid R_{k}>t\right\} \leq_{L R}\left\{R_{k+1}-t \mid R_{k+1}>t\right\}$. Pelo Teorema A.2.1, temos $\left\{R_{k}-t \mid R_{k}>\right.$ $t\} \leq_{H R}\left\{R_{k+1}-t \mid R_{k+1}>t\right\}$.

Como $\mathbf{p}_{\mathbf{1}}(\mathbf{t}) \leq_{H R} \mathbf{p}_{\mathbf{2}}(\mathbf{t})$ por hipótese, pelo Teorema A.2.2 (b) e a Definição A.2.2, concluímos que $\left\{T_{1}-t \mid T_{1}>t\right\} \leq_{H R}\left\{T_{2}-t \mid T_{2}>t\right\}$.

(iii) Considere $h_{i}(x \mid t)$ a função densidade de probabilidade de $\left\{T_{i}-t \mid T_{i}>t\right\}$ para $\mathrm{x}>0$ e t $>$ 0 fixado, tal que $h_{i}(x \mid t)=\sum_{k=1}^{n} f_{i k} . g_{k}(x \mid t)$.

Basta verificar que $h_{i}(x \mid t)$ é $\mathrm{TP}_{2}$ em $\mathrm{i}=1,2$ e $\mathrm{x}>0$, para $\mathrm{t}>0$ fixado.

Usando o Lema 2.4.1 $\left\{R_{k}-t \mid R_{k}>t\right\} \leq_{L R}\left\{R_{k+1}-t \mid R_{k+1}>t\right\}$.

Como $\mathbf{p}_{\mathbf{1}}(\mathbf{t}) \leq_{L R} \mathbf{p}_{\mathbf{2}}(\mathbf{t})$ por hipótese, pelo Teorema A.2.2 (c) e a Definição A.2.3, concluímos que $\left\{T_{1}-t \mid T_{1}>t\right\} \leq_{L R}\left\{T_{2}-t \mid T_{2}>t\right\}$.

Concluímos que um network com D-espectro condicional mais confiável permanece mais confiável na dinâmica do tempo. Contudo se o respectivo D-espectro condicional é mais propenso a falhas, então o network também o é com o decorrer do tempo e, portanto, menos confiável (Teorema A.2.1).

No próximo capítulo, analisaremos a preservação das classes de distribuições para os networks em estudo. 


\section{Capítulo 3}

\section{Resultados de preservação das classes de distribuições}

Um dos interesses do estudo de classes de distribuições úteis na Teoria da Confiabilidade é sua aplicação em testes de vida, políticas de manutenção, políticas de garantias, etc. Neste sentido tornase importante considerar tais conceitos para o D-espectro do network e analisar em que condições as propriedades são preservadas para seu tempo de vida. Parece-nos que um estudo sistemático de tais procedimentos não aparece na literatura de networks.

Neste capítulo estudaremos resultados de preservação de diversas classes de distribuições do D-espectro de um network para seu respectivo tempo de vida.

\subsection{Resultados de preservação de classes de distribuições do D- espectro para o tempo de vida de um network}

Uma classe de distribuições importante é a das distribuições com taxa de falha crescente (IFR), em que a idade é adversa, no sentido de que o tempo de vida residual decresce estocasticamente no tempo. Se a idade do objeto é benéfica, de forma que a probabilidade de sobrevivência condicional é crescente em função da idade tem taxa de falha decrescente (DFR). Em termos do D-espectro definimos tais classes de distribuições.

Definição 3.1.1 (Barlow e Proschan, 1975) Considere um network com D-espectro f. Dizemos que o D-espectro ou sua distribuição tem taxa de falha crescente (decrescente) - IFR (DFR) se, para $\mathrm{m}=1,2 \ldots \mathrm{n}$, fixado $\frac{\bar{F}_{k+m-1}}{\bar{F}_{k-1}}$ é decrescente (crescente) em $\mathrm{k}=1,2 \ldots \mathrm{n}$.

Equivalentemente, o D-espectro f é IFR (DFR) se $\frac{f_{k}}{F_{k-1}}$ é crescente (decrescente) em k=1,2..n.

Exemplo 3.1.1 (vide Exemplo A.4.4) Seja $\mathbf{f}=(0,5 ; 0,2 ; 0,3)$ o D-espectro de um network.

Considere $\bar{F}$ um vetor com a função de sobrevivência do D-espectro, da forma

$\bar{F}=\left(\bar{F}_{0}, \bar{F}_{1}, \bar{F}_{2}\right)=(1 ; 0,5 ; 0,3)$.

Verificamos que

$$
\frac{\bar{F}_{2}}{\bar{F}_{1}} \leq \frac{\bar{F}_{1}}{\bar{F}_{0}} \Longleftrightarrow \bar{F}_{1}^{2}-\bar{F}_{2} \cdot \bar{F}_{0}=0,5^{2}-1.0,3=-0,05 \leq 0
$$

e, portanto, f é DFR. 
Veremos um exemplo da preservação da classe IFR (DFR) do D-espectro para o respectivo network.

Exemplo 3.1.2 (ver Exemplo A.4.5) Considere um network com D-espectro $\mathbf{f}=(0,6 ; 0,1$; $0,3)$. Seja $\Lambda(t)=1-e^{-t}$

Verificamos a classe de distribuições do D-espectro $\mathbf{f}$. Temos que

$$
\frac{\bar{F}_{1}}{\bar{F}_{0}}-\frac{\bar{F}_{2}}{\bar{F}_{1}}=\frac{0,4}{1}-\frac{0,3}{0,4}=0,4-0,75=-0,35 \leq 0,
$$

onde $\bar{F}_{k}$ é a função de sobrevivência do D-espectro a k falhas de seus links. Então, sob a Definição 3.1.1, f é DFR.

Verificamos a convexidade da função de risco $\Lambda(t)$

$$
\begin{gathered}
\frac{d \Lambda(t)}{d t}=e^{-t} \\
\frac{d^{2} \Lambda(t)}{d t^{2}}=-e^{-t}<0 .
\end{gathered}
$$

Então a função de risco é côncava.

A confiabilidade do network é

$$
\bar{H}(t)=e^{-1} e^{-e^{-t}} \cdot\left(1,55-0,7 . e^{-t}+0,15 e^{-2 t}\right) .
$$

A função densidade de probabilidade de T é

$$
h(t)=e^{-\left(1-e^{-t}\right)}\left(0,85 e^{-t}-0,4 e^{-2 t}+0,15 e^{-3 t}\right) .
$$

A taxa de falha do network é

$$
r(t)=\frac{0,85 e^{-t}-0,4 e^{-2 t}+0,15 e^{-3 t}}{1,55-0,7 . e^{-t}+0,15 e^{-2 t}} .
$$

Verificamos que a taxa de falha é decrescente

$$
\frac{d r(t)}{d t}=-\frac{1,3175 e^{-t}+1,24 e^{-2 t}-0,85 e^{-3 t}+0,210 e^{-4 t}-0,0225 e^{-5 t}}{\left(1,55-0,7 . e^{-t}+0,15 e^{-2 t}\right)^{2}} \leq 0 .
$$

A desigualdade é válida pois: $e^{-a t} \leq 1, \forall a, t \geq 0$, e $e^{-a t} \leq e^{-b t}, a \geq b \geq 0, \forall t \geq 0$.

Sob a Definição A.3.1 e a Observação A.3.1, concluímos que T é DFR.

Analisaremos a preservação da classe de distribuição IFR (DFR) do D-espectro para o tempo de vida de um network pelo seguinte teorema. A motivação para a prova deste teorema é constatar se o estudo da classe de distribuição IFR para o D-espectro de um network e a convexidade da função de risco do Processo de Poisson Não Homogêneo para as falhas de seus links são suficientes para avaliar tal classe para o respectivo network.

Teorema 3.1.1 Seja T o tempo de vida de um network com função de distribuição contínua e D-espectro f, cujas falhas de seus links ocorrem segundo um Processo de Poisson Não Homogêneo 
com função de risco $\Lambda(t)>0, \mathrm{t}>0$. Seja $\lambda(t)$ a taxa de falha no instante da primeira falha. Se $\mathbf{f}$ é IFR (DFR), e $\Lambda(t)$, é convexa (côncava), então T é IFR (DFR).

Prova Provamos a preservação da classe de distribuições IFR.

Em primeiro lugar, considere um Processo de Poisson Homogêneo com taxa $\lambda>0$ (Teorema 3.1, parte (3.2) de Esary et al. (1973)). Neste caso, a confiabilidade do network no instante t $>0$ é

$$
\bar{H}^{*}(t)=\sum_{k=0}^{n-1} \bar{F}_{k} \cdot P(N(t)=k)=\sum_{k=0}^{n-1} \bar{F}_{k} \cdot \frac{e^{-\lambda \cdot t} \cdot(\lambda \cdot t)^{k}}{k !},
$$

onde $\bar{F}_{k}=\sum_{j=k+1}^{n} f_{j}$ é a função de sobrevivência do D-espectro em $\mathrm{k}=1,2 \ldots, \mathrm{n}$.

Como f é IFR, pela Definição 3.1.1, temos

$$
\frac{\bar{F}_{k+1}}{\bar{F}_{k}} \leq \frac{\bar{F}_{k}}{\bar{F}_{k-1}} \Longleftrightarrow \log \bar{F}_{k+1}-\log \bar{F}_{k} \leq \log \bar{F}_{k}-\log \bar{F}_{k-1} \Longleftrightarrow \log \bar{F}_{k+1}+\log \bar{F}_{k-1} \leq 2 . \log \bar{F}_{k}
$$

Então $\bar{F}_{k}$ é log-côncava em k=1,2...n.

Portanto, para $\xi>0$ e $a>0$ fixados, $\bar{F}_{k}-a . \xi^{k}$ possui no máximo duas trocas de sinais do tipo negativo-positivo-negativo. Observe que se $\bar{F}_{k}-a . \xi^{k} \leq(\geq) 0$, então $\log \bar{F}_{k} \leq(\geq) \log a+k \cdot \log \xi$, o que justifica a log-concavidade de $\bar{F}_{k}$.

Como $\mathrm{P}\left(\mathrm{N}(\mathrm{t})=\mathrm{k}\right.$ ) é $\mathrm{TP}_{2}$ em $\mathrm{k}=0 \ldots, \mathrm{n}-1$ e $\mathrm{t}>0$ (Exemplo A.2.2), usando a propriedade da diminuição da variação (ver seção A.3 do Apêndice),

$$
\begin{aligned}
\sum_{k=0}^{\infty}\left(\bar{F}_{k}-a \cdot \xi^{k}\right) \cdot \frac{e^{-\lambda \cdot t} \cdot(\lambda \cdot t)^{k}}{k !} & =\sum_{k=0}^{\infty} \bar{F}_{k} \cdot \frac{e^{-\lambda \cdot t} \cdot(\lambda \cdot t)^{k}}{k !}-\sum_{k=0}^{\infty} a \cdot \xi^{k} \cdot \frac{e^{-\lambda \cdot t} \cdot(\lambda \cdot t)^{k}}{k !}=\bar{H}(t)-a \cdot \sum_{k=0}^{\infty} e^{-\lambda \cdot t} \frac{(\xi \cdot \lambda \cdot t)^{k}}{k !} \\
& =\bar{H}^{*}(t)-a \cdot e^{-\lambda \cdot t} \cdot e^{\lambda . \xi \cdot t}=\bar{H}^{*}(t)-a \cdot e^{-(1-\xi) \cdot \lambda \cdot t}
\end{aligned}
$$

também possui no máximo duas trocas de sinais do tipo negativo-positivo-negativo em $\mathrm{t}>0$.

Considerando-se $\theta=(1-\xi) . \lambda<\lambda$, com $\xi \in(0,1)$, a última expressão tem no máximo duas trocas de sinais do tipo negativo-positivo-negativo em relação a $t>0$.

Como $\bar{H}^{*}(t)=e^{-\lambda . t}+\sum_{k=1}^{n-1} \bar{F}_{k} \cdot \frac{e^{-\lambda . t} \cdot(\lambda \cdot t)^{k}}{k !}\left(\right.$ pois $\left.\bar{F}_{0}=1\right)$, temos

$$
\bar{H}^{*}(t) \geq e^{-\lambda . t} \geq e^{-\theta \cdot t}, t>0, \text { para } \theta \geq \lambda .
$$

Então

$$
\bar{H}^{*}(t) \geq e^{-\theta \cdot t}, \forall t>0, \forall \theta>0 .
$$

Concluímos que $\bar{H}^{*}(t)-e^{-\theta . t}$ tem no máximo duas trocas de sinais do tipo negativo-positivonegativo em respeito a $\mathrm{t}>0$, portanto, $\bar{H}^{*}(t)$ é log-côncava em $\mathrm{t}>0$ e $\bar{H}^{*}(t)$ é IFR.

Considere agora a confiabilidade do network no instante $\mathrm{t}>0 \bar{H}(t)=\sum_{k=0}^{n-1} \bar{F}_{k} \cdot \frac{e^{-\Lambda(t)} . \Lambda(t)^{k}}{k !} \mathrm{e}$ sua respectiva taxa de falha $r(t)$ (Teorema 3.1 (c) de A-Hameed e Proschan (1973)).

Observe que $\bar{H}(t)$ pode ser escrito como $\bar{H}(t)=\bar{H}^{*}(\Lambda(t))$, onde

$$
\bar{H}^{*}(x)=\sum_{k=0}^{n-1} \bar{F}_{k} \cdot \frac{e^{-x} \cdot x^{k}}{k !}
$$


é a confiabilidade de um network cujas falhas dos links ocorrem segundo um Processo de Poisson Homogêneo com taxa 1, no instante $\mathrm{x}>0$, com taxa de falha $r^{*}(t)$.

Assim

$$
\frac{d}{d t} \log \bar{H}(t)=\frac{d}{d t} \log \bar{H}^{*}(\Lambda(t))=-\frac{h^{*}(\Lambda(t))}{\bar{H}^{*}(\Lambda(t))} \cdot \lambda(t)=-r^{*}(\Lambda(t)) \cdot \lambda(t) .
$$

Então

$$
\begin{gathered}
\frac{d^{2}}{d t^{2}} \log \bar{H}(t)=\frac{d^{2}}{d t^{2}} \log \bar{H}^{*}(\Lambda(t))=-\frac{d}{d t} r^{*}(\Lambda(t)) \cdot \lambda(t) \\
=-\frac{d}{d t} r^{*}(\Lambda(t)) \cdot \lambda^{2}(t)-r^{*}(\Lambda(t)) \cdot \frac{d}{d t} \lambda(t) .
\end{gathered}
$$

Como $\bar{H}^{*}(t)$ é IFR, então $\frac{d}{d t} r^{*}(\Lambda(t)) \geq 0$, e $\frac{d}{d t} r(t)=\frac{d}{d t} r^{*}(\Lambda(t)) \geq 0$, e

$$
\frac{d^{2}}{d t^{2}} \log \bar{H}(t)=-\frac{d}{d t} r^{*}(\Lambda(t)) \cdot \lambda^{2}(t)-r^{*}(\Lambda(t)) \cdot \frac{d}{d t} \lambda(t) \leq 0 .
$$

Sob a Definição A.3.1, concluímos que T é IFR.

Além da classe de distribuição do D-espectro do network, a convexidade (concavidade) da função de risco do Processo de Poisson Não Homogêneo é indispensável. Não é difícil notar que esta propriedade significa que o instante da primeira falha dos links tem taxa de falha crescente.

Observação 3.1 Como $\Lambda(t)=-\log \bar{G}(t)$, onde $\bar{G}(t)=P\left(R_{1}>t\right)$ e $R_{1}$ é o instante da primeira falha dos links do network, temos que $\Lambda(t)$ convexa, consequentemente $\bar{G}$ é log-côncava e $R_{1}$ tem taxa de falha crescente.

Esta observação nos motiva a compreender claramente a importância da convexidade da função de risco do Processo de Poisson Não Homogêneo para as falhas dos links de um network.

O exemplo que segue mostra-nos a classe de distribuição do tempo de vida do network, ao desconsiderarmos a convexidade da função de risco (este exemplo tem autoria própria).

Exemplo 3.1.3 (vide Exemplo A.4.6) Considere um network com D-espectro $\mathbf{f}=(0,5 ; 0,2$; 0,3). Suponha que as falhas de seus links ocorrem segundo um Processo de Poisson Não Homogêneo $\{N(t): t>0\}$ com função de risco $\Lambda(t)=e^{t}$.

Sabemos, pelo Exemplo 3.1.1, que f é DFR.

A confiabilidade do network no instante $\mathrm{t}>0$ é

$$
\bar{H}(t)=P(T>t)=\sum_{k=0}^{2} \bar{F}_{k} \cdot P(N(t)=k)=e^{-e^{t}} \cdot\left(1+0,5 \cdot e^{t}+0,15 \cdot\left(e^{t}\right)^{2}\right) .
$$

Então

$$
\begin{gathered}
\log \bar{H}(t)=-e^{t}+\log \left(1+0,5 . e^{t}+0,15 \cdot\left(e^{t}\right)^{2}\right) \\
\frac{d}{d t} \log \bar{H}(t)=\frac{-0,5 \cdot e^{t}-0,2 . e^{2 t}-0,15 \cdot e^{3 t}}{1+0,5 \cdot e^{t}+0,15 \cdot\left(e^{2 t}\right)} \\
\frac{d^{2}}{d t^{2}} \log \bar{H}(t)=\frac{-0,5 . e^{t}-0,4 . e^{2 t}-0,475 . e^{3 t}-0,15 \cdot e^{4 t}-0,1125 . e^{5 t}}{\left(1+0,5 \cdot e^{t}+0,15 \cdot e^{2 t}\right)^{2}} \leq 0 .
\end{gathered}
$$


Logo, $\frac{d}{d t} \log \bar{H}(t)$ é decrescente em $\mathrm{t}>0$.

Observe que $\frac{d}{d t} \Lambda(t)=e^{t} \geq 0$ e, portanto, $\Lambda(t)=e^{t}$ é convexa.

Sob a Definição A.3.1, concluímos que T é IFR, sentido contrário à classe de distribuição do D-espectro $\mathbf{f}$.

Pode-se pensar de maneira intuitiva que o tempo de vida de um network como função crescente dos tempos de vidas de seus links, tenha a propriedade IFR se seus links são IFR. Contudo, tal pensamento é falso. Ou seja, um network com links IFR nem sempre é IFR, isto é, a coleção de networks não é fechada em relação à classe IFR (ver Barlow e Proschan (1975)). Contudo, podemos definir a classe de distribuições IFRA, que, no contexto desta dissertação, é preservada na formação do network.

Definição 3.1.2 (Barlow e Proschan, 1975) Considere um network com D-espectro f. Dizemos que f, ou sua distribuição, tem taxa de falha crescente (decrescente) em média - IFRA (DFRA) se

$$
-\frac{1}{k} \cdot \log \left(\bar{F}_{k}\right)
$$

é crescente (decrescente) em $\mathrm{k}=1,2 \ldots, \mathrm{n}$, onde $\bar{F}_{k}=\sum_{j=k+1}^{n} f_{j}$.

Equivalentemente, o D-espectro f é IFRA (DFRA) se $\bar{F}_{k}^{\frac{1}{k}}$ é decrescente (crescente) em k=1,2...,n.

De maneira prática, o D-espectro de um network tem taxa de falha crescente em média quando à medida que aumenta a quantidade de falhas de seus links a propensão do network a falhas aumenta em média. Pode-se mostrar que se uma distribuição é IFR, então é IFRA (ver Apêndice).

Exemplo 3.1.4 (ver Exemplo A.4.7) Considere o network e seu D-espectro vistos no Exemplo 3.1.1.

Vamos avaliar a classe IFRA (DFRA) para o D-espectro.

Considere $\bar{F}^{*}$ um vetor tal que, para este exemplo

$\bar{F}^{*}=\left(\bar{F}_{1}^{\frac{1}{1}}, \bar{F}_{2}^{\frac{1}{2}}\right)=\left(0,5^{\frac{1}{1}}, 0,3^{\frac{1}{2}}\right)=(0,5 ; 0,55)$.

Como $\bar{F}_{k}^{\frac{1}{k}}$ é crescente em $\mathrm{k}=1,2$, concluímos que f é DFRA.

Veremos um exemplo de preservação da classe IFRA.

Exemplo 3.1.5 (ver Exemplo A.4.8) Considere um network com D-espectro $\mathbf{f}=(0,5 ; 0,2$; 0,3), e que as falhas de seus links ocorrem de acordo com um Processo de Poisson Não Homogêneo com função de risco $\Lambda(t)=\sqrt{t}$.

Pelo Exemplo 3.1.4, sabemos que f é DFRA.

Verificamos se a função de risco é star-shaped (Definição A.3.5)

$$
\frac{d}{d t} \frac{\Lambda(t)}{t}=-\frac{1}{2} \cdot t^{-\frac{3}{2}} \leq 0
$$

Portanto, $\frac{\Lambda(t)}{t}$ é decrescente, e $\Lambda(t)$ é antistar-shaped .

A confiabilidade do network é 


$$
\bar{H}(t)=\sum_{k=0}^{2} \bar{F}_{k} \cdot P(N(t)=k)=e^{-\sqrt{t}}\left(1+\sqrt{t}+\frac{t}{2}\right) .
$$

Temos que

$$
\frac{\log \bar{H}(t)}{t}=t^{-\frac{1}{2}}+\frac{1}{t} \log \left(1+t^{\frac{1}{2}}+\frac{t}{2}\right)
$$

Portanto

$$
\frac{d}{d t} \frac{\log \bar{H}(t)}{t}=\frac{1}{2} \cdot t^{-\frac{3}{2}}+\frac{\frac{1}{2} \cdot t^{-\frac{1}{2}}+\frac{1}{2}}{1+t^{\frac{1}{2}}+\frac{t}{2}} \geq 0,
$$

e $\log \bar{H}(t)$ é star-shaped. Portanto T é DFRA (Definição A.3.2 e Observação A.3.2).

Enunciaremos o teorema de preservação da classe de distribuições IFRA (DFRA) do D-espectro de um network para seu tempo de vida. Observe que a definição da classe IFRA é equivalente a dizer que $-\log \bar{H}(t)$ é star-shaped (Definição A.3.5), onde $\bar{H}(t)$ é a confiabilidade do network no instante $t>0$. A motivação para enunciar e provar este teorema é verificar que o estudo da classe IFRA para o D-espectro e da propriedade star-shaped para a função de risco do Processo de Poisson Não Homogêneo para as falhas dos links nos basta para concluir que o network é IFRA.

Teorema 3.1.2 Seja T o tempo de vida de um network com função de distribuição contínua e Despectro f, cujas falhas de seus links ocorrem segundo um Processo de Poisson Não Homogêneo com função de risco $\Lambda(t)$. Se f é IFRA (DFRA) e $\Lambda(t)$ é star-shaped (respectivamente antistar-shaped) , entáo T é IFRA (DFRA).

Prova Provaremos a preservação da classe IFRA.

Primeiramente, considere um Processo de Poisson Homogêneo com taxa $\lambda>0$ (Teorema 3.1 parte (3.4) de Esary et al. (1973)). Neste caso, a confiabilidade do network no instante t $>0$ é.

$$
\bar{H}^{*}(t)=\sum_{k=0}^{n-1} \bar{F}_{k} \cdot \frac{e^{-\lambda \cdot t} \cdot(\lambda \cdot t)^{k}}{k !} .
$$

Por hipótese f é IFRA, isto é, $\bar{F}_{k}^{\frac{1}{k}}$ é decrescente em $\mathrm{k}=0, \ldots, \mathrm{n}-1$ (Definição 3.1.2.). Então, para $\xi \in(0,1)$ fixado, $\bar{F}_{k}^{\frac{1}{k}}-\xi$ tem no máximo uma troca de sinal, de positivo para negativo. Assim, $\bar{F}_{k}-\xi^{k}$ também possui no máximo uma troca de sinal de positivo para negativo em $\mathrm{k}$.

Como $\mathrm{P}(\mathrm{N}(\mathrm{t})=\mathrm{k})$ é $\mathrm{TP}_{2}$ em $\mathrm{t}>0$ e $\mathrm{k}=0,1,2 \ldots, \mathrm{n}-1$, usando a propriedade de diminuição da variação (ver seção A.3 do Apêndice),

$$
\begin{aligned}
& \sum_{k=0}^{\infty}\left(\bar{F}_{k}-\xi^{k}\right) e^{-\lambda . t} \frac{(\lambda . t)^{k}}{k !}=\sum_{k=0}^{\infty} \bar{F}_{k} \cdot e^{-\lambda . t} \frac{(\lambda . t)^{k}}{k !}-\sum_{k=0}^{\infty} \xi^{k} e^{-\lambda . t} \frac{(\lambda . t)^{k}}{k !} \\
= & \bar{H}^{*}(t)-\sum_{k=0}^{\infty} \xi^{k} e^{-\lambda . t} \frac{(\lambda . t)^{k}}{k !}=\bar{H}^{*}(t)-e^{-\lambda . t} \cdot e^{+\lambda . \xi . t}=\bar{H}^{*}(t)-e^{-(1-\xi) \cdot \lambda . t}
\end{aligned}
$$

tem no máximo uma troca de sinal de positivo para negativo.

Tome $\theta=(1-\xi) . \lambda<\lambda$. Então, $\bar{H}^{*}(t)-e^{-\theta . t}$ tem no máximo uma troca de sinal de positivo para negativo.

Se $\theta \geq \lambda$, então $e^{-\theta . t} \leq e^{-\lambda . t}, \mathrm{t}>0$. 
Desde que

$$
\bar{H}^{*}(t)=e^{-\lambda \cdot t}+\sum_{k=1}^{\infty} \bar{F}_{k} \cdot e^{-\lambda \cdot t} \frac{(\lambda \cdot t)^{k}}{k !} \geq e^{-\lambda \cdot t},
$$

Temos que $\bar{H}^{*}(t) \geq e^{-\theta \cdot t}, \mathrm{t}>0$

Concluímos que, para todo $\theta>0, \bar{H}(t)-e^{-\theta . t}$ tem no máximo uma troca de sinal de positivo para negativo. Assim, pelo (Teorema A.3.2), $\bar{H}^{*}(t)^{\frac{1}{t}}$ é decrescente. Portanto, sob a Definição A.3.2, $\bar{H}^{*}(t)$ é IFRA.

Considerando $\lambda=1$, representamos $\bar{H}(t)$ por $\bar{H}(t)=\bar{H}^{*}(\Lambda(t)), t>0$ (Teorema 3.1 (d) de A-Hameed e Proschan (1973)), onde

$$
\bar{H}^{*}(x)=\sum_{k=0}^{n-1} \bar{F}_{k} \cdot \frac{e^{-x} \cdot x^{k}}{k !} .
$$

Como f e $\bar{H}^{*}(t)$ é IFRA, $-\frac{1}{t} \log \bar{H}^{*}(t)$ é crescente (Definição A.3.2) e $-\log \bar{H}^{*}(t)$ é star-shaped. Como $\bar{H}^{*}(t)$ é decrescente em t, então $-\log \bar{H}^{*}(t)$ é crescente em $\mathrm{t}>0$.

Assim, para $\alpha \in(0,1)$,

$$
-\log \bar{H}(\alpha . t)=-\log \bar{H}^{*}(\Lambda(\alpha . t)) \leq-\log \bar{H}^{*}(\alpha . \Lambda(t)) \leq-\alpha \cdot \log \bar{H}^{*}(\Lambda(t))=-\alpha \cdot \log \bar{H}(t)
$$

A primeira desigualdade é válida, pois $\Lambda(t)$ é star-shaped e $-\log \bar{H}^{*}(t)$ é crescente em $\mathrm{t}>0$. A segunda também o é, pois $-\log \bar{H}^{*}(t)$ é star-shaped.

Como $-\log \bar{H}(t)$ é star-shaped, T é IFRA (Definições A.3.2 e A.3.5).

Além da classe IFRA (DFRA) para o D-espectro, é imprescindível considerar a propriedade star-shaped (ou antistar-shaped) para a função de risco do Processo de Poisson Não Homogêneo para as falhas dos links. Dizer que a função de risco é star-shaped significa que o instante da primeira falha dos links tem taxa de falha crescente em média.

Observação 3.1.2 Se a função de risco $\Lambda(t)=-\log \bar{G}(t)$ é star-shaped, então $\frac{-\log \bar{G}(t)}{t}$ é crescente em t, onde $\bar{G}(t)=P\left(R_{1}>t\right)$ portanto, sob a Definição A.3.2, $\bar{G}$ é IFRA e $R_{1}$ é IFRA.

Enunciamos um exemplo do que ocorre ao ignorarmos a propriedade para a função de risco (exemplo do próprio autor).

Exemplo 3.1.6 (ver Exemplo A.4.9) Considere um network com tempo de vida T e Despectro $\mathbf{f}=(0,5 ; 0,2 ; 0,3)$. Considere que as falhas de seus links ocorrem segundo um Processo de Poisson Não Homogêneo com função de risco $\Lambda(t)=t^{2}$. Queremos analisar a preservação da classe IFRA (DFRA) para este network.

Pelo Exemplo 3.1.4 sabemos que o D-espectro f é DFRA.

A confiabilidade do network no instante $\mathrm{t}>0$ é

$$
\bar{H}(t)=e^{-t^{2}}\left(1+0,5 \cdot t^{2}+0,15 \cdot t^{4}\right) .
$$

Temos:

$$
\frac{1}{t} \log \bar{H}(t)=\frac{1}{t} \cdot\left[-t^{2}+\log \left(1+0,5 \cdot t^{2}+0,15 \cdot t^{4}\right)\right]=-t+\frac{\log \left(1+0,5 \cdot t^{2}+0,15 \cdot t^{4}\right)}{t}
$$




$$
\frac{d}{d t} \frac{\log \bar{H}(t)}{t}=\frac{0,1 \cdot t^{4}-0,15 \cdot t^{6}-\left(1+0,5 \cdot t^{2}+0,15 \cdot t^{4}\right) \cdot \log \left(1+0,5 \cdot t^{2}+0,15 \cdot t^{4}\right)}{\left(1+0,5 \cdot t^{2}+0,15 \cdot t^{4}\right) \cdot t^{2}} .
$$

Como o denominador é positivo, é suficiente analisar o sinal do numerador.

Seja

$$
\phi(t)=0,1 \cdot t^{4}-0,15 \cdot t^{6}-\left(1+0,5 \cdot t^{2}+0,15 \cdot t^{4}\right) \cdot \log \left(1+0,5 \cdot t^{2}+0,15 \cdot t^{4}\right) .
$$

Temos: $\phi(0)=0 \mathrm{e}$

$$
\frac{d \phi(t)}{d t}=-t-0,2 t^{3}-0,9 t^{5}-\left(t+0,6 t^{3}\right) \cdot \log \left(1+0,5 \cdot t^{2}+0,15 \cdot t^{4}\right) \leq 0 .
$$

Então $\phi(t) \leq 0$ e portanto

$$
\frac{d}{d t} \frac{\log \bar{H}(t)}{t} \leq 0
$$

Concluímos que $\frac{1}{t} \log \bar{H}(t)$ é decrescente em t e T é IFRA (Definição A.3.2), em sentido contrário ao D-espectro $\mathbf{f}$.

Observe que

$$
\frac{\Lambda(t)}{t}=\frac{t^{2}}{t}=t
$$

é crescente em $\mathrm{t}>0$. Portanto, a função de risco $\Lambda(t)=t^{2}$ é star-shaped. Tal condição não implica a preservação da classe do D-espectro para o tempo de vida.

Outras classes de distribuições usadas em políticas de manutenção são as classes NBU (NWU) e NBUE (NWUE).

Definição 3.1.3 (Barlow e Proschan, 1975) Considere um network com n componentes e f seu D-espectro. Dizemos que o D-espectro f, é novo melhor (pior) do que usado - NBU (NWU) se, para $k$ e $j=1 \ldots n$.

$$
\bar{F}_{k+l} \leq(\geq) \bar{F}_{k} \bar{F}_{l}
$$

Dizer que uma distribuição é NBU é equivalente a afirmar que a probabilidade de sobrevivência condicionada à certa idade é menor do que a probabilidade de sobrevivência de uma unidade nova. É claro que a classe de distribuição NBU inclui a classe de distribuições IFR e que em tais casos é recomendável o uso de uma política de manutenção e de reparos. Pode-se mostrar que se uma distribuição é IFRA, então é NBU (ver Teorema A.3.3 parte (ii)).

Exemplo 3.1.7 (ver Exemplo A.4.10) Considere um network com D-espectro $\mathbf{f}=(0,5 ; 0,2$; $0,3)$.

Verificamos se o D-espectro é NBU. Como $\bar{F}_{2}-\bar{F}_{1} \cdot \bar{F}_{1}=0,3-0,5.0,5=0,05>0$, o D-espectro é NWU.

Veremos um exemplo da preservação da classe NBU (NWU).

Exemplo 3.1.8 (ver Exemplo A.4.11) Seja T o tempo de vida de um network com Despectro $\mathbf{f}=(0,1 ; 0,3 ; 0,6)$, cujas falhas de seus links ocorrem de acordo com um Processo de Poisson Homogêneo com taxa $\lambda(t)=1$

Verificamos que o D-espectro é NBU. Como $\bar{F}_{2}-\bar{F}_{1} \cdot \bar{F}_{1}=0,6-0,9.0,9=-0,21 \leq 0, \mathbf{f}$ é NBU (Definição 3.1.3). 
Observe que

$$
\Lambda(t+x)=\lambda \cdot t+\lambda \cdot x=\Lambda(t)+\Lambda(x),
$$

ou seja, $\Lambda(t)=\lambda$.t é superaditiva e subaditiva (Definição A.3.6).

A confiabilidade do network é

$$
\bar{H}(t)=\sum_{k=0}^{2} \bar{F}_{k} \cdot P(N(t)=k)=e^{-t}\left(1+0,9 \cdot t+0,3 \cdot t^{2}\right) .
$$

Concluímos que

$$
\bar{H}(t+x)-\bar{H}(t) \bar{H}(x)=-0,21 t x-0,27 t^{2} x-0,27 t x^{2}-0,09 t^{2} x^{2} \leq 0,
$$

e, portanto, sob a Definição A.3.3, T é NBU.

Enunciamos o teorema de preservação da classe de distribuições NBU do D-espectro para o tempo de vida de um network. Estamos interessados em provar que a análise da classe NBU para o D-espectro e da superaditividade da função de risco são suficientes para deduzir que o tempo de vida do network é NBU.

Teorema 3.1.3 Seja T o tempo de vida de um network com função de distribuição contínua e D-espectro f, cujas falhas de seus links ocorrem segundo um Processo de Poisson Não Homogêneo com função de risco $\Lambda(t)$. Se f é NBU (NWU) e $\Lambda(t)$ é superaditiva (subaditiva), então T é NBU (NWU).

Prova Verificaremos a preservação da classe NBU.

Inicialmente, considere um Processo de Poisson Homogêneo com taxa $\lambda>0$ (Teorema 3.2 parte (3.5) de Esary et al. (1973)). Neste caso, a confiabilidade do network no instante t $>0$ é

$$
\bar{H}^{*}(t)=\sum_{k=0}^{n-1} \bar{F}_{k} \cdot \frac{e^{-\lambda \cdot t} \cdot(\lambda . t)^{k}}{k !} .
$$

Basta verificar que, para $\mathrm{x}>0$ e $\mathrm{t}>0$,

$$
\bar{H}^{*}(x) \bar{H}^{*}(t)-\bar{H}^{*}(x+t) \leq 0,
$$

onde $\bar{H}^{*}(t)$ é a confiabilidade do network, cujas falhas de seus links ocorrem segundo um Processo de Poisson Homogêneo com taxa $\lambda>0$.

Assim

$$
\begin{gathered}
\bar{H}^{*}(x) \bar{H}(t)=\sum_{k=0}^{n-1} \sum_{j=0}^{n-1} \bar{F}_{k} \cdot P(N(x)=k) \cdot \bar{F}_{j} \cdot P(N(t)=j) \\
=\sum_{k=0}^{n-1} \sum_{j=0}^{n-1} \bar{F}_{k} \cdot \bar{F}_{j} \cdot \frac{e^{-\lambda \cdot x} \cdot(\lambda \cdot x)^{k}}{k !} \cdot \frac{e^{-\lambda \cdot t} \cdot(\lambda \cdot t)^{j}}{j !} \leq \sum_{k=0}^{n-1} \sum_{j=0}^{n-1} \bar{F}_{k+j} \cdot e^{-\lambda \cdot(x+t)} \cdot \frac{(\lambda \cdot x)^{k} \cdot(\lambda \cdot t)^{j}}{k ! j !} .
\end{gathered}
$$

A desigualdade ocorre pois f é NBU, ou seja, $\bar{F}_{k} \cdot \bar{F}_{j} \leq \bar{F}_{k+j}$, para k,j=1..,n (Definição 3.1.3). 
Aplicando $\mathrm{l}=\mathrm{k}+\mathrm{j}$, temos

$$
\bar{H}^{*}(x) \bar{H}^{*}(t) \geq \sum_{k=0}^{n-1} \sum_{l=k}^{n-1+k} \bar{F}_{l} \cdot e^{-\lambda \cdot(x+t)} \cdot \frac{(\lambda \cdot x)^{k} \cdot(\lambda \cdot t)^{l-k}}{k !(l-k) !}=\sum_{l=0}^{2(n-1)} \sum_{k=0}^{l} \bar{F}_{l} \cdot e^{-\lambda \cdot(x+t)} \cdot \frac{(\lambda \cdot x)^{k} \cdot(\lambda \cdot t)^{l-k}}{k !(l-k) !} .
$$

Como $\bar{F}_{k}=0$, para $\mathrm{k}=\mathrm{n}, \mathrm{n}+1, \ldots 2(\mathrm{n}-1)$, temos:

$$
\begin{aligned}
\bar{H}^{*}(x) \bar{H}^{*}(t) & \geq \sum_{l=0}^{n-1} \bar{F}_{l} \cdot e^{-\lambda \cdot(x+t)} \cdot \frac{1}{l !} \cdot(\lambda(x+t))^{l} \sum_{k=0}^{l} \frac{(\lambda \cdot x)^{k} \cdot(\lambda \cdot t)^{l-k}}{(\lambda \cdot(x+t))^{l} \cdot k !(l-k) !} \cdot l ! \\
& =\sum_{l=0}^{n-1} \bar{F}_{l} \cdot e^{-\lambda \cdot(x+t)} \cdot \frac{1}{l !} \cdot(\lambda(x+t))^{l}=\bar{H}^{*}(x+t) .
\end{aligned}
$$

A penúltima igualdade resulta do Binômio de Newton. Sob a Definição A.3.3, concluímos que $\bar{H}^{*}(t)$ é NBU.

Tome $\lambda=1$ e considere as representações $\bar{H}$ e $\bar{H}^{*}$ do Teorema 3.1.1 (mais detalhes no Teorema 3.1 parte (f) de A-Hameed e Proschan (1973)).

Provamos que se f é NBU, então $\bar{H}^{*}(t)$ é NBU, ou seja,

$$
\bar{H}^{*}(x+y) \leq \bar{H}^{*}(x) \cdot \bar{H}^{*}(y) \Longleftrightarrow-\log \bar{H}^{*}(x+y) \geq-\left(\log \bar{H}^{*}(x)+\log \bar{H}^{*}(y)\right),
$$

ou seja $-\log \bar{H}^{*}(x)$ é superaditiva em x $>0$ (Definição A.3.6).

Assim

$$
\begin{aligned}
& -\log \bar{H}(x+y)=-\log \bar{H}^{*}(\Lambda(x+y)) \geq-\log \bar{H}^{*}(\Lambda(x)+\Lambda(y)) \\
& \geq-\left(\log \bar{H}^{*}(\Lambda(x))+\log \bar{H}^{*}(\Lambda(y))\right)=-(\log \bar{H}(x)+\log \bar{H}(y)) .
\end{aligned}
$$

A primeira desigualdade vale, pois $\Lambda(t)$ é superaditiva e $\bar{H}^{*}(t)$ é decrescente. A segunda é válida, pois $-\log \bar{H}^{*}(t)$ é superaditiva. Então $-\log \bar{H}(t)$ é superaditiva e T é NBU (Definição A.3.3).

A superaditividade ou subaditividade da função de risco do Processo de Poisson Não Homogêneo das falhas dos links de um network deve ser considerada juntamente à classe NBU ou NWU para o D-espectro do network.

Observação 3.1.3 Se a função de risco $\Lambda(t)=-\log \bar{G}(t)$ é superaditiva, onde $\bar{G}(t)=P\left(R_{1}>t\right)$, e $R_{1}$ é o instante da primeira falha dos links do network, então, para t e x reais positivos

$$
\begin{aligned}
& \Lambda(t+x) \geq \Lambda(t)+\Lambda(x) \Rightarrow-\log \bar{G}(t+x) \geq-\log \bar{G}(t)-\log \bar{G}(x) \\
& \Rightarrow-\log \bar{G}(t+x) \geq-\log (\bar{G}(t) \cdot \bar{G}(x)) \Rightarrow \bar{G}(t+x) \leq \bar{G}(t) \cdot \bar{G}(x) .
\end{aligned}
$$

Portanto, $\bar{G}$ é NBU e $R_{1}$ é NBU.

Concluímos que se a função de risco é superaditiva, o instante da primeira falha dos links de um network tem a propriedade do melhor novo do que usado. Esta observação nos fornece um entendimento mais lúcido de que não é suficiente que o D-espectro seja NBU para que o tempo de vida do network também o seja. 
Apresentamos um exemplo de network em que a superaditividade da função de risco não é considerada. O exemplo é desenvolvido pelo próprio autor.

Exemplo 3.1.9 (vide Exemplo A.4.12) Considere um network com D-espectro $\mathbf{f}=(0,5 ; 0,2$; $0,3)$ e T seu tempo de vida e que as falhas de seus links ocorrem segundo um Processo de Poisson Não Homogêneo com função de risco $\Lambda(t)=t^{2}$. Queremos estudar a preservação da classe NBU (NWU) para o network.

Segundo o Exemplo 3.1.7, o D-espectro f é NWU.

A confiabilidade do network no instante $\mathrm{t}>0$ é

$$
\bar{H}(t)=e^{-t^{2}}\left(1+0,5 \cdot t^{2}+0,15 \cdot t^{4}\right) .
$$

Tome $t_{3}=1, t_{1}=0,3$ e $t_{2}=0,7$.

Assim

$$
P(T>1)-P(T>0,3) . P(T>0,7)=-0,81<0 .
$$

Então, existem $t_{3}=1, t_{1}=0,3$ e $t_{2}=0,7$, com $t_{3}=t_{1}+t_{2}$ tais que

$$
P\left(T>t_{3}\right)-P\left(T>t_{1}\right) \cdot P\left(T>t_{2}\right) \leq 0 \text {. }
$$

Observe que a função de risco $\Lambda(t)=t^{2}$ é superaditiva, ou seja, para todo $\mathrm{x}, \mathrm{y}>0 \Lambda(x+y)=$ $(x+y)^{2}=x^{2}+y^{2}+2 \cdot x \cdot y \geq x^{2}+y^{2}=\Lambda(x)+\Lambda(y)$.

Portanto T não é NWU (Definição A.3.3 e Observação A.3.3), diferentemente à classe do Despectro $\mathbf{f}$.

Em nosso contexto, a classe de distribuições NBU é fechada na formação de networks, no sentido de que o tempo de vida de um network cujos tempos de vidas de seus links sejam NBU também terá essa propriedade. No entanto, em um network cuja confiabilidade é uma mistura das distribuições dos instantes de falhas dos links a preservação da classe NBU a partir de seu D-espectro depende da superaditividade de função de risco.

Outra classe de distribuições indispensável em políticas de manutenção com base na idade é a classe NBUE.

Definição 3.1.4 (Barlow e Proschan, 1975) Considere um network com n componentes e f seu D-espectro. Dizemos que f é novo melhor (pior) do que usado, em média - NBUE (NWUE), para $\mathrm{k}=1, \ldots \mathrm{n}$ se

(i) $\mu=\sum_{k=1}^{n} \bar{F}_{k}<\infty$;

(ii) $\bar{F}_{k} \cdot \mu \geq(\leq) \sum_{j=k}^{n} \bar{F}_{j-1}$.

Exemplo 3.10 (ver Exemplo A.4.13) Considere um network com D-espectro $\mathbf{f}=(0,5 ; 0,2$; $0,3)$.

Analisamos a preservação da classe NBUE (NWUE). Observe que

(i) $\mu=\sum_{j=1}^{3} \bar{F}_{j}=1,8<\infty$;

(ii) Avaliamos o sinal de $\sum_{j=k}^{3} \bar{F}_{j-1}-\mu \cdot \bar{F}_{k}$ para $\mathrm{k}=1,2,3$.

Para $\mathrm{k}=1$ 
$\sum_{j=1}^{3} \bar{F}_{j-1}-\mu \cdot \bar{F}_{1}=1,8-1,8.0,5=0,9>0$

Para $\mathrm{k}=2$

$\sum_{j=2}^{3} \bar{F}_{j-1}-\mu \cdot \bar{F}_{2}=0,8-1,8.0,3=0,26>0 ;$

Para $\mathrm{k}=3$

$\sum_{j=3}^{3} \bar{F}_{j-1}-\mu \cdot \bar{F}_{3}=0,3-1,8.0=0,3>0$.

Assim, $\sum_{j=k}^{3} \bar{F}_{j-1}-\mu \cdot \bar{F}_{k}>0$, para $\mathrm{k}=1,2,3$.

Portanto o D-espectro f é NWUE.

Dizer que uma distribuição é NBUE equivale a afirmar que o tempo de vida médio residual condicionado à determinada idade é menor do que o tempo de vida médio de uma unidade nova. Pode-se mostrar que se uma distribuição é NBU, então é NBUE (Teorema A.3.3 (iii)). A classe NBUE não é fechada na formação de networks. Contudo existem exemplos em que tal propriedade é satisfeita.

Veremos um exemplo da preservação da classe NBUE.

Exemplo 3.1.11 (vide Exemplo A.4.14) Seja $T$ o tempo de vida de um network com Despectro $\mathbf{f}=(0,1 ; 0,3 ; 0,6)$, cujas falhas de seus links ocorrem segundo um Processo de Poisson Homogêneo com taxa $\lambda(t)=1$.

Do Exemplo 3.1.8, sabemos que f é NBU, o que implica, pelo Teorema A.3.3 (iii), que f é NBUE.

Observe que

$$
\frac{\Lambda(t+x)}{t+x}-\frac{\Lambda(t)}{t}=\lambda-\lambda=0,
$$

e a função de risco $\Lambda(t)=\lambda . t$ é star-shaped e antistar-shaped (Definição A.3.5).

A confiabilidade do network é

$$
\bar{H}(t)=\sum_{k=0}^{2} \bar{F}_{k} \cdot P(N(t)=k)=e^{-t}\left(1+0,9 t+0,3 t^{2}\right) .
$$

O tempo médio do network é

$$
\mu=E(T)=\int_{0}^{\infty} \bar{H}(t) d t=1+0,9+0,6=2,5 .
$$

Vamos verificar que $\bar{H}(t) \cdot \mu \geq \int_{t}^{\infty} \bar{H}(s) d s$.

Observe que

$$
\begin{gathered}
\int_{t}^{\infty} \bar{H}(s) d s=\int_{t}^{\infty} e^{-s}\left(1+0,9 s+0,3 s^{2}\right) d s \\
=2,5 \cdot e^{-t}\left(1+0,6 \cdot t+0,12 \cdot t^{2}\right) \leq \mu \cdot e^{-t}\left(1+0,9 t+0,3 t^{2}\right)=\mu \cdot \bar{H}(t) .
\end{gathered}
$$

Sob a Definição A.3.4, concluímos que T é NBUE.

Analisaremos a preservação da classe de distribuição NBUE (NWUE) do D-espectro de um network para seu tempo de vida. Nossa motivação para enunciar e provar este teorema é constatar que basta estudar a classe NBUE para o D-espectro e a propriedade star-shaped para a função de risco do PPNH para as falhas dos seus links para deduzir a classe de distribuições para o network. 
Teorema 3.1.4 Seja T o tempo de vida de um network com função de distribuição contínua e D-espectro f, cujas falhas de seus links ocorrem segundo um Processo de Poisson Não Homogêneo com função de risco $\Lambda(t)$. Se f é NBUE (NWUE) e $\Lambda(t)$ é (anti)star-shaped, então T é NBUE (NWUE).

Prova Provamos a preservação da classe NBUE.

Primeiramente, considere um Processo de Poisson Homogêneo com taxa $\lambda>0$ (Teorema 3.1 parte (3.6) de Esary et al. (1973)). Neste caso, a confiabilidade do network no instante t $>0$ é

$$
\bar{H}^{*}(t)=\sum_{k=0}^{n-1} \bar{F}_{k} \cdot \frac{e^{-\lambda . t} \cdot(\lambda . t)^{k}}{k !} .
$$

Considere inicialmente o caso $\Lambda(t)=\lambda . t, \lambda>0$.

(i) Seja

$$
\begin{gathered}
\mu^{*}=\int_{0}^{\infty} \bar{H}^{*}(t) d t=\int_{0}^{\infty} \sum_{k=0}^{n-1} \bar{F}_{k} \cdot P(N(t)=k) d t \\
=\sum_{k=0}^{n-1} \bar{F}_{k} \cdot \int_{0}^{\infty} \frac{e^{-\lambda \cdot t} \cdot(\lambda \cdot t)^{k}}{k !} d t=\sum_{k=0}^{n-1} \frac{\bar{F}_{k}}{\lambda} \cdot \int_{0}^{\infty} \frac{e^{-\lambda \cdot t} \cdot(\lambda \cdot t)^{k} \cdot \lambda}{k !} d t=\sum_{k=0}^{n-1} \frac{\bar{F}_{k}}{\lambda}<\infty,
\end{gathered}
$$

onde $\bar{H}^{*}(t)$ é a confiabilidade de um network, cujas falhas de seus links ocorrem segundo um Processo de Poisson Homogêneo com taxa $\lambda>0$.

A penúltima igualdade resulta da integração da função densidade de probabilidade da distribuição $\operatorname{Gama}(\mathrm{k}+1, \lambda)$ em $(0, \infty)$ e o valor da somatória é finito pois f é NBUE, então $\sum_{k=0}^{n-1} \bar{F}_{k}<\infty$.

Portanto $\bar{H}^{*}(t)$ satisfaz a condição (i) da classe NBUE.

(ii) Basta verificar que $\bar{H}^{*}(t) \cdot \mu^{*}-\int_{t}^{\infty} \bar{H}^{*}(x) d x \leq 0$.

$$
\begin{gathered}
\bar{H}^{*}(t) \cdot \mu^{*}-\int_{t}^{\infty} \bar{H}^{*}(x) d x=\sum_{k=0}^{n-1} \bar{F}_{k} \cdot P(N(t)=k) \cdot \frac{\sum_{j=0}^{n-1} \bar{F}_{j}}{\lambda}-\int_{t}^{\infty} \sum_{k=0}^{n-1} \bar{F}_{k} \cdot P(N(x)=k) d x \\
=\sum_{k=0}^{n-1} \bar{F}_{k} \cdot \frac{e^{-\lambda \cdot t} \cdot(\lambda \cdot t)^{k}}{k !} \cdot \frac{\sum_{j=0}^{n-1} \bar{F}_{j}}{\lambda}-\sum_{k=0}^{n-1} \bar{F}_{k} \cdot \int_{t}^{\infty} \frac{e^{-\lambda \cdot x} \cdot(\lambda \cdot x)^{k}}{k !} d x \\
=\frac{1}{\lambda} \sum_{k=0}^{n-1} \frac{e^{-\lambda \cdot t} \cdot(\lambda \cdot t)^{k}}{k !}\left[\bar{F}_{k} \cdot \sum_{j=0}^{n-1} \bar{F}_{j}-\sum_{j=k}^{n-1} \bar{F}_{j}\right] \leq 0 .
\end{gathered}
$$

A última igualdade resulta de

$$
\begin{gathered}
\int_{t}^{\infty} \frac{e^{-\lambda \cdot x} \cdot(\lambda \cdot x)^{k}}{k !} d x=\frac{\Gamma(k+1, \lambda \cdot t)}{\Gamma(k+1)} \\
=P\left(R_{k+1}>t\right)=P(N(t)<k+1)=P(N(t) \leq k)=\sum_{j=0}^{k} \frac{e^{-\lambda \cdot t} \cdot(\lambda \cdot t)^{j}}{j !} .
\end{gathered}
$$

Como f é NBUE, satisfaz a condição (ii) da classe NBUE. Portanto $\bar{H}^{*}(t)$ é NBUE, sob a Definição A.3.4.

Baseamo-nos agora no Teorema 3.1 parte (6) de A-Hameed e Proschan (1973). 
(i) Considere $\mu=E(T)$, e $\bar{H}(t)=\bar{H}^{*}(\Lambda(t))$ sob o Teorema 3.1.1.

Provamos que se f é NBUE, então $\bar{H}^{*}(t)$ é NBUE, e satisfaz a condição (i), ou seja, $\int_{0}^{\infty} \bar{H}^{*}(t) d t<$ $\infty$.

Como o Processo de Poisson Não Homogêneo para as falhas dos links do network é um processo de contagem não-explosivo, $\mu=\int_{0}^{\infty} \bar{H}(t) d t=\int_{0}^{\infty} \bar{H}^{*}(\Lambda(t)) d t<\infty$, e T satisfaz a condição (i).

(ii) Temos que

$$
\begin{gathered}
\bar{H}^{*}(\Lambda(t)) \cdot \int_{0}^{\infty} \bar{H}^{*}(\Lambda(x)) d x-\int_{t}^{\infty} \bar{H}^{*}(\Lambda(x)) d x \\
=\bar{H}^{*}(\Lambda(t)) \cdot \int_{0}^{t} \bar{H}^{*}(\Lambda(x)) d x-H^{*}(\Lambda(t)) \cdot \int_{t}^{\infty} \bar{H}^{*}(\Lambda(x)) d x \\
\geq \bar{H}^{*}(\Lambda(t)) \cdot \int_{0}^{t} \bar{H}^{*}\left(\Lambda\left(x \cdot \frac{\Lambda(t)}{t}\right)\right) d x-H^{*}(\Lambda(t)) \cdot \int_{t}^{\infty} \bar{H}^{*}\left(\Lambda\left(x \cdot \frac{\Lambda(t)}{t}\right)\right) d x .
\end{gathered}
$$

A igualdade vale, pois $\bar{H}^{*}(t)+H^{*}(t)=1$ e a desigualdade é válida, pois $\Lambda(t)$ é star-shaped (Definição A.3.5) e $\bar{H}^{*}(t)$ é decrescente. Fazendo a mudança de variável $y=\frac{x \cdot \Lambda(t)}{t}$, temos:

$$
\begin{aligned}
= & \frac{t}{\Lambda(t)}\left[\bar{H}^{*}(\Lambda(t)) \cdot \int_{0}^{\Lambda(t)} \bar{H}^{*}(y) d y-H^{*}(\Lambda(t)) \cdot \int_{\Lambda(t)}^{\infty} \bar{H}^{*}(y) d y\right] \\
= & \frac{t}{\Lambda(t)}\left[\bar{H}^{*}(\Lambda(t)) \cdot \int_{0}^{\Lambda(t)} \bar{H}^{*}(y) d y-H^{*}(\Lambda(t)) \cdot \int_{\Lambda(t)}^{\infty} \bar{H}^{*}(y) d y\right. \\
& \left.-\bar{H}^{*}(\Lambda(t)) \cdot \int_{\Lambda(t)}^{\infty} \bar{H}^{*}(y) d y+\bar{H}^{*}(\Lambda(t)) \cdot \int_{\Lambda(t)}^{\infty} \bar{H}^{*}(y) d y\right] \\
= & \frac{t}{\Lambda(t)}\left[\bar{H}^{*}(\Lambda(t)) \cdot \int_{0}^{\Lambda(t)} \bar{H}^{*}(y) d y+\bar{H}^{*}(\Lambda(t)) \cdot \int_{\Lambda(t)}^{\infty} \bar{H}^{*}(y) d y\right. \\
& \left.-H^{*}(\Lambda(t)) \cdot \int_{\Lambda(t)}^{\infty} \bar{H}^{*}(y) d y--\bar{H}^{*}(\Lambda(t)) \cdot \int_{\Lambda(t)}^{\infty} \bar{H}^{*}(y) d y\right] \\
=\frac{t}{\Lambda(t)}[ & \left.\bar{H}^{*}(\Lambda(t)) \cdot \int_{0}^{\infty} \bar{H}^{*}(y) d y-\left(H^{*}(\Lambda(t))+\bar{H}^{*}(\Lambda(t))\right) \cdot \int_{\Lambda(t)}^{\infty} \bar{H}^{*}(y) d y\right] \\
& =\frac{t}{\Lambda(t)}\left[\bar{H}^{*}(\Lambda(t)) \cdot \int_{0}^{\infty} \bar{H}^{*}(y) d y-\int_{\Lambda(t)}^{\infty} \bar{H}^{*}(y) d y\right]=0 .
\end{aligned}
$$

Estas igualdades são válidas pois somamos integrais sobre os intervalos disjuntos $(0, \mathrm{t})$ e $(\mathrm{t}, \infty)$ e $\bar{H}^{*}(\Lambda(t))+H^{*}(\Lambda(t))=1$.

Concluímos que $\bar{H}(t) \cdot \int_{0}^{\infty} \bar{H}(x) d x \geq \int_{t}^{\infty} \bar{H}(x) d x$ e, sob a Definição A.3.4, T é NBUE.

Observação 3.1.4 Se a função de risco $\Lambda(t)=-\log \bar{G}(t)$ é star-shaped, vimos na Observação 3.1.2 que $\bar{G}$ é IFRA. Sob o Teorema A.3.3, $\bar{G}$ é NBU, e consequentemente NBUE. Portanto, $R_{1}$, o instante da primeira falha dos links do network pertence à classe do novo melhor do que usado em média. 
Com o Teorema 3.1.4, podemos dizer que o uso indiscriminado da função de risco $\Lambda(t)$ não garante a preservação da classe NBUE (NWUE) entre o D-espectro e o tempo de vida do network cujos links falham segundo um Processo de Poisson Não Homogêneo. Observamos o exemplo, do próprio autor, a seguir.

Exemplo 3.1.12 (vide Exemplo A.4.15) Considere um network com D-espectro $\mathbf{f}=(0,5$; 0,2; 0,3$)$ e tempo de vida $\mathrm{T}$ e que as falhas de seus links ocorrem segundo um Processo de Poisson Não Homogêneo com função de risco $\Lambda(t)=e^{t}, \mathrm{t}>0$.

Queremos avaliar a preservação da classe de distribuição NBUE (NWUE).

Observe que

$$
\frac{d \Lambda(t)}{d t}=\frac{e^{t}}{t^{2}}(t-1)
$$

Concluímos que $\frac{d \Lambda(t)}{d t} \geq(\leq) 0 \Longleftrightarrow t \geq(\leq) 1$.

Portanto, $\Lambda(t)$ é antistar-shaped para $t \leq 1$ e star-shaped para $t \geq 1$ (Definição A.3.5).

Segundo o Exemplo 3.1.10, o D-espectro f é NWUE.

A confiabilidade do network no instante $\mathrm{t}>0$ é

$$
\bar{H}(t)=e^{-e^{t}} \cdot\left(1+0,5 \cdot e^{t}+0,15 \cdot e^{2 t}\right) .
$$

Verificamos a parte (i) da classe NBUE (NWUE).

O tempo médio de vida do network é

$$
\mu=E(T)=\int_{0}^{\infty} \bar{H}(t) d t=\int_{0}^{\infty} e^{-e^{t}} \cdot\left(1+0,5 \cdot e^{t}+0,15 \cdot e^{2 t}\right) d t
$$

Aplicando a mudança de variável $v=e^{t}$, temos

$$
\mu=\int_{1}^{\infty} e^{-v} \cdot\left(1+0,5 \cdot v+0,15 \cdot v^{2}\right) \frac{d v}{v}=E i(-1)+0,8 \cdot e^{-1} \approx 0,22+0,8 \cdot e^{-1} \approx 0,51<\infty,
$$

onde Ei(.) é a Função Exponencial Integral (Definição A.3.9).

(ii)

Considere $t_{0}=2$. Então

$$
\begin{gathered}
\bar{H}(2)=e^{-e^{2}}\left(1+0,5 \cdot e^{2}+0,15 \cdot e^{4}\right) \approx 0,08 \\
\int_{2}^{\infty} \bar{H}(t) d t=\int_{2}^{\infty} e^{-e^{t}} \cdot\left(1+0,5 \cdot e^{t}+0,15 \cdot e^{2 t}\right) d t=E i\left(-e^{2}\right)+0,001 \approx 0,00107 .
\end{gathered}
$$

Portanto

$$
\int_{2}^{\infty} \bar{H}(t) d t-\mu \cdot \bar{H}(2)=0,00107-0,51.0,008 \approx-0,003<0 .
$$

Então existe $t_{0}=2$ tal que $\int_{2}^{\infty} \bar{H}(t) d t-\mu \cdot \bar{H}(2)<0$ e, portanto, T não é NWUE (Definição A.3.4), diferentemente do D-espectro $\mathbf{f}$. 
Concluímos que em um network cujos links estão sujeitos a falhas de acordo com um Processo de Poisson Não Homogêneo, a preservação das classes de distribuições do D-espectro do network depende de restrições adequadas sobre a função de risco do processo.

\subsection{Resultados de equivalências das classes de distribuições do D- espectro para o D-espectro condicional de um network}

Veremos um exemplo de equivalências das classes de distribuições do D-espectro para o Despectro condicional de um network.

Exemplo 3.2.1 (vide Exemplo A.4.16, de autoria própria Seja $\mathbf{f}=(0,1 ; 0,3 ; 0,6)$ o Despectro de um network com tempo de vida $\mathrm{T}$, cujas falhas de seus links ocorrem segundo um Processo de Poisson Não Homogêneo com função de risco $\Lambda(t)=t^{2}$. Seja $\mathbf{p}(\mathbf{t})$ seu D-espectro condicional no instante t. Queremos analisar a equivalência das classes de distribuições do Despectro para o D-espectro condicional para t $>0$, fixado.

Seja $\bar{F}_{k}$ a função de sobrevivência do D-espectro $\mathbf{f}$.

Temos que $\bar{F}_{0}=1 ; \bar{F}_{1}=0,9 ;$ e $\bar{F}_{2}=0,6$;

Verificaremos se $\mathbf{f}$ é IFR.

Como $\bar{F}_{2}^{2}-\bar{F}_{1} \cdot \bar{F}_{3}=0,9^{2}-1.0,3=0,21 \geq 0$, concluímos que $\bar{F}_{k+l} \cdot \bar{F}_{k+1}-\bar{F}_{k+1+l} \cdot \bar{F}_{k} \geq 0$, para todo $\mathrm{k}, \mathrm{l}=1,2, \ldots 5$, e, portanto, f é IFR (Definição 3.1.1).

Segundo o Teorema A.3.3, f é IFRA, NBU e NBUE.

O k-ésimo componente do D-espectro condicional em t é

$$
p_{k}(t)=\frac{f_{k} \cdot P\left(R_{k}>t\right)}{P(T>t)} .
$$

A confiabilidade do network é

$$
P(T>t)=e^{-t^{2}}\left(1+0,9 t^{2}+\frac{0,6}{2} t^{4}\right)=e^{-t^{2}}\left(1+0,9 t^{2}+0,3 t^{4}\right) .
$$

Temos

$$
\begin{gathered}
P\left(R_{1}>t\right)=e^{-t^{2}} ; \\
P\left(R_{2}>t\right)=e^{-t^{2}}\left(1+t^{2}\right) ; \\
P\left(R_{3}>t\right)=e^{-t^{2}}\left(1+t^{2}+\frac{t^{4}}{2}\right) ;
\end{gathered}
$$

Portanto

$$
\begin{aligned}
p_{1}(t) & =\frac{0,1}{1+0,9 t^{2}+0,3 t^{4}} \\
p_{2}(t) & =\frac{0,3+0,3 t^{2}}{1+0,9 t^{2}+0,3 t^{4}} \\
p_{3}(t) & =\frac{0,6+0,6 t^{2}+0,3 t^{4}}{1+0,9 t^{2}+0,3 t^{4}}
\end{aligned}
$$


Além disso

$$
\begin{gathered}
\sum_{k=1}^{3} p_{k}(t)=1 \\
\sum_{k=2}^{3} p_{k}(t)=\frac{0,9+0,9 t^{2}+0,3 t^{4}}{1+0,9 t^{2}+0,3 t^{4}} \\
\sum_{k=3}^{3} p_{k}(t)=\frac{0,6+0,6 t^{2}+0,3 t^{4}}{1+0,9 t^{2}+0,3 t^{4}} .
\end{gathered}
$$

Verificamos que $\mathbf{p}(\mathbf{t})$ é IFR, ou seja, $\frac{\sum_{k=j+1}^{3} p_{k}(t)}{\sum_{k=j}^{3} p_{k}(t)}$ é decrescente em j, isto é

$$
\left(\sum_{k=j}^{3} p_{k}(t)\right)^{2}-\sum_{k=j-1}^{3} p_{k}(t) \cdot \sum_{k=j+1}^{3} p_{k}(t) \geq 0
$$

Então

$$
\left(\sum_{k=2}^{3} p_{k}(t)\right)^{2}-\sum_{k=1}^{3} p_{k}(t) \cdot \sum_{k=3}^{3} p_{k}(t)=\frac{0,21+0,48 t^{2}+0,33 t^{4}+0,09 t^{6}}{1+0,9 t^{2}+0,3 t^{4}} \geq 0 .
$$

Concluímos que p(t) é IFR (Definição A.3.1, para t fixado). Isto implica, segundo o Teorema A.3.3, que $\mathbf{p}(\mathbf{t})$ é IFRA.

Verificamos que $\mathbf{p}(\mathbf{t})$ é NBU.

$$
\sum_{k=3}^{3} p_{k}(t)-\sum_{k=1}^{3} p_{k}(t) \cdot \sum_{k=2}^{3} p_{k}(t)=\frac{0,6+0,6 t^{2}+0,3 t^{4}}{1+0,9 t^{2}+0,3 t^{4}}-1 \cdot \frac{0,9+0,9 t^{2}+0,3 t^{4}}{1+0,9 t^{2}+0,3 t^{4}} \leq 0
$$

e portanto $\mathbf{p}(\mathbf{t})$ é NBU (Definição A.3.3, para t fixado).

Verificaremos que $\mathbf{p}(\mathbf{t})$ é NBUE, ou seja

$$
\sum_{k=j} p_{k}(t) \cdot \mu(t)-\sum_{k=j}^{3} \sum_{l=k}^{3} p_{l}(t) \geq 0
$$

onde

$$
\mu(t)=\sum_{j=1}^{3} \sum_{k=j}^{3} p_{k}(t)=\frac{2,5+1,8 t^{2}+0,9 t^{4}}{1+0,9 t^{2}+0,3 t^{4}}
$$

Concluímos que

$$
\sum_{k=2} p_{k}(t) \cdot \mu(t)-\sum_{k=2}^{3} \sum_{l=k}^{3} p_{l}(t)=\frac{0,75+1,02 t^{2}+0,78 t^{4}+0,36 t^{6}+0,09 t^{8}}{\left(1+0,9 t^{2}+0,3 t^{4}\right)^{2}} \geq 0,
$$

e, portanto, p(t) é NBUE (Definição A.3.4, para t fixado).

Enunciamos o teorema de equivalência das classes de distribuições entre o D-espectro e o Despectro condicional de um network. Nossa motivação agora é verificar que basta estudar as classes 
de distribuições do D-espectro para deduzir as classes para o D-espectro condicional de seu respectivo network. O teorema é enunciado e provado pelo próprio autor.

Teorema 3.2.1 Seja f o D-espectro de um network com n links. Seja $p(t)$ seu D-espectro condicional no instante $t>0$, fixado. Então:

(a) f é IFR (DFR) $\Longleftrightarrow \mathbf{p}(\mathbf{t})$ é IFR (DFR);

(b) f é IFRA (DFRA) $\Longleftrightarrow \mathbf{p}(\mathbf{t})$ é IFRA (DFRA);

(c) $\mathbf{f}$ é NBU $(\mathrm{NWU}) \Longleftrightarrow \mathbf{p}(\mathrm{t})$ é NBU (NWU);

(d) $\mathbf{f}$ é NBUE (NWUE) $\Longleftrightarrow \mathbf{p}(\mathbf{t})$ é NBUE (NWUE).

\section{Prova}

\section{Parte (a)}

Provaremos a equivalência para a classe IFR.

Acondição é suficiente, pois se IFR, ou seja, sob a Definição A.3.1, para todo $k, j=1,2, \ldots, n$ com $\mathrm{k} \geq \mathrm{j}$ e $\mathrm{m}=1,2, \ldots, \mathrm{n}$ fixado

$$
\frac{\sum_{l=k+m+1}^{n} p_{l}(t)}{\sum_{l=k+1}^{n} p_{l}(t)} \leq \frac{\sum_{l=j+m+1}^{n} p_{l}(t)}{\sum_{l=j+1}^{n} p_{l}(t)}
$$

ou equivalentementemente,

$$
\sum_{i=k+m+1}^{n} p_{i}(t) . \sum_{l=j+1}^{n} p_{l}(t)-\sum_{i=k+1}^{n} p_{i}(t) . \sum_{l=j+m+1}^{n} p_{l}(t) \leq 0 .
$$

Tomando o limite quando t tende a zero, temos:

$$
\sum_{i=k+m+1}^{n} f_{i} \cdot \sum_{l=j+1}^{n} f_{l}-\sum_{i=k+1}^{n} f_{i} . \sum_{l=j+m+1}^{n} f_{l} \leq 0 .
$$

Concluímos que, para todo $\mathrm{k}, \mathrm{j}=1,2, \ldots, \mathrm{n}$ com $\mathrm{k} \geq \mathrm{j}$ e $\mathrm{m}=1,2, \ldots, \mathrm{n}$ fixado

$$
\bar{F}_{k+m} \cdot \bar{F}_{j}-\bar{F}_{k} \cdot \bar{F}_{j+m} \leq 0
$$

e, portanto, sob a Definição 3.1.1, f é IFR.

Para verificar a condição necessária da parte (a), suponha, por absurdo, que $\mathbf{p}(\mathbf{t})$ não é IFR, isto é, existem $k_{0}$ e $j_{0}=1, \ldots, n$, com $k_{0} \geq j_{0}$ e $\mathrm{m}=1,2 \ldots, \mathrm{n}$ fixado tais que

$$
\sum_{i=k_{0}+m+1}^{n} p_{i}(t) . \sum_{l=j_{0}+1}^{n} p_{l}(t)-\sum_{i=k_{0}+1}^{n} p_{i}(t) . \sum_{l=j_{0}+m+1}^{n} p_{l}(t)>0 .
$$

Quando t tende a zero,

$$
\sum_{i=k_{0}+m+1}^{n} f_{i} \cdot \sum_{l=j_{0}+1}^{n} f_{l}-\sum_{i=k_{0}+1}^{n} f_{i} . \sum_{l=j_{0}+m+1}^{n} f_{l}>0 .
$$

Então, existem $k_{0}$ e $j_{0}=1, \ldots, n$, com $k_{0} \geq j_{0}$ e $\mathrm{m}=1,2 \ldots, \mathrm{n}$ fixado tais que

$$
\bar{F}_{k_{0}+m} \cdot \bar{F}_{j_{0}}-\bar{F}_{k_{0}} \cdot \bar{F}_{j_{0}+m} \leq 0
$$


Portanto, f não é IFR. O que é uma contradição, pois por hipótese, f é IFR. Para o caso DFR procede-se analogamente.

\section{Parte (b)}

Provemos o caso IFRA.

Suponha inicialmente que $\mathbf{p}(\mathbf{t}), \mathrm{t}>0$, é IFRA, isto é, sob a Definição A.3.2, $\left(\sum_{j=k+1}^{n} p_{j}(t)\right)^{\frac{1}{k}}$ é decrescente em $\mathrm{k}=1,2, \ldots \mathrm{n}$.

Quando t tende a zero, concluímos que $\left(\sum_{j=k+1}^{n} f_{j}\right)^{\frac{1}{k}}=\bar{F}_{k}^{\frac{1}{k}}$ é decrescente em $\mathrm{k}=1,2, \ldots \mathrm{n}$. Portanto, sob a Definição A.3.2, f é IFRA.

Para provar a recíproca, suponha, por absurdo, que $\mathbf{p}(\mathbf{t}), \mathrm{t}>0$, não é IFRA, ou seja

Existe $k_{0}=1, \ldots, \mathrm{n}$ tal que

$$
\left(\sum_{j=k_{0}+1}^{n} p_{j}(t)\right)^{\frac{1}{k_{0}}}<\left(\sum_{j=k_{0}+2}^{n} p_{j}(t)\right)^{\frac{1}{k_{0}+1}} .
$$

Considerando o limite quanto $t$ tende a zero temos

$$
\bar{F}_{k_{0}}^{\frac{1}{k_{0}}}<\bar{F}_{k_{0}+1}^{\frac{1}{k_{0}+1}}
$$

Portanto, f não é IFRA, contradizendo à hipótese de que f é IFRA.

\section{Parte (c)}

Provaremos o caso NBU.

Considere inicialmente que $\mathbf{p}(\mathbf{t})$ é NBU, ou seja, sob a Definição A.3.3, para k e l=1,..,n, e t > 0 fixado.

$$
\sum_{i=k+l+1}^{n} p_{i}(t) \leq \sum_{i=k+1}^{n} p_{i}(t) . \sum_{i=l+1}^{n} p_{i}(t) .
$$

Quando t tende a zero concluímos que $\bar{F}_{k+l} \leq \bar{F}_{k} \cdot \bar{F}_{l}$ e portanto, sob a Definição 3.1.3, f é NBU.

Para verificar a recíproca, suponha, por absurdo, que existem $k_{0}, l_{0}=1,2, \ldots$ n, tais que

$$
\sum_{i=k_{0}+l_{0}+1}^{n} p_{i}(t)>\sum_{i=k_{0}+1}^{n} p_{i}(t) . \sum_{i=l_{0}+1}^{n} p_{i}(t) .
$$

Quando $\mathrm{t}$ tende a zero, existem $k_{0}, l_{0}=1,2, \ldots \mathrm{n}$, tais que $\bar{F}_{k_{0}+l_{0}}>\bar{F}_{k_{0}} \cdot \bar{F}_{l_{0}}$. Portanto, f não é NBU, contradizendo a hipótese de que f é NBU.

\section{Parte (d)}

Provaremos o caso NBUE.

Considere inicialmente que p(t) é NBUE (Definição A.3.4). Então

(i) $\sum_{k=0}^{n} \sum_{j=k+1}^{n} p_{t}(t)<\infty$.

Quando $\mathrm{t}$ tende a zero, $\sum_{k=0}^{n} \bar{F}_{k}<\infty$.

(ii) $\sum_{j=k+1}^{n} p_{j}(t) \cdot \sum_{i=0}^{n} \sum_{l=i+1}^{n} p_{l}(t) \geq \sum_{i=k+1}^{n} \sum_{l=i+1}^{n} p_{l}(t)$. 
Quando $t$ tende a zero, temos que

$$
\bar{F}_{k} \cdot \sum_{i=0}^{n} \bar{F}_{i} \geq \sum_{i=k+1}^{n} \bar{F}_{i}
$$

Portanto, sob a Definição 3.1.4, f é NBUE.

Vamos verificar a recíproca da parte (d) do teorema.

(i) Suponha, por absurdo, que

$$
\sum_{k=0}^{n} \sum_{j=k+1}^{n} p_{t}(t)=\infty
$$

Quando t tende a zero, temos $\sum_{k=0}^{n} \bar{F}_{k}=\infty$. É uma contradição, pois, por hipótese $\sum_{k=0}^{n} \bar{F}_{k}<$ $\infty$.

(ii) Por absurdo, assuma que $\mathbf{p}(\mathbf{t})$ não é NBUE, ou seja, para $\mathrm{t}>0$ fixado, existe $k_{0}=1, \ldots \mathrm{n}$, tal que

$$
\sum_{j=k_{0}+1}^{n} p_{j}(t) \cdot \sum_{i=0}^{n} \sum_{l=i+1}^{n} p_{l}(t)<\sum_{i=k_{0}+1}^{n} \sum_{l=i+1}^{n} p_{l}(t) .
$$

Quando $\mathrm{t}$ tende a zero, concluímos que, para $\mathrm{t}>0$ fixado, existe $k_{0}=1, \ldots, \mathrm{n}$ tal que

$$
\bar{F}_{k_{0}} \cdot \sum_{i=0}^{n} \bar{F}_{i}<\sum_{i=k_{0}+1}^{n} \bar{F}_{i} .
$$

Portanto, f não é NBUE. Uma contradição, pois $\bar{F}_{k}, \mathrm{k}=1, \ldots \mathrm{n}$, é NBUE.

\subsection{Resultados de preservação do D-espectro condicional para o tempo de vida de um network}

Veremos um exemplo de preservação das classes de distribuições do D-espectro condicional para seu respectivo network.

Exemplo 3.3.1 (vide Exemplo A.4.17) Seja T o tempo de vida de um network com Despectro $\mathbf{f}=(0,3 ; 0,3 ; 0,4)$, cujas falhas de seus links, ocorrem de acordo com um Processo de Poisson Não Homogêneo com função de risco $\Lambda(t)=t^{2}$. Seja $\mathbf{p}(\mathbf{t})$ o respectivo D-espectro condicional no instante $\mathrm{t}>0$.

Nosso interesse é analisar a preservação das classes de distribuições do D-espectro condicional $\mathbf{p}(\mathbf{t})$ para o tempo de vida do network.

Seja $\bar{F}=\left(\bar{F}_{0}, \bar{F}_{1}, \bar{F}_{2}, \bar{F}_{3}\right)$, onde $\bar{F}_{k}$ é a função de sobrevivência do D-espectro $\mathbf{f}$ após k falhas de seus links.

A confiabilidade do network no instante t é

$$
\bar{H}(t)=P(T>t)=\sum_{k=0}^{2} \bar{F}_{k} \cdot P(N(t)=k)=e^{-t^{2}}\left(1+0,7 \cdot t^{2}+0,2 \cdot t^{4}\right) .
$$


O k-ésimo componente do D-espectro condicional do network é

$$
p_{k}(t)=\frac{f_{k} \cdot P\left(R_{k}>t\right)}{\bar{H}(t)},
$$

onde $R_{k}$ é o instante da k-ésima falha dos links do network.

Verificaremos que $\mathbf{p}(\mathbf{t})$ tem taxa de falha crescente, isto é (Definição A.3.1), $\frac{p_{k}(t)}{\sum_{j=k}^{n} p_{j}(t)}$ é crescente em $\mathrm{k}=1,2,3,4$. Temos:

$$
\begin{gathered}
p_{1}(t)=\frac{0,3}{1+0,7 \cdot t^{2}+0,2 \cdot t^{4}} \\
p_{2}(t)=\frac{0,3+0,3 \cdot t^{2}}{1+0,7 \cdot t^{2}+0,2 \cdot t^{4}} \\
p_{3}(t)=\frac{0,4+0,4 \cdot t^{2}+0,2 \cdot t^{4}}{1+0,7 \cdot t^{2}+0,2 \cdot t^{4}} .
\end{gathered}
$$

A função de sobrevivência do D-espectro condicional para os valores de k é

$$
\begin{gathered}
\sum_{j=1}^{3} p_{j}(t)=1, \\
\sum_{j=2}^{3} p_{j}(t)=\frac{0,7+0,7 \cdot t^{2}+0,2 \cdot t^{4}}{1+0,7 \cdot t^{2}+0,2 \cdot t^{4}} \\
\sum_{j=3}^{3} p_{j}(t)=\frac{0,4+0,4 \cdot t^{2}+0,2 \cdot t^{4}}{1+0,7 \cdot t^{2}+0,2 \cdot t^{4}} .
\end{gathered}
$$

A primeira igualdade vale, pois o D-espectro condicional é uma distribuição de probabilidades para cada $t>0$, fixado.

As taxas de falhas para os componentes do D-espectro condicional em t são

$$
\begin{gathered}
r_{1}(t)=\frac{p_{1}(t)}{\sum_{j=1}^{3} p_{j}(t)}=\frac{0,3}{1+0,7 . t^{2}+0,2 . t^{4}} \\
r_{2}(t)=\frac{p_{2}(t)}{\sum_{j=2}^{3} p_{j}(t)}=\frac{0,3+0,3 \cdot t^{2}}{0,7+0,7 . t^{2}+0,2 . t^{4}} ; \\
r_{3}(t)=\frac{p_{3}(t)}{\sum_{j=3}^{3} p_{j}(t)}=1 .
\end{gathered}
$$

Observe que $r_{1}(t) \leq r_{2}(t)$, pois $0,3 \leq 0,3+0,3 \cdot t^{2}$ e $1+0,7 \cdot t^{2}+0,2 \cdot t^{4} \geq 0,7+0,7 . t^{2}+0,2 . t^{4}$. É imediato concluir que $r_{2}(t) \leq r_{3}(t)$.

Portanto, o D-espectro condicional $\mathbf{p}(\mathbf{t})$ é IFR.

Usando o Teorema A.3.3, $\mathbf{p}(\mathbf{t})$ também é IFRA, NBU e NBUE.

Observe que

$$
\frac{d^{2} \Lambda(t)}{d t^{2}}=\frac{d^{2} t^{2}}{d t^{2}}=2>0
$$


Portanto, a função de risco $\Lambda(t)=t^{2}$ é côncava.

Note que

$$
\begin{gathered}
\frac{\Lambda(t)}{t}=\frac{t^{2}}{t}=t \\
\frac{d}{d t} \frac{\Lambda(t)}{t}=\frac{d}{d t} \frac{t^{2}}{t}=1>0,
\end{gathered}
$$

isto é, $\frac{\Lambda(t)}{t}$ é crescente em $\mathrm{t}>0$. Portanto, $\Lambda(t)=t^{2}$ é star-shaped (Definição A.3.5).

Observe que $\Lambda(t+x)=(t+x)^{2}=t^{2}+x^{2}+2 . t . x \geq t^{2}+x^{2}=\Lambda(t)+\Lambda(x)$, e, portanto, a função de risco é superaditiva (Definição A.3.6).

Verificamos a logconcavidade de $\bar{H}(t)$. Temos:

$$
\begin{gathered}
\log \bar{H}(t)=-t^{2}+\log \left(1+0,7 t^{2}+0,2 t^{4}\right) ; \\
\frac{d}{d t} \log \bar{H}(t)=\frac{-0,6-1,38 t^{2}-1,5 t^{4}-0,4 t^{6}-0,32 t^{8}}{\left(1+0,7 t^{2}+0,4 t^{4}\right)^{2}} \leq 0 .
\end{gathered}
$$

Sob a Definição A.3.1, concluímos que T é IFR. Usando o Teorema A.3.3, isto implica que T é IFRA, NBU e NBUE.

Com base neste exemplo, percebemos que as classes de distribuições do D-espectro condicional foram preservadas no network, na presença das respectivas propriedades algébricas sobre a função de risco. Isto é provado através do corolário que enunciaremos. Queremos constatar, pela prova deste corolário, que basta estudar as classes de distribuições para o D-espectro condicional e as respectivas propriedades para a função de risco do PPNH para as falhas dos links do network a fim de deduzir as classes de distribuições para o network.

Corolário 3.3.1 Seja $\mathrm{T}$ o tempo de vida um network com $\mathrm{n}$ links, função de distribuição contínua e D-espectro $\mathbf{f}$ com $\mathrm{n}$ links, cujas falhas ocorrem de acordo com um Processo de Poisson Não Homogêneo com função de risco $\Lambda(t)$. Seja $\mathbf{p}(\mathrm{t})$ o respectivo D-espectro condicional e $\{T-t \mid T>t\}$ o respectivo tempo de vida residual no instante t. Então

(a) Se p(t) é IFR (DFR) e $\Lambda(t)$ é côncava, então T é IFR (DFR);

(b) Se $\mathbf{p}(\mathrm{t})$ é IFRA (DFRA) e $\Lambda(t)$ é (anti)star-shaped, então T IFRA (DFRA);

(c) Se $\mathbf{p}(\mathrm{t})$ é NBU (NWU) e $\Lambda(t)$ é superaditiva (subaditiva) então T é NBU (NWU);

(d) Se $\mathbf{p}(\mathrm{t})$ é NBUE (NWUE) e $\Lambda(t)$ é (anti)star-shaped então T é NBUE (NWUE).

Prova Provamos este corolário utilizando o Teorema 3.2.1, seguido dos Teoremas 3.1.2, 3.1.3 e 3.1.4, respectivamente.

A preservação das classes de distribuições do D-espectro condicional para o tempo de vida de networks ocorreu de maneira semelhante à do D-espectro para seu tempo de vida, isto é, quando as falhas dos links ocorrem segundo um Processo de Poisson Não Homogêneo a preservação das classes de distribuições do D-espectro condicional do network para seu tempo de vida depende das respectivas propriedades para sua função de risco. 


\section{Capítulo 4}

\section{Conclusões}

Deduzimos que um network no qual a ordenação das falhas dos links são igualmente prováveis e que ocorrem de acordo com um Processo de Poisson Não Homogêneo as desigualdades estocásticas entre os D-espectros são preservadas nos respectivos tempos de vidas.

Deduzimos também que as classes de distribuições não paramétricas dos D-espectros acompanhadas de propriedades algébricas convenientes da função de risco são preservadas nos tempos de vidas dos networks.

\subsection{Considerações Finais}

Quando o interesse é comparar estocasticamente dois networks mostramos que é suficiente comparar estocasticamente as distribuições dos respectivos D-espectros.

Como na Teoria da Confiabilidade o estudo de classes de distribuições IFR e NBU auxiliam nas tomadas de decisão, na adoção de medidas preventivas e de manutenção. Presume-se que, com os resultados obtidos, o estudo das classes não paramétricas das distribuições discretas dos D-espectros e das propriedades algébricas estudadas para a função de risco sejam suficientes para tais procedimentos.

\subsection{Sugestões para Pesquisas Futuras}

Como pesquisa futura, podemos avaliar resultados de preservações de desigualdades estocásticas e de classes de distribuições para networks e links com mais de dois estados (multiestados) sob independência dos tempos de vidas dos links (Gertsbakh e Shpungin, 2011).

Podemos também estender a ideia de importância de componentes (Barlow e Proschan, 1975) para networks e links multiestados.

É possível estudar a confiabilidade do network utilizando processos pontuais (Bueno, 2015). 
CONCLUSÕES 


\section{Apêndice A}

\section{Apêndice}

\section{A.1 Sistemas coerentes e assinaturas}

O tempo de vida $\mathrm{T}$ de um sistema com n componentes, está relacionado com os tempos de vida de seus componentes $T_{i}$, $\mathrm{i}=1,2, \ldots, \mathrm{n}$, por meio de uma função de estrutura $\Phi$.

$$
T=\Phi\left(T_{1}, T_{2}, \ldots, T_{n}\right)
$$

Definição A.1.1 (Barlow e Proschan (1975)) Considere um sistema com n componentes e $T_{1}, \ldots T_{n}$ seus respectivos tempos de vidas. $\mathrm{O}$ componente $\mathrm{i}, \mathrm{i}=1,2 \ldots, \mathrm{n}$ é relevante para o sistema se $\forall \mathrm{t}, \mathrm{t}>0$, existe uma configuração $\mathbf{X}(\mathrm{t})=\left(X_{1}(t), \ldots, X_{n}(t)\right), \operatorname{com} X_{i}(t)=1_{\left\{T_{i}>t\right\}}$, tais que o sistema funciona se $X_{i}(t)=1$ e falha se $X_{i}(t)=0$.

Não é razoável que a melhora de um componente prejudique o funcionamento do sistema, e que os componentes não sejam essenciais para o funcionamento do sistema. Definimos sistema coerente.

Definição A.1.2 (Barlow e Proschan (1975)) Um sistema $T=\Phi(\mathbf{T})$ é coerente se todo componente é relevante e se $\Phi$ é crescente em cada componente.

Exemplo A.1.1 São sistemas coerentes:

- Sistema em série

$$
T=\min _{1 \leq i \leq n}\left\{T_{i}\right\}
$$

- Sistema em paralelo

$$
T=\max _{1 \leq i \leq n}\left\{T_{i}\right\}
$$

- Sistema série-paralelo

$$
T=\min _{1 \leq j \leq k} \max _{i \in K_{j}}\left\{T_{i}\right\},
$$

onde, $K_{j}$ é o conjunto de cortes minimais, isto é, um conjunto mínimo de componentes cujas falhas causem a falha do sistema (Barlow e Proschan (1975)).

Para avaliar a confiabilidade de um sistema coerente, em termos de seu tempo de vida, a função de estrutura do sistema é uma representação relativamente ampla e de certo modo imprópria. 
Uma função de estrutura útil para o tempo de vida do sistema é através de sua representação por assinatura (Samaniego et al. (2009)), sob a hipótese de que os tempos de vida dos componentes são independentes e identicamente distribuídos (i.i.d.) com distribuição contínua F. Sob tal associação, a assinatura é uma medida estrutural que independe dos tempos de vidas dos componentes e permite uma representação para a confiabilidade do sistema como uma mistura das estatísticas de ordem associadas aos tempos de vida dos componentes útil no cálculo da confiabilidade do sistema e na análise da preservação das desigualdades estocásticas.

As condições da definição de assinatura restringem seu uso generalizado, mas existem várias aplicações em que o uso das assinaturas é apropriado.

- Podemos encontrar alguns exemplos em que a aplicação das assinaturas é apropriada: baterias de sistemas luminosos, pastilhas ou chips de um computador digital, e sistema de velas de ignição automobilísticas;

- As assinaturas de um sistema devem ser usadas cuidadosamente quando se questiona a suposição i.i.d. No entanto, podemos esperar que elas sejam úteis e informativas quando os componentes de um sistema funcionarem sob hipóteses próximas ao caso i.i.d.;

- A assinatura é uma medida estrutural que nos permite comparar realisticamente os sistemas coerentes. Observe que, estocasticamente, um sistema em série com componentes com alta confiabilidade têm um melhor desempenho do que um sistema em paralelo com componentes de baixa confiabilidade.

Definimos assinaturas:

Definição A.1.3 (Samaniego et al., 2009) Seja T o tempo de vida de um sistema coerente com n componentes, com tempos de vida $T_{1}, T_{2}, \ldots, T_{n}$ independentes e identicamente distribuídos com distribuição contínua, definidos em um mesmo espaço de probabilidade $(\Omega, \mathcal{F}, \mathbb{P})$. Seja $\mathrm{T}_{(i)}$, $\mathrm{i}=1,2, \ldots, \mathrm{n}$ a i-ésima estatística de ordem associada aos tempos de vida dos componentes. Então a assinatura do sistema é definida pelo vetor

$$
\mathbf{s}=\left(s_{1}, \ldots, s_{n}\right),
$$

onde, para $\mathrm{i}=1, \ldots, \mathrm{n}$

$$
s_{i}=P\left(T=T_{(i)}\right) .
$$

Nestas suposições pode-se provar que a assinatura não depende diretamente dos tempos de vida dos componentes, mas da estrutura do sistema e da quantidade de componentes que falharam.

Exemplo A.1.2 Considere um sistema com três componentes, do tipo série-paralelo, estruturalmente modelado como mostra a Figura A.1. Suponha que os tempos de vidas dos componentes são i.i.d.

Portanto

$$
\begin{gathered}
s_{1}=P\left(T=T_{(1)}\right)=P\left(T_{1}<T_{2}<T_{3}\right)+P\left(T_{1}<T_{3}<T_{2}\right)=\frac{2}{3 !}=\frac{1}{3} ; \\
s_{2}=P\left(T=T_{(2)}\right)=P\left(T_{2}<T_{1}<T_{3}\right)+P\left(T_{2}<T_{3}<T_{1}\right)+P\left(T_{3}<T_{1}<T_{2}\right)+P\left(T_{3}<T_{2}<T_{1}\right)=\frac{4}{3 !}=\frac{2}{3} ;
\end{gathered}
$$




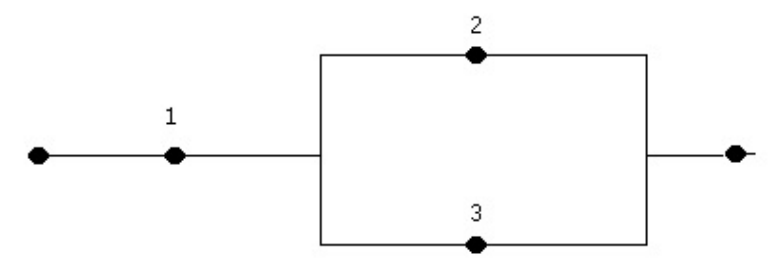

Figura A.1: Figura A.1.1. Sistema tipo série-paralelo com 3 componentes

$$
s_{3}=0
$$

Observe que devido à sua estrutura, o sistema falha até a segunda falha de componentes. Então a assinatura do sistema é: $\mathbf{s}=\left(\frac{1}{3}, \frac{2}{3}, 0\right)$.

Exemplo A.1.3. Em um Sistema k-de-n é necessário que pelo menos k componentes falhem para o sistema falhar. Desse modo, $s_{k}=P\left(T=T_{(k)}\right)=1$, k=1...n e $s_{j}=P\left(T=T_{(j)}\right)=0$, para $\mathrm{j}=1,2, \ldots, \mathrm{k}-1, \mathrm{k}+1, \ldots, \mathrm{n}-1$. A assinatura para um sistema k-de-n é $\mathbf{s}=(0,0,0, \ldots, 0,1,0, \ldots, 0)$.

Observação A.1.1. Em um sistema em série a assinatura do sistema é $\mathbf{s}=(1,0,0, \ldots, 0)$, pois a falha de um componente causa a falha do sistema. Assim, $s_{1}=P\left(T=T_{(1)}\right)=1$ e $s_{k}=P(T=$ $\left.T_{(k)}\right)=0$, para $\mathrm{k}=2, \ldots, \mathrm{n}$. Em um sistema em paralelo, a assinatura do sistema é representada por: $\mathbf{s}=(0,0,0, \ldots, 1)$, pois somente a falha de todos os componentes causa a falha do sistema. Dessa maneira, $s_{n}=P\left(T=T_{(n)}\right)=1$ e $s_{k}=P\left(T=T_{(k)}\right)=0$, para $\mathrm{k}=1,2,3, \ldots, \mathrm{n}-1$.

Definimos a confiabilidade de um sistema coerente.

Definição A.1.4. - disponível no Teorema 1.1 de Samaniego et al. (2009) Seja T o tempo de vida de um sistema coerente com n componentes com tempos de vida $T_{1}, T_{2}, \ldots, T_{n}$ definidos em um mesmo espaço de probabilidade $(\Omega, \mathcal{F}, \mathbb{P})$. A confiabilidade do sistema no instante $\mathrm{t}, \mathrm{t}>0$, fixado, é definida como a probabilidade de sobreviver ao instante t, $P(T>t)$.

Sob a hipótese dos componentes independentes, identicamente distribuídos e com distribuição contínua, utilizando a Lei da Probabilidade Total, podemos representar a confiabilidade do sistema no instante $t>0$, através da mistura

$$
\begin{gathered}
P(T>t)=\sum_{i=1}^{n} P\left(T>t, T=T_{(i)}\right)=\sum_{i=1}^{n} P\left(T>t \mid T=T_{(i)}\right) P\left(T=T_{(i)}\right) \\
=\sum_{i=1}^{n} P\left(T_{(i)}>t \mid T=T_{(i)}\right) P\left(T=T_{(i)}\right)=\sum_{i=1}^{n} P\left(T_{(i)}>t\right) P\left(T=T_{(i)}\right)=\sum_{i=1}^{n} s_{i} . P\left(T_{(i)}>t\right) .
\end{gathered}
$$

A penúltima igualdade é válida, pois os eventos $\left\{T_{(i)}>t\right\}$ e $\left\{T=T_{(i)}\right\}$ são independentes, desde que o primeiro evento depende da distribuição $\mathrm{F}$, enquanto que o segundo evento depende apenas da ordenação dos tempos de vida dos componentes. Tal argumento é provado no Lema A.1.1.

Enunciamos um lema em que um evento que depende apenas da ordem das falhas dos componentes independe de um evento que depende apenas da ordenação de tempos de vidas dos componentes que nos auxilia na construção da confiabilidade de sistemas como mistura das confiabilidades dos instantes de falhas (vide seção 2.1). 
Lema A.1.1 (Lema 8.3.11 de Randles e Wolfe (1979)) Sejam $Z_{1}, \ldots Z_{n}$ v.a. i.i.d. contínuas com função densidade de probabilidade $f$ com a função densidade conjunta $f_{\mathbf{Z}}$ para $\mathbf{Z}=\left(Z_{1}, \ldots, Z_{n}\right)$. Seja $\mathbf{R}^{*}$ o vetor dos postos (ordens) associados a essas observações e $Z_{(1)}, \ldots Z_{(n)}$ as estatísticas de ordem associadas às variáveis $Z_{1}, \ldots Z_{n}$. Então, $\mathbf{R}^{*}$ e $Z_{(1)}, \ldots Z_{(n)}$ são independentes.

Prova Seja g a f.d.p. de $Z_{(1)}, \ldots Z_{(n)}$ dado $\mathbf{R}^{*}=\mathbf{r}^{*}$. Considere h a f.d.p. conjunta de $Z_{(1)}, \ldots Z_{(n)}$ e $\mathbf{R}^{*}=\mathbf{r}^{*}$. Temos:

$$
g\left(z_{(1)}, \ldots z_{(n)} \mid \mathbf{R}^{*}=\mathbf{r}^{*}\right)=\frac{h\left(z_{(1)}, \ldots z_{(n)}, \mathbf{R}^{*}=\mathbf{r}^{*}\right)}{P\left(\mathbf{R}^{*}=\mathbf{r}^{*}\right)} .
$$

Mas $P\left(\mathbf{R}^{*}=\mathbf{r}^{*}\right)=\frac{1}{n !}$, para todo vetor $\mathbf{r}^{*}$. Assim, dizemos que a distribuição conjunta das estatísticas de ordem é permutável, ou seja, para todo $\mathbf{r}^{*}$ e $\mathbf{r}^{\prime}$ vetores de postos associados às estatísticas de ordem. Temos

$$
P\left(\mathbf{R}^{*}=\mathbf{r}^{*}\right)=P\left(\mathbf{R}^{*}=\mathbf{r}^{\prime}\right)=\frac{1}{n !} .
$$

Dessa maneira, para $\left\{\mathbf{R}^{*}=\mathbf{r}^{*}\right\}$, podemos escolher um vetor de postos $\mathrm{r}^{*}=(1,2, \ldots, \mathrm{n})$ como argumento similar a um outro vetor $\mathrm{r}^{\prime}=\left(\pi_{1}, \ldots, \pi_{n}\right)$, onde $\pi_{k}$ é um k-ésimo posto de uma observação após uma nova ordenação $\pi$ das observações. Ademais, na escolha $\mathrm{r}^{*}=(1,2, \ldots, \mathrm{n})$ para o vetor de postos, as estatísticas de ordem terão seus postos coincidindo com os valores do vetor $\mathrm{r}^{*}$, após ordenarmos as observações $Z_{i}, \mathrm{i}=1,2, \ldots, \mathrm{n}$. Então:

$$
g\left(z_{(1)}, \ldots z_{(n)} \mid \mathbf{R}^{*}=\mathbf{r}^{*}\right)=\frac{f_{\mathbf{Z}}\left(z_{(1)}, \ldots z_{(n)}\right)}{P\left(\mathbf{R}^{*}=\mathbf{r}^{*}\right)} .
$$

Como $Z_{k}, k=1,2, \ldots, n$ são independentes e identicamente distribuídas, temos:

$$
g\left(z_{(1)}, \ldots z_{(n)} \mid \mathbf{R}^{*}=\mathbf{r}^{*}\right)=\frac{\prod_{k=1}^{n} f\left(z_{(k)}\right)}{\frac{1}{n !}}=n ! \prod_{k=1}^{n} f\left(z_{(k)}\right)=g_{\mathbf{Z}}(\mathbf{z})
$$

que é a função densidade conjunta de estatísticas de ordem associadas a variáveis aleatórias independentes e identicamente distribuídas.

Ao particularizar a demonstração para o caso univariado, para uma das estatísticas de ordem, dados os postos das variáveis temos:

$$
\begin{aligned}
g\left(z_{(k)} \mid \mathbf{R}^{*}=\right. & \left.\mathbf{r}^{*}\right)=\int_{0}^{\infty} \ldots \int_{0}^{\infty} g\left(z_{(1)}, \ldots z_{(n)} \mid \mathbf{R}^{*}=\mathbf{r}^{*}\right) d z_{(1)} d z_{(2)} \ldots d z_{(k-1)} d z_{(k+1)}, \ldots d z_{(n)} \\
& =\int_{0}^{\infty} \ldots \int_{0}^{\infty} g_{\mathbf{Z}}(\mathbf{z}) d z_{(1)} d z_{(2)} \ldots d z_{(k-1)} d z_{(k+1)}, \ldots d z_{(n)}=g\left(z_{(k)}\right) .
\end{aligned}
$$

Com essa demonstração fica provada a independência entre um evento que dependa do posto do instante de uma k-ésima falha, com $\mathrm{k}=1, \ldots, \mathrm{n}$, em relação a um evento que dependa da permutação entre os postos dos instantes de falha, e portanto, entre os componentes. 


\section{A.2 Desigualdades estocásticas}

As desigualdades estocásticas mais utilizadas são definidas:

Definição A.2.1 - Seção 1.A de Shaked e Shanthikumar (2007) Sejam S e T dois tempos de vidas. Dizemos que $\mathrm{S}$ é estocasticamente menor que $\mathrm{T}\left(\mathrm{S} \leq_{S T} \mathrm{~T}\right)$ se, para todo $t>0: P(S>$ $t) \leq P(T>t)$.

Se $\mathrm{S}$ e $\mathrm{T}$ admitem funções discretas de probabilidade, $\mathrm{S} \leq_{S T} \mathrm{~T}$ se, e somente se, para $\mathrm{k} \in \mathbb{N}$ : $P(S>k) \leq P(T>k)$.

Estas desigualdades sugerem que um objeto com tempo de vida T é mais confiável do que com tempo de vida $\mathrm{S}$.

Definição A.2.2 - Seção 1.B de Shaked e Shanthikumar (2007) Sejam S e T dois tempos de vida. Dizemos que $\mathrm{S}$ é menor, em taxa de falha (hazard rate), do que $\mathrm{T}\left(\mathrm{S} \leq_{H R} \mathrm{~T}\right.$ ) se, para todo $t>0: \frac{P(T>t)}{P(S>t)}$ é crescente em $\mathrm{t}$. Equivalentemente, se $\mathrm{S}$ e $\mathrm{T}$ admitem funções densidades de probabilidade, $\mathrm{S} \leq_{H R} \mathrm{~T}$ se, e somente se, $\lambda_{S} \geq \lambda_{T}$, onde $\lambda_{S}$ e $\lambda_{T}$ são as taxas de falha de $\mathrm{S}$ e de $\mathrm{T}$ respectivamente.

Alternativamente, se $\mathrm{S}$ e T admitem funç oes discretas de probabilidade, $\mathrm{S} \leq_{H R} \mathrm{~T}$ se, e somente se, $\in \mathbb{N}: \frac{P(T>k)}{P(S>k)}$, é crescente em $\mathrm{k} \in \mathbb{N}$. Equivalentemente, $\mathrm{S} \leq_{H R} \mathrm{~T}$ se, e somente se, para todo $\mathrm{k}$ $\in \mathbb{N}: \frac{P(S=k)}{P(S \geq k)} \geq \frac{P(T=k)}{P(T \geq k)}$.

Interpretamos que um objeto com tempo de vida $\mathrm{S}$ tem maior propensão a falhas do que um objeto com tempo de vida T.

Definição A.2.3 - Seção 1.C de Shaked e Shanthikumar (2007) Sejam S e T dois tempos de vidas.

Se S e T admitem funções densidades de probabilidades, dizemos que $\mathrm{S}$ é menor, em razão de verossimilhanças, do que $\mathrm{T}\left(\mathrm{S} \leq_{L R} \mathrm{~T}\right)$ se, para todo $\mathrm{t} \in \mathbb{R}: \frac{g_{T}(t)}{g_{S}(t)}$ é crescente em $\mathrm{t}$, onde $g_{T}$ e $g_{S}$ são as funções densidades de probabilidade de $\mathrm{T}$ e $\mathrm{S}$ respectivamente.

Se S e T admitem funções discretas de probabilidade, então $\mathrm{S} \leq_{L R} \mathrm{~T}$ se, e somente se, para todo $\mathrm{k} \in \mathbb{N}: \frac{P(T=k)}{P(S=k)}$ é crescente em k.

Existe uma hierararquia entre as desigualdades estocásticas. Observamos que a ordenação através da razão de verossimilhanças monótona implica a ordenação através das taxas de falhas, que, por sua vez, implica a ordenação estocástica.

Teorema A.2.1 (ver Teoremas 1.B.1 e 1.C.1 de Shaked e Shanthikumar (2007)) Sejam $\mathrm{S}$ e $\mathrm{T}$ duas variáveis aleatórias definidas em um mesmo espaço de probabilidade $(\Omega, \mathcal{F}, \mathbb{P})$. Então

$$
S \leq_{H R} T \Rightarrow S \leq_{S T} T \text {. }
$$

Se S e T têm funções de distribuições absolutamente contínuas (ou discretas), então

$$
S \leq_{L R} T \Rightarrow S \leq_{H R} T .
$$

Prova: Sejam f, g as funções densidades (ou discretas) de probabilidade de $\mathrm{S}$ e $\mathrm{T}$ respectivamente. Sejam $\bar{F}$ e $\bar{G}$ suas funções de sobrevivência. Então 


$$
\begin{gathered}
S \leq_{H R} T \Rightarrow \frac{\bar{G}(u)}{\bar{F}(u)} \geq \frac{\bar{G}(v)}{\bar{F}(v)}, u \geq v \Rightarrow \frac{\bar{G}(u)}{\bar{F}(u)} \geq \frac{\bar{G}(0)}{\bar{F}(0)}, u \geq 0 \\
\Rightarrow \frac{\bar{G}(u)}{\bar{F}(u)} \geq 1, \Rightarrow \bar{G}(u) \geq \bar{F}(u) \Rightarrow S \leq_{S T} T . \\
S \leq_{L R} T \Rightarrow \frac{g(s)}{f(s)} \leq \frac{g(t)}{f(t)}, s \leq t \\
g(t) f(s)-f(t) g(s) \geq 0 \Rightarrow \int_{u}^{\infty} \int_{v}^{\infty}[d G(t) d F(s)-d F(t) d G(s)] \geq 0, \quad u \geq v \\
\Rightarrow \int_{u}^{\infty} d G(t) \int_{v}^{\infty} d F(s)-\int_{u}^{\infty} d F(t) \int_{v}^{\infty} d G(s) \geq 0, \quad u \geq v \\
\Rightarrow \bar{G}(u) \bar{F}(v)-\bar{G}(v) \bar{F}(u) \geq 0, u \geq v \Rightarrow \frac{\bar{G}(u)}{\bar{F}(u)} \geq \frac{\bar{G}(v)}{\bar{F}(v)}, \quad u \geq v \Rightarrow S \leq_{H R} T .
\end{gathered}
$$

As recíprocas das implicações acima nem sempre são válidas. Eis dois contra-exemplos:

Exemplo A.2.1 Considere $\mathbf{f}$ e $\mathbf{g}$ duas funções discretas de probabilidade, tais que $\mathbf{f}=\left(\frac{1}{2}, \frac{1}{3}, \frac{1}{6}\right)$ e $\mathbf{g}=\left(\frac{1}{3}, \frac{1}{3}, \frac{1}{3}\right)$.

As respectivas funções de sobrevivência são: $\overline{\mathbf{F}}=\left(1, \frac{1}{2}, \frac{1}{6}\right)$ e $\overline{\mathbf{G}}=\left(1, \frac{2}{3}, \frac{1}{3}\right)$.

Concluímos que $\overline{\mathbf{F}} \leq \overline{\mathbf{G}}$ e, portanto, $\mathbf{f} \leq_{S T} \mathbf{g}$.

Vamos avaliar a desigualdade através das taxas de falhas, usando a relação $\frac{\bar{G}}{\bar{F}}$. Temos:

$\frac{\bar{G}(1)}{\bar{F}(1)}=1, \frac{\bar{G}(2)}{\bar{F}(2)}=\frac{\frac{2}{3}}{\frac{1}{3}}=2$, e $\frac{\bar{G}(3)}{\bar{F}(3)}=\frac{\frac{1}{6}}{\frac{1}{3}}=\frac{1}{2}$.

Então $\frac{\bar{G}}{F}$ é não-crescente, e $\mathbf{f} \not_{H R} \mathbf{g}$. Portanto $\mathbf{f} \leq_{S T} \mathbf{g} \nRightarrow \mathbf{f} \leq_{H R} \mathbf{g}$.

Exemplo A.2.2 Considere $\mathbf{f}$ e $\mathbf{g}$ duas funções discretas de probabilidade, tais que $\mathbf{f}=(0,2 ; 0,2 ; 0,6)$ e $\mathbf{g}=(0,3 ; 0,4 ; 0,3)$.

As respectivas funções de sobrevivência são: $\overline{\mathbf{F}}=(1 ; 0,7 ; 0,3)$ e $\overline{\mathbf{G}}=(1 ; 0,8 ; 0,6)$.

Vamos avaliar a desigualdade através das taxas de falhas. Temos:

$\frac{\bar{G}(1)}{\bar{F}(1)}=1, \frac{\bar{G}(2)}{\bar{F}(2)}=\frac{0,8}{0,7}=\frac{8}{7}$, e $\frac{\bar{G}(3)}{\bar{F}(3)}=\frac{0,6}{0,3}=2$.

Então $\frac{\bar{G}}{\bar{F}}$ é crescente, e $\mathbf{f} \leq_{H R} \mathbf{g}$.

Avaliamos a desigualdade através da razão de verossimilhanças:

$\frac{g(1)}{f(1)}=\frac{0,2}{0,3}=\frac{2}{3}, \frac{g(2)}{f(2)}=\frac{0,2}{0,4}=0,5$, e $\frac{g(3)}{f(3)}=\frac{0,6}{0,3}=2$.

Então $\frac{g}{f}$ é não-crescente, e $\mathbf{f} \not_{L R} \mathbf{g}$. Portanto $\mathbf{f} \leq_{H R} \mathbf{g} \nRightarrow \mathbf{f} \leq_{L R} \mathbf{g}$.

Definição A.2.4. Sejam A e B dois subconjuntos na reta real. Uma função não-negativa $K$ definida em A x B chama-se totalmente positiva de ordem $2\left(T P_{2}\right)$ (Karlin, 1968), se para $a_{1}<a_{2}$ e $b_{1}<b_{2}$, com $a_{1}, a_{2} \in A$ e $b_{1}, b_{2} \in B$,

$$
K\left(a_{1}, b_{1}\right) K\left(a_{2}, b_{2}\right)-K\left(a_{1}, b_{2}\right) K\left(a_{2}, b_{1}\right) \geq 0 .
$$

Isto equivale a

$$
\frac{K\left(a_{1}, b_{1}\right)}{K\left(a_{1}, b_{2}\right)} \leq \frac{K\left(a_{2}, b_{1}\right)}{K\left(a_{2}, b_{2}\right)}
$$


Exemplo A.2.3. Considere $\{N(t): t \geq 0\}$ um Processo de Poisson Não Homogêneo, com função de risco $\Lambda(t), \mathrm{t}>0$. Para $0 \leq s \leq t$ e $\mathrm{j} \leq \mathrm{k}=0,1,2, \ldots$, temos

$$
\begin{gathered}
P(N(t)=k) \cdot P(N(s)=j)-P(N(t)=j) \cdot P(N(s)=k) \\
=\frac{e^{-\Lambda(t)} \cdot \Lambda(t)^{k}}{k !} \cdot \frac{e^{-\Lambda(s)} \cdot \Lambda(s)^{j}}{j !}-\frac{e^{-\Lambda(t)} \cdot \Lambda(t)^{j}}{j !} \cdot \frac{e^{-\Lambda(s)} \cdot \Lambda(s)^{k}}{k !} \\
=\frac{e^{-\Lambda(t)-\Lambda(s)}}{k ! j !} \cdot\left[\Lambda(t)^{k} \cdot \Lambda(s)^{j}-\Lambda(t)^{j} \cdot \Lambda(s)^{k}\right] .
\end{gathered}
$$

Basta verificar que $\Lambda(t)^{k} . \Lambda(s)^{j}-\Lambda(t)^{j} \cdot \Lambda(s)^{k} \geq 0$.

Como $\Lambda(t)$ é crescente e $\mathrm{k} \geq \mathrm{j}=0,1,2 \ldots$, temos que

$$
\left[\frac{\Lambda(t)}{\Lambda(s)}\right]^{k} \geq\left[\frac{\Lambda(t)}{\Lambda(s)}\right]^{j} \Rightarrow \Lambda(t)^{k} \cdot \Lambda(s)^{j}-\Lambda(t)^{j} \cdot \Lambda(s)^{k} \geq 0
$$

Então

$$
P(N(t)=k) \cdot P(N(s)=j)-P(N(t)=j) \cdot P(N(s)=k) \geq 0,
$$

e portanto, $P(N(t)=k)$ é $\mathrm{TP}_{2}$ em t $>0$ e $\mathrm{k}=0,1,2, \ldots$ para um Processo de Poisson Não Homogêneo com função de risco $\Lambda(t)$.

Teorema A.2.2 (ver Teoremas 1.A.6, 1.B.14 e 1.C.17 de Shaked e Shanthikumar (2007)) Seja $\mathcal{X}$ um subconjunto de $\mathbb{R}$ e $\left\{G_{\theta}, \theta \in \mathcal{X}\right\}$ ums família de distribuições. Sejam $\Theta_{1}$ e $\Theta_{2}$ duas variáveis aleatórias com suportes em $\mathcal{X}$ com respectivas funções de distribuições $F_{1}$ e $F_{2}$. Sejam $Y_{1}$ e $Y_{2}$ duas variáveis aleatórias tais que $Y_{i}={ }_{S T} X\left(\Theta_{i}\right) \mathrm{i}=1,2$, onde $X\left(\Theta_{i}\right)$ é uma variável aleatória cuja função de distribuição é

$$
H(y)=\int_{\mathcal{X}} G_{\theta}(y) d F_{i}(\theta)
$$

com funccão densidade de probabilidade

$$
h(y)=\int_{\mathcal{X}} g_{\theta}(y) d F_{i}(\theta)
$$

Seja $\bar{H}$ sua respectiva função de sobrevivência, dada por

$$
\bar{H}(y)=\int_{\mathcal{X}} \bar{G}_{\theta}(y) d F_{i}(\theta)
$$

para $y \in \mathbb{R}, \mathrm{i}=1,2$

a) Se $X(\theta) \leq_{S T} X\left(\theta^{\prime}\right)$, para $\theta \leq \theta^{\prime}$ e $\Theta_{1} \leq_{S T} \Theta_{2}$, então $Y_{1} \leq_{S T} Y_{2}$;

b) Se $X(\theta) \leq_{H R} X\left(\theta^{\prime}\right)$, para $\theta \leq \theta^{\prime}$ e $\Theta_{1} \leq_{H R} \Theta_{2}$, então $Y_{1} \leq_{H R} Y_{2}$;

c) Se $X(\theta) \leq_{L R} X\left(\theta^{\prime}\right)$, para $\theta \leq \theta^{\prime}$ e $\Theta_{1} \leq_{L R} \Theta_{2}$, então $Y_{1} \leq_{L R} Y_{2}$.

\section{Prova}

a) $X(\theta) \leq_{S T} X\left(\theta^{\prime}\right)$ implica $P(X(\theta)>y)$ é crescente em $\theta$ para todo y. Então

$$
P\left(Y_{1}>y\right)=\int_{\mathcal{X}} P(X(\theta)>y) d F_{1}(\theta) \leq \int_{\mathcal{X}} P(X(\theta)>y) d F_{2}(\theta)=P\left(Y_{2}>y\right) .
$$


A desigualdade vale pois $\Theta_{1} \leq_{S T} \Theta_{2}$ e $P\left(Y_{i}>y\right)$ é a esperança de uma função crescente em $\theta \in \mathcal{X}$.

Portanto $Y_{1} \leq_{S T} Y_{2}$.

b) $X(\theta) \leq_{H R} X\left(\theta^{\prime}\right)$ implica $P(X(\theta)>y)$ é $T P_{2}$ em $\theta \in \mathcal{X}$ e $y \in \mathbb{R}$. $\Theta_{1} \leq_{H R} \Theta_{2}$, istoé, $\bar{F}_{i}(\theta)$ $\mathrm{TP}_{2}$ em $\mathrm{i}=1,2$ e $\theta \in \mathcal{X}$.

Além disso $X(\theta) \leq_{H R} X\left(\theta^{\prime}\right)$ implica $X(\theta) \leq_{S T} X\left(\theta^{\prime}\right)$, ou seja, $P(X(\theta)>y)$ é crescente em $\theta>0$.

Assim, pelo Teorema 2.1 de Joag-Dev et al. (1992), $\bar{H}_{i}(y)$ é $\mathrm{TP}_{2}$ em i=1,2 e $y \in \mathbb{R}$ e $Y_{1} \leq_{H R} Y_{2}$.

c) $X(\theta) \leq_{L R} X\left(\theta^{\prime}\right)$ implica $g_{\theta}(y)$ é $T P_{2}$ em $\theta \in \mathcal{X}$ e $y \in \mathbb{R} . \Theta_{1} \leq_{L R} \Theta_{2}$ implica $\bar{f}_{i}(\theta) \mathrm{TP}_{2}$ em $\mathrm{i}=1,2$ e $\theta \in \mathcal{X}$. Além disso $X(\theta) \leq_{L R} X\left(\theta^{\prime}\right)$ implica $X(\theta) \leq_{H R} X\left(\theta^{\prime}\right)$, ou seja, $P(X(\theta)>y)$ é $\mathrm{TP}_{2}$ em $\theta>0$.

Portanto para $0 \leq y_{1} \leq y_{2}$

$$
\iint_{\theta \leq \theta^{\prime}}\left(g_{\theta}\left(y_{1}\right) \cdot g_{\theta}\left(y_{2}\right)-g_{\theta}\left(y_{2}\right) \cdot g_{\theta}\left(y_{1}\right)\right) \cdot\left(d F_{1} \theta \cdot d F_{2} \theta^{\prime}-d F_{1} \theta^{\prime} \cdot d F_{2} \theta\right) \geq 0
$$

e

$$
\iint_{\theta>\theta^{\prime}}\left(g_{\theta}\left(y_{1}\right) \cdot g_{\theta}\left(y_{2}\right)-g_{\theta}\left(y_{2}\right) \cdot g_{\theta}\left(y_{1}\right)\right) \cdot\left(d F_{1} \theta \cdot d F_{2} \theta^{\prime}-d F_{1} \theta^{\prime} \cdot d F_{2} \theta\right) \geq 0 .
$$

Ambas as integrais são não-negativas devido à integração de produto de expressões de mesmo sinal. Temos:

$$
\begin{gathered}
\iint_{\mathcal{X}}\left(g_{\theta}\left(y_{1}\right) \cdot g_{\theta}\left(y_{2}\right)-g_{\theta}\left(y_{2}\right) \cdot g_{\theta}\left(y_{1}\right)\right) \cdot\left(d F_{1} \theta \cdot d F_{2} \theta^{\prime}-d F_{1} \theta^{\prime} \cdot d F_{2} \theta\right) \geq 0 \\
\Longleftrightarrow g_{1}\left(y_{1}\right) \cdot g_{2}\left(y_{2}\right)-g_{1}\left(y_{2}\right) \cdot g_{2}\left(y_{1}\right)-g_{1}\left(y_{2}\right) \cdot g_{2}\left(y_{1}\right)+g_{1}\left(y_{1}\right) \cdot g_{2}\left(y_{2}\right) \geq 0 \\
\Longleftrightarrow 2 \cdot g_{1}\left(y_{1}\right) \cdot g_{2}\left(y_{2}\right)-2 \cdot g_{1}\left(y_{2}\right) \cdot g_{2}\left(y_{1}\right) \geq 0 \Longleftrightarrow g_{1}\left(y_{1}\right) \cdot g_{2}\left(y_{2}\right)-g_{1}\left(y_{2}\right) \cdot g_{2}\left(y_{1}\right) \geq 0 .
\end{gathered}
$$

Concluímos que $g_{i}(y)$ é $T P_{2}$ em i=1...n e y $>0$ e portanto $Y_{1} \leq_{L R} Y_{2}$.

\section{A.3 Classes de distribuições}

Inicialmente enunciamos uma propriedade que nos auxilia a provar a preservação de algumas classes de distribuição do D-espectro de um network para o respectivo tempo de vida.

Propriedade da diminuição da variação (Karlin, 1968)

(a) Versão usual para variáveis aleatórias com distribuições contínuas Sejam $f$ e $g$ duas funções reais, tais que $f: \mathcal{B}\left(\mathbb{R}^{2}\right) \rightarrow \mathbb{R}$ e $g: \mathcal{B}(\mathbb{R}) \rightarrow \mathbb{R}$.

Seja h tal que

$$
h(x)=\int_{\mathbb{R}} f(x, z) \cdot g(z) d z .
$$

Se fé $T P_{2}$ em $\mathbb{R} \times \mathbb{R}$ e $\mathrm{g}(\mathrm{t})$ tem no máximo $\mathrm{r}$ trocas de sinais em $\mathrm{t} \in \mathbb{R}$, então $\mathrm{h}$ tem no máximo $\mathrm{r}-1$ trocas de sinais em $\mathrm{t} \in \mathbb{R}$. 
Se h e g têm a mesma quantidade de trocas de sinais, então ocorrem no mesmo sentido.

(b) Versão usual para variáveis aleatórias com distribuições discretas Sejam f e g duas funções discretas, tais que $f: \mathbb{N} \times \mathbb{R} \rightarrow \mathbb{R}$ e $g: \mathbb{N} \rightarrow \mathbb{R}$. Seja h tal que

$$
h(t)=\sum_{k=0}^{\infty} f(k, t) \cdot g(k) .
$$

Se fé $T P_{2}$ em $\mathbb{N} \times \mathbb{N}$ e $\mathrm{g}(\mathrm{k})$ tem no máximo $\mathrm{r}$ trocas de sinais em $\mathrm{k} \in \mathbb{N}$, então $\mathrm{h}$ tem no máximo $\mathrm{r}-1$ trocas de sinais em $\mathrm{t} \in \mathbb{R}$.

Se $\mathrm{h}$ e g têm a mesma quantidade de trocas de sinais, em $\mathrm{t} \in \mathbb{R}$ e em $\mathrm{k} \in \mathbb{N}$ respectivamente, então ocorrem no mesmo sentido.

Apresentamos as classes de distribuições para tempos de vidas com distribuições contínuas, definidas em $(\Omega, \mathcal{F}, P)$.

Definição A.3.1 Considere T um tempo de vida com distribuição contínua $\mathrm{F}$ em $(0, \infty)$. Dizemos que $\mathrm{T}$ tem taxa de falha crescente (IFR) se, para $\mathrm{x}>0, \frac{\bar{F}(t+x)}{\bar{F}(t)}$ é decrescente em $\mathrm{t}>0$.

Equivalentemente, se T tem função densidade de probabilidade $\mathrm{f}(\mathrm{t})$, T é IFR se $\frac{f(t)}{\bar{F}(t)}$ é crescente em t.

Observação A.3.1 T tem taxa de falha decrescente (DFR) se na definição acima as monotonicidades são contrárias.

Definição A.3.2 Seja $T$ um tempo de vida com distribuição contínua F. Dizemos que $T$ tem taxa de falha crescente em média (IFRA) se $-\frac{1}{t} \cdot \log \bar{F}(t)$ é crescente em $\mathrm{t}>0$.

Equivalentemente, T possui a propriedade IFRA se $\bar{F}(t)^{\frac{1}{t}}$ é decrescente em $\mathrm{t}>0$.

Observação A.3.2 T tem taxa de falha decrescente em média (DFRA) se as monotonicidades da definição acima são contrárias.

Definição A.3.3 Seja T um tempo de vida com função de distribuição contínua F. Dizemos que $\mathrm{T}$ é novo melhor que usado (NBU) se, para $\mathrm{x}>0$ e $\mathrm{t}>0$.

$$
\bar{F}(x+t) \leq \bar{F}(x) \bar{F}(t) .
$$

Observação A.3.3 T é novo pior que usado (NWU) se a desigualdade na definição acima é contrária.

Definição A.3.4 Seja T um tempo de vida com função de distribuição contínua F. Dizemos que $\mathrm{T}$ é novo melhor que usado em média (NBUE), para $\mathrm{t}>0$ se

(i) $\mu=E(T)<\infty$;

(ii) $\bar{F}(t) \cdot \mu \geq(\leq) \int_{t}^{\infty} \bar{F}(x) d x$.

Observação A.3.4 T é novo pior que usado em média (NWUE) se a desigualdade na definição acima é contrária. 
Definição A.3.5 Seja g uma função real. g é star-shaped (antistar-shaped) se, e somente se, $\frac{g(x)}{x}$ é crescente (decrescente) em $\mathrm{x}>0$.

Definição A.3.6 Considere f uma funç ao real. f é superaditiva (subaditiva) em algum subconjunto $\mathrm{A}$ de $\mathbb{R}$, se, para todo $\mathrm{x}, \mathrm{y} \in \mathrm{A}$

$$
f(x+y) \geq(\leq) f(x)+f(y) .
$$

Observe que se uma função de sobrevivência $\bar{H}$ é NBU, isto é, $\bar{H}(x+y) \leq \bar{H}(x) \bar{H}(y)$ em x > 0 e y $>0, \bar{H}(x)$ é subaditiva.

Definição A.3.9 Seja A um subconjunto de $\mathbb{R}$ e $\mathrm{x} \in \mathrm{A}$. Denominamos a função $\operatorname{Ei}(x)=\int_{-x}^{\infty} \frac{e^{z}}{z} d z$ de Função Exponencial Integral em x.

Teorema A.3.1 - Teorema 2.17 de Barlow e Proschan (1975) Seja T um tempo de vida com distribuição contínua F. Então T é IFRA se, e somente se, $-\log \bar{F}(t)$ é star-shaped.

Teorema A.3.2 - Teorema 2.18 de Barlow e Proschan (1975) Seja T um tempo de vida com distribuição contínua F. Então, T é IFRA (DFRA) se, e somente se, para $\lambda>0, \bar{F}(t)-e^{-\lambda . t}$ tem uma troca de sinal de positivo para negativo (negativo para positivo) em relação a $t>0$.

Assim como as desigualdades estocásticas as classes de distribuições apresentam entre si uma hierarquia, provada neste teorema.

Teorema A.3.3 - Hierarquia das classes de distribuições (Barlow e Proschan, 1975) Seja T um tempo de vida com função de distribuição contínua (discreta) F e função densidade (discreta) de probabilidade $\mathrm{f}$.

(i) Se T é IFR (DFR), então T é IFRA (DFRA);

(ii) Se T é IFRA (DFRA), então T é NBU (NWU);

(iii) Se T é NBU (NWU), então T é NBUE (NWUE).

Prova Consideramos as provas para T com função de distribuição absolutamente contínua.

(i) Provamos a partir da classe IFR.

Verificamos que $\mathrm{R}(\mathrm{t})$ é star-shaped, onde $\mathrm{R}(\mathrm{t})$ é a função de risco do tempo de vida $\mathrm{T}$ em $\mathrm{t}>0$, com

$$
R(t)=\int_{0}^{t} r(s) d s=-\log \bar{F}(t) .
$$

A taxa de falha de $\mathrm{T}$ no instante $\mathrm{t}$ é

$$
r(t)=\frac{f(t)}{\bar{F}(t)}
$$

Como T é IFR, para $0 \leq s \leq t$, temos que $r(t) \geq r(s)$.

Se $0 \leq s^{\prime} \leq t^{\prime}$, então

$$
\int_{0}^{t^{\prime}} r(t) d t \geq \int_{0}^{t^{\prime}} r(s) d t \Rightarrow R\left(t^{\prime}\right) \geq t^{\prime} \cdot r(s) \Rightarrow \int_{0}^{s^{\prime}} R\left(t^{\prime}\right) d s \geq \int_{0}^{s^{\prime}} t^{\prime} \cdot r(s) d s \Rightarrow s^{\prime} \cdot R\left(t^{\prime}\right) \geq t^{\prime} \cdot R\left(s^{\prime}\right)
$$




$$
\Rightarrow \frac{R\left(t^{\prime}\right)}{t^{\prime}} \geq \frac{R\left(s^{\prime}\right)}{s^{\prime}} \Rightarrow \frac{-\log \bar{F}\left(t^{\prime}\right)}{t^{\prime}} \geq \frac{-\log \bar{F}\left(s^{\prime}\right)}{s^{\prime}} .
$$

Concluímos que R é star-shaped, portanto T é IFRA.

(ii) Provamos a partir da classe IFRA

Verificaremos se, para t e $\mathrm{x}>0$

$$
\bar{F}(t+x) \leq \bar{F}(t) \bar{F}(x) \Longleftrightarrow \frac{\bar{F}(t+x)}{\bar{F}(t)} \leq \bar{F}(x) \Longleftrightarrow \frac{e^{-R(t+x)}}{e^{-R(t)}} \leq e^{-R(x)}
$$

onde $\mathrm{R}$ é a função de risco do tempo de vida $\mathrm{T}$, com

$$
R(t)=\int_{0}^{t} r(s) d s=-\log \bar{F}(t) .
$$

Observe que, para t e $\mathrm{x}$ reais positivos

$$
R(t+x)-R(t) \geq(t+x) \cdot \frac{R(x)}{x}=\left(1+\frac{x}{t}\right) \cdot R(x) \geq R(x) .
$$

A primeira desigualdade é válida pois T é IFRA, ou seja, R é star-shaped. Portanto

$$
\frac{e^{-R(t+x)}}{e^{-R(t)}} \leq e^{-R(x)}
$$

Concluímos que $\bar{F}(t+x) \leq \bar{F}(t) \bar{F}(x)$ para t e $\mathrm{x}$ reais positivos, e T é NBU.

(iii) Provamos a partir da classe NBU

Como T é NBU, isto é, $\bar{F}(t+x) \leq \bar{F}(t) \cdot \bar{F}(x)$, temos

$$
\begin{gathered}
\int_{0}^{\infty} \bar{F}(t+x) d x \leq \int_{0}^{\infty} \bar{F}(t) \cdot \bar{F}(x) d x \Rightarrow \int_{t}^{\infty} \bar{F}(s) d s \leq \bar{F}(t) \cdot \int_{0}^{\infty} \bar{F}(x) d x \\
\Rightarrow \int_{t}^{\infty} \bar{F}(s) d s \leq \bar{F}(t) \cdot E(T) .
\end{gathered}
$$

A primeira implicação resulta da mudança de variáveis $s=t+x$. Concluímos que T é NBUE.

Na próxima seção verificaremos as operações algébricas dos exemplos deste trabalho.

\section{A.4 Verificações para os Exemplos}

Exemplo A.4.1. Verificação do Exemplo 2.2.1.

\begin{tabular}{|c|c|c|c|c|c|c|}
\hline $\mathrm{k}$ & $f_{1 k}$ & $f_{2 k}$ & $\bar{F}_{1 k}$ & $\bar{F}_{2 k}$ & $\bar{F}_{2 k}$ & $\frac{f_{2 k}}{f_{1 k}}$ \\
\hline 0 & 0,5 & 0,4 & 1 & 1 & 1 & 0,8 \\
\hline 1 & 0,2 & 0,2 & 0,5 & 0,6 & 1,2 & 1,0 \\
\hline 2 & 0,3 & 0,4 & 0,3 & 0,4 & 1,3 & 1,3 \\
\hline
\end{tabular}

Com base na tabela acima, concluímos que $\mathbf{f}_{\mathbf{1}} \leq_{S T} \mathbf{f}_{\mathbf{2}}, \mathbf{f}_{\mathbf{1}} \leq_{H R} \mathbf{f}_{\mathbf{2}}$ e $\mathbf{f}_{\mathbf{1}} \leq_{L R} \mathbf{f}_{\mathbf{2}}$.

Mostraremos que as mesmas desigualdades valem para o D-espectro condicional:

$$
P\left(T_{1}>t\right)=e^{-2 t}\left(1+t+0,6 t^{2}\right)
$$




$$
P\left(T_{2}>t\right)=e^{-2 t}\left(1+1,2 . t+0,8 t^{2}\right) .
$$

Observe que

$$
\begin{gathered}
P\left(R_{1}>t\right)=e^{-2 t} \\
P\left(R_{2}>t\right)=e^{-2 t}(1+2 t) \\
P\left(R_{3}>t\right)=e^{-2 t}\left(1+2 t+\frac{4 t^{2}}{2}\right)=e^{-2 t}\left(1+2 t+2 t^{2}\right) .
\end{gathered}
$$

O k-ésimo componente do D-espectro condicional do i-ésimo network, $\mathrm{k}=1,2, \ldots \mathrm{n}$ e $\mathrm{i}=1,2$, dado $\left\{T_{i}>t\right\}$ é

$$
p_{i k}(t)=\frac{f_{i k} \cdot P\left(R_{k}>t\right)}{P\left(T_{i}>t\right)} .
$$

Temos:

$$
\begin{gathered}
p_{11}(t)=\frac{0,5 \cdot e^{-2 t}}{\left(1+t+0,6 t^{2}\right) e^{-2 t}}=\frac{0,5}{\left(1+t+0,6 t^{2}\right)} ; \\
p_{12}(t)=\frac{0,2 \cdot e^{-2 t}(1+2 t)}{\left(1+t+0,6 t^{2}\right) e^{-2 t}}=\frac{0,2+0,4 t}{\left(1+t+0,6 t^{2}\right)} ; \\
p_{13}(t)=\frac{0,3 \cdot e^{-2 t}\left(1+2 t+2 t^{2}\right)}{\left(1+t+0,6 t^{2}\right) e^{-2 t}}=\frac{\left.0,3+0,6 \cdot t+0,6 . t^{2}\right)}{\left(1+t+0,6 t^{2}\right)} ; \\
p_{21}(t)=\frac{0,4 \cdot e^{-2 t}}{\left(1+1,2 t+0,8 \cdot t^{2}\right) e^{-2 t}}=\frac{0,4}{\left(1+1,2 \cdot t+0,8 . t^{2}\right)} ; \\
p_{22}(t)=\frac{0,2 \cdot e^{-2 t}(1+2 t)}{\left(1+1,2 t+0,8 \cdot t^{2}\right) e^{-2 t}}=\frac{0,2+0,4 \cdot t}{\left(1+1,2 \cdot t+0,8 . t^{2}\right)} ; \\
p_{23}(t)=\frac{0,4 \cdot e^{-2 t}\left(1+2 t+2 \cdot t^{2}\right)}{\left(1+1,2 t+0,8 . t^{2}\right) e^{-2 t}}=\frac{0,4+0,8 \cdot t+0,8 . t^{2}}{\left(1+1,2 . t+0,8 . t^{2}\right)} .
\end{gathered}
$$

Portanto

$$
\begin{aligned}
& \sum_{j=2}^{3} p_{1 j}(t)=\frac{0,2+0,4 t}{\left(1+t+0,6 t^{2}\right)}+\frac{\left.0,3+0,6 \cdot t+0,6 \cdot t^{2}\right)}{\left(1+t+0,6 t^{2}\right)}=\frac{0,5+t+0,6 . t^{2}}{\left(1+t+0,6 t^{2}\right)} \\
& \sum_{j=3}^{3} p_{1 j}(t)=p_{13}(t)=\frac{\left.0,3+0,6 \cdot t+0,6 . t^{2}\right)}{\left(1+t+0,6 t^{2}\right)} \\
& \sum_{j=2}^{3} p_{2 j}(t)=\frac{0,2+0,4 . t}{\left(1+1,2 . t+0,8 . t^{2}\right)}+\frac{0,4+0,8 . t+0,8 . t^{2}}{\left(1+1,2 . t+0,8 . t^{2}\right)}=\frac{0,6+1,2 . t+0,8 . t^{2}}{\left(1+1,2 . t+0,8 . t^{2}\right)} \\
& \sum_{j=3}^{3} p_{2 j}(t)=p_{23}(t)=\frac{0,4+0,8 . t+0,8 . t^{2}}{\left(1+1,2 . t+0,8 . t^{2}\right)} .
\end{aligned}
$$

Verificamos a ordenação estocástica para o D-espectro condicional, ou seja, mostraremos que

$$
\sum_{j=k}^{3} p_{2 j}(t)-\sum_{j=k}^{3} p_{1 j}(t) \geq 0 .
$$


Temos:

$$
\begin{gathered}
\sum_{j=2}^{3} p_{2 j}(t)-\sum_{j=2}^{3} p_{1 j}(t)=\frac{0,6+1,2 \cdot t+0,8 . t^{2}}{\left(1+1,2 \cdot t+0,8 . t^{2}\right)}-\frac{0,5+t+0,6 . t^{2}}{\left(1+t+0,6 t^{2}\right)} \\
=\frac{\left(0,6+1,2 . t+0,8 \cdot t^{2}\right)\left(1+t+0,6 t^{2}\right)-\left(0,5+t+0,6 \cdot t^{2}\right)\left(1+1,2 . t+0,8 . t^{2}\right)}{\left(1+t+0,6 t^{2}\right) \cdot\left(1+1,2 \cdot t+0,8 \cdot t^{2}\right)} \\
=\frac{0,1+0,2 \cdot t+0,16 \cdot t^{2}}{\left(1+t+0,6 t^{2}\right) \cdot\left(1+1,2 \cdot t+0,8 \cdot t^{2}\right)} \geq 0,
\end{gathered}
$$

e

$$
\begin{gathered}
\sum_{j=3}^{3} p_{2 j}(t)-\sum_{j=3}^{3} p_{1 j}(t)=\frac{0,4+0,8 \cdot t+0,8 \cdot t^{2}}{\left(1+1,2 \cdot t+0,8 \cdot t^{2}\right)}-\frac{\left.0,3+0,6 \cdot t+0,6 \cdot t^{2}\right)}{\left(1+t+0,6 t^{2}\right)} \\
=\frac{0,4\left(1+2 t+2 t^{2}\right)}{1+1,2 \cdot t+0,8 \cdot t^{2}}-\frac{0,3\left(1+2 t+2 t^{2}\right)}{1+t+0,6 t^{2}} \\
=\frac{1+2 t+2 t^{2}}{\left(1+1,2 \cdot t+0,8 \cdot t^{2}\right)\left(1+t+0,6 t^{2}\right)} \cdot\left[0,4\left(1+t+0,6 t^{2}\right)-0,3\left(1+1,2 \cdot t+0,8 \cdot t^{2}\right)\right] \\
=\frac{1+2 t+2 t^{2}}{\left(1+1,2 \cdot t+0,8 \cdot t^{2}\right)\left(1+t+0,6 t^{2}\right)} \cdot\left[0,4+0,4 t+0,24 t^{2}-\left(0,3+0,36 \cdot t+0,24 \cdot t^{2}\right)\right] \\
=\frac{(0,1+0,04 \cdot t) \cdot\left(1+2 t+2 t^{2}\right)}{\left(1+t+0,6 t^{2}\right) \cdot\left(1+1,2 \cdot t+0,8 \cdot t^{2}\right)} \geq 0 .
\end{gathered}
$$

Concluímos que $\mathbf{p}_{\mathbf{1}}(\mathbf{t}) \leq_{S T} \mathbf{p}_{\mathbf{2}}(\mathbf{t})$.

Verificaremos a preservação da desigualdade através das taxas de falhas. Temos:

$$
\begin{aligned}
& \frac{\sum_{j=1}^{3} p_{2 j}(t)}{\sum_{j=1}^{3} p_{1 j}(t)}=1 \\
& \frac{\sum_{j=2}^{3} p_{2 j}(t)}{\sum_{j=2}^{3} p_{1 j}(t)}=\frac{\frac{0,6+1,2 . t+0,8 . t^{2}}{\left(1+1,2 . t+0,8 . t^{2}\right)}}{\frac{0,5+t+0,6 . t^{2}}{\left(1+t+0,6 t^{2}\right)}}=\frac{0,6+0,6 t+0,36 t^{2}+1,2 t+1,2 t^{2}+0,72 t^{3}+0,8 t^{2}+0,8 t^{3}+0,48 t^{4}}{0,5+t+0,6 t^{2}+0,6 t+1,2 t^{2}+0,72 t^{3}+0,4 t^{2}+0,8 t^{3}+0,48 t^{4}} \\
& =\frac{0,6+1,8 t+2,32 t^{2}+1,52 t^{3}+0,48 t^{4}}{0,5+1,6 t+2,2 t^{2}+1,52 t^{3}+0,48 t^{4}}=\frac{0,1+0,5+0,2 t+1,6 t+0,12 t^{2}+2,2 t^{2}+1,52 t^{3}+0,48 t^{4}}{0,5+1,6 t+2,2 t^{2}+1,52 t^{3}+0,48 t^{4}} \\
& =1+\frac{0,1+0,2 . t+0,12 \cdot t^{2}}{0,5+1,6 \cdot t+2,2 \cdot t^{2}+1,52 \cdot t^{3}+0,48 \cdot t^{4}} \geq 1=\frac{\sum_{j=1}^{3} p_{2 j}(t)}{\sum_{j=1}^{3} p_{1 j}(t)} \\
& \frac{\sum_{j=3}^{3} p_{2 j}(t)}{\sum_{j=3}^{3} p_{1 j}(t)}=\frac{\frac{0,4+0,8 \cdot t+0,8 \cdot t^{2}}{\left(1+1,2 \cdot t+0,8 . t^{2}\right)}}{\frac{\left.0,3+0,6 \cdot t+0,6 \cdot t^{2}\right)}{\left(1+t+0,6 t^{2}\right)}}=\frac{0,4}{0,3} \cdot \frac{\frac{1+2 t+2 t^{2}}{1+1,2 t+0,8 t^{2}}}{\frac{1+2 t+2 t^{2}}{1+t+0,6 t^{2}}} \\
& =\frac{4}{3} \cdot \frac{1+t+0,6 t^{2}}{1+1,2 t+0,8 t^{2}}=\frac{4+4 t+2,4 t^{2}}{3+3,6 t+2,4 t^{2}}=\frac{3+1+3,6 t+0,4 t+2,4 t^{2}}{3+3,6 t+2,4 t^{2}}
\end{aligned}
$$




$$
=\frac{3+3,6 t+2,4 t^{2}}{3+3,6 t+2,4 t^{2}}+\frac{1+0,4 t}{3+3,6 t+2,4 t^{2}}=1+\frac{1+0,4 \cdot t}{3+3,6 \cdot t+2,4 \cdot t^{2}} .
$$

Observe que

$$
\begin{gathered}
\frac{\sum_{j=3}^{3} p_{2 j}(t)}{\sum_{j=3}^{3} p_{1 j}(t)}-\frac{\sum_{j=2}^{3} p_{2 j}(t)}{\sum_{j=2}^{3} p_{1 j}(t)}=1+\frac{1+0,4 \cdot t}{3+3,6 \cdot t+2,4 \cdot t^{2}}-\left(1+\frac{0,1+0,2 \cdot t+0,12 \cdot t^{2}}{0,5+1,6 \cdot t+2,2 \cdot t^{2}+1,52 \cdot t^{3}+0,48 \cdot t^{4}}\right) \\
=\frac{(1+0,4 \cdot t) \cdot\left(0,5+1,6 \cdot t+2,2 \cdot t^{2}+1,52 \cdot t^{3}+0,48 \cdot t^{4}\right)}{\left(3+3,6 \cdot t+2,4 \cdot t^{2}\right)\left(0,5+1,6 \cdot t+2,2 \cdot t^{2}+1,52 \cdot t^{3}+0,48 \cdot t^{4}\right)} \\
-\frac{\left(0,1+0,2 \cdot t+0,12 \cdot t^{2}\right) \cdot\left(3+3,6 \cdot t+2,4 \cdot t^{2}\right)}{\left(3+3,6 \cdot t+2,4 \cdot t^{2}\right)\left(0,5+1,6 \cdot t+2,2 \cdot t^{2}+1,52 \cdot t^{3}+0,48 \cdot t^{4}\right)} \\
=\frac{0,5+1,6 t+2,2 t^{2}+1,52 t^{3}+0,48 t^{4}+0,2 t+0,64 t^{2}+0,88 t^{3}+0,608 t^{4}+0,192 t^{5}}{\left(3+3,6 \cdot t+2,4 \cdot t^{2}\right)\left(0,5+1,6 \cdot t+2,2 \cdot t^{2}+1,52 \cdot t^{3}+0,48 \cdot t^{4}\right)} \\
-\frac{\left(0,3+0,36 t+0,24 t^{2}+0,6 t+0,72 t^{2}+0,48 t^{3}+0,36 t^{2}+0,432 t^{3}+0,288 t^{4}\right)}{\left(3+3,6 \cdot t+2,4 \cdot t^{2}\right)\left(0,5+1,6 \cdot t+2,2 \cdot t^{2}+1,52 \cdot t^{3}+0,48 \cdot t^{4}\right)} \\
=\frac{0,2+0,84 \cdot t+1,52 \cdot t^{2}+1,488 \cdot t^{3}+0,8 \cdot t^{4}+0,192 t^{5}}{\left(3+3,6 \cdot t+2,4 \cdot t^{2}\right)\left(0,5+1,6 \cdot t+2,2 \cdot t^{2}+1,52 \cdot t^{3}+0,48 \cdot t^{4}\right)} \geq 0 .
\end{gathered}
$$

Concluímos que $\frac{\sum_{j=1}^{3} p_{2 j}(t)}{\sum_{j=1}^{3} p_{1 j}(t)} \leq \frac{\sum_{j=2}^{3} p_{2 j}(t)}{\sum_{j=2}^{3} p_{1 j}(t)} \leq \frac{\sum_{j=3}^{3} p_{2 j}(t)}{\sum_{j=3}^{3} p_{1 j}(t)}$ e $\mathbf{p}_{\mathbf{1}}(\mathbf{t}) \leq_{H R} \mathbf{p}_{\mathbf{2}}(\mathbf{t})$.

Avaliaremos a desigualdade através da razão de verossimilhanças. Temos:

$$
\begin{gathered}
\frac{p_{21}(t)}{p_{11}(t)}=\frac{\frac{0,4}{1+1,2 . t+0,8 . t^{2}}}{\frac{0,5}{1+t+0,6 \cdot t^{2}}}=\frac{0,8 \cdot\left(1+t+0,6 \cdot t^{2}\right)}{1+1,2 \cdot t+0,8 \cdot t^{2}} \\
\frac{p_{22}(t)}{p_{12}(t)}=\frac{\frac{0,2(1+2 t)}{1+1,2 . t+0,8 \cdot t^{2}}}{\frac{0,2(1+2 t)}{1+t+0,6 \cdot t^{2}}}=\frac{1+t+0,6 \cdot t^{2}}{1+1,2 \cdot t+0,8 . t^{2}} ; \\
\frac{p_{23}(t)}{p_{13}(t)}=\frac{\frac{0,4\left(1+2 t+2 t^{2}\right)}{1+1,2 . t+0,8 . t^{2}}}{\frac{0,3\left(1+2 t+2 t^{2}\right)}{1+t+0,6 \cdot t^{2}}}=\frac{4 \cdot\left(1+t+0,6 \cdot t^{2}\right)}{3 \cdot\left(1+1,2 \cdot t+0,8 \cdot t^{2}\right)} .
\end{gathered}
$$

Concluímos que $\frac{p_{21}(t)}{p_{11}(t)} \leq \frac{p_{22}(t)}{p_{12}(t)} \leq \frac{p_{23}(t)}{p_{13}(t)}$ e $\mathbf{p}_{\mathbf{1}}(\mathbf{t}) \leq_{L R} \mathbf{p}_{\mathbf{2}}(\mathbf{t})$.

Exemplo A.4.2 Verificação para o Exemplo 2.3.1. Sabemos, pelo Exemplo 2.2.1, que $\mathbf{f}_{\mathbf{1}} \leq_{S T} \mathbf{f}_{\mathbf{2}}$, $\mathbf{f}_{1} \leq_{H R} \mathbf{f}_{\mathbf{2}}$ e $\mathbf{f}_{1} \leq_{L R} \mathbf{f}_{\mathbf{2}}$.

Pretende-se estudar a preservação das desigualdades estocásticas para os networks.

Para analisar a preservação da ordenação estocástica, temos:

$$
\begin{gathered}
P\left(T_{1}>t\right)=1 . e^{-2 t}+0,5 . e^{-2 t}(2 . t)+0,3 . e^{-2 t} \frac{(2 . t)^{2}}{2}=e^{-2 t}\left(1+t+0,6 . t^{2}\right) \\
P\left(T_{2}>t\right)=1 . e^{-2 t}+0,6 . e^{-2 t}(2 . t)+0,4 . e^{-2 t} \frac{(2 . t)^{2}}{2}=e^{-2 t}\left(1+1,2 . t+0,8 . t^{2}\right) .
\end{gathered}
$$


Concluímos que $P\left(T_{1}>t\right) \leq P\left(T_{2}>t\right)$ e $T_{1} \leq_{S T} T_{2}$.

Para estudar a preservação da desigualdade através de taxas de falhas, temos

$$
\begin{gathered}
\frac{P\left(T_{2}>t\right)}{P\left(T_{1}>t\right)}=\frac{1+1,2 . t+0,8 . t^{2}}{1+t+0,6 . t^{2}} ; \\
\frac{d}{d t} \frac{P\left(T_{2}>t\right)}{P\left(T_{1}>t\right)}=\frac{0,2+0,4 . t+0,08 . t^{2}}{\left(1+t+0,6 . t^{2}\right)^{2}} \geq 0 .
\end{gathered}
$$

Concluímos que $\frac{P\left(T_{2}>t\right)}{P\left(T_{1}>t\right)}$ é crescente em $\mathrm{t}>0$ e, portanto, $T_{1} \leq_{H R} T_{2}$.

Analisaremos a preservação da desigualdade através da razão de verossimilhanças.

A função densidade de probabilidade do tempo de vida do i-ésimo network no instante $\mathrm{t}>0$, $\mathrm{i}=1,2$ é $h_{i}(t)=\sum_{k=1}^{3} f_{i k} \cdot g_{k}(t)$.

Seja $g_{k}(t)$ é a função densidade de probabilidade de $R_{k}, \mathrm{k}=1,2 \ldots . \mathrm{n}$.

Então

$$
\begin{gathered}
h_{1}(t)=0,5.2 . e^{-2 t}+0,2.2 . e^{-2 t}(2 . t)+0,3.2 . e^{-2 t} \cdot \frac{(2 t)^{2}}{2}=e^{-2 t} \cdot\left(1+0,8 . t+1,2 . t^{2}\right) ; \\
h_{2}(t)=0,4.2 . e^{-2 t}+0,2.2 . e^{-2 t}(2 . t)+0,4.2 . e^{-2 t} \cdot \frac{(2 t)^{2}}{2}=e^{-2 t} \cdot\left(0,8+0,8 . t+1,6 . t^{2}\right) ; \\
\frac{h_{2}(t)}{h_{1}(t)}=\frac{0,8+0,8 . t+1,6 . t^{2}}{1+0,8 . t+1,2 . t^{2}} .
\end{gathered}
$$

Portanto

$$
\begin{gathered}
\frac{d}{d t} \frac{h_{2}(t)}{h_{1}(t)}=\frac{(0,8+3,2 t)\left(1+0,8 t+1,2 t^{2}\right)-\left(0,8+0,8 t+1,6 t^{2}\right)(0,8+2,4 t)}{\left(1+0,8 t+1,2 t^{2}\right)^{2}} \\
=\frac{0,8+0,64 t+0,96+3,2 t+2,56 t^{2}+3,84 t^{3}-0,64-0,64 t-1,28 t^{2}-1,92 t-1,92 t^{2}-3,84 t^{3}}{\left(1+0,8 t+1,2 t^{2}\right)^{2}} \\
=\frac{0,16+1,28 . t+0,32 \cdot t^{2}}{\left(1+0,8 . t+1,2 \cdot t^{2}\right)^{2}} \geq 0 .
\end{gathered}
$$

Concluímos que $\frac{h_{2}(t)}{h_{1}(t)}$ é crescente em $\mathrm{t}>0$ e, portanto, $T_{1} \leq_{L R} T_{2}$.

Exemplo A.4.3. Verificação do Exemplo 2.4.1.

A confiabilidade do i-ésimo network é

$$
P\left(T_{i}>t\right)=\sum_{k=0}^{2} \bar{F}_{k} \cdot P(N(t)=k),
$$

onde $\bar{F}_{i k}$ é a função de sobrevivência do D-espectro do i-ésimo network a k falhas de seus links, $\mathrm{i}=1,2, \mathrm{k}=0,1,2 \ldots \mathrm{n}-1$.

Temos: $\bar{F}_{11}=\bar{F}_{21}=1, \bar{F}_{12}=0,6, \bar{F}_{22}=0,8, \bar{F}_{13}=0,2, \bar{F}_{23}=0,4$. 
O k-ésimo componente do D-espectro condicional do i-ésimo network no instante t é

$$
p_{i k}(t)=\frac{f_{i k} \cdot P\left(R_{k}>t\right)}{P\left(T_{i}>t\right)},
$$

onde $R_{k}$ é o instante da k-ésima falha dos links.

Assim

$$
\begin{gathered}
P\left(R_{1}>t\right)=e^{-t} ; \\
P\left(R_{2}>t\right)=e^{-t}(1+t) ; \\
P\left(R_{3}>t\right)=e^{-t}\left(1+t+\frac{t^{2}}{2}\right) ; \\
P\left(T_{1}>t\right)=e^{-t}\left(1+0,6 t+0,1 t^{2}\right) ; \\
P\left(T_{2}>t\right)=e^{-t}\left(1+0,8 t+0,2 t^{2}\right) .
\end{gathered}
$$

As funções densidades de probabilidade dos tempos de vidas dos networks são

$$
\begin{gathered}
h_{1}(t)=\frac{-d P\left(T_{1}>t\right)}{d t}=e^{-t}\left(1+0,6 t+0,1 t^{2}\right)-e^{-t}(0,6+0,2 t) \\
=e^{-t}\left(0,4+0,4 t+0,1 t^{2}\right)=0,2 \cdot e^{-t} \cdot(2+t)^{2} ; \\
h_{2}(t)=\frac{-d P\left(T_{2}>t\right)}{d t}=e^{-t}\left(1+0,8 t+0,2 t^{2}\right)-e^{-t}(0,8+0,4 t) \\
=e^{-t}\left(0,2+0,4 t+0,2 t^{2}\right)=0,2 \cdot e^{-t} \cdot\left(1+2 t+t^{2}\right)=0,2 \cdot e^{-t} \cdot(1+t)^{2} .
\end{gathered}
$$

Verificaremos que $\mathbf{p}_{\mathbf{1}}(\mathbf{t}) \leq_{L R} \mathbf{p}_{\mathbf{2}}(\mathbf{t})$, isto é $\frac{p_{2 k}(t)}{p_{1 k}(t)}$ é crescente em k.

Temos:

$$
\begin{gathered}
p_{11}(t)=\frac{f_{11} \cdot P\left(R_{1}>t\right)}{P\left(T_{1}>t\right)}=\frac{0,4 \cdot e^{-t}}{e^{-t}\left(1+0,6 t+0,4 t^{2}\right)}=\frac{0,4}{1+0,6 t+0,1 t^{2}} ; \\
p_{12}(t)=\frac{f_{12} \cdot P\left(R_{2}>t\right)}{P\left(T_{1}>t\right)}=\frac{\left(0,4(1+t) \cdot e^{-t}\right.}{e^{-t}\left(1+0,6 t+0,4 t^{2}\right)}=\frac{(0,4+0,4 t)}{\left(1+0,6 t+0,1 t^{2}\right)} \\
p_{13}(t)=\frac{f_{13} \cdot P\left(R_{3}>t\right)}{P\left(T_{1}>t\right)}=\frac{0,2\left(1+t+\frac{t^{2}}{2}\right) \cdot e^{-t}}{e^{-t}\left(1+0,6 t+0,4 t^{2}\right)}=\frac{0,2+0,2 t+0,1 \cdot t^{2}}{1+0,6 t+0,1 t^{2}} ; \\
p_{21}(t)=\frac{f_{21} \cdot P\left(R_{2}>t\right)}{P\left(T_{2}>t\right)}=\frac{0,2 \cdot e^{-t}}{e^{-t}\left(1+0,8 t+0,2 t^{2}\right)}=\frac{0,2}{1+0,8 t+0,2 t^{2}} ; \\
p_{22}(t)=\frac{f_{22} \cdot P\left(R_{2}>t\right)}{P\left(T_{2}>t\right)}=\frac{\left(0,4(1+t) \cdot e^{-t}\right.}{e^{-t}\left(1+0,8 t+0,2 t^{2}\right)}=\frac{(0,4+0,4 t)}{\left(1+0,8 t+0,2 t^{2}\right)} ; \\
p_{23}(t)=\frac{f_{23} \cdot P\left(R_{3}>t\right)}{P\left(T_{2}>t\right)}=\frac{0,4\left(1+t+\frac{t^{2}}{2}\right) \cdot e^{-t}}{e^{-t}\left(1+0,8 t+0,2 t^{2}\right)}=\frac{0,4+0,4 t+0,2 \cdot t^{2}}{1+0,8 t+0,2 t^{2}} .
\end{gathered}
$$

Portanto

$$
\frac{p_{21}(t)}{p_{11}(t)}=\frac{\frac{0,2}{1+0,8 t+0,2 t^{2}}}{\frac{0,4}{1+0,6 t+0,1 t^{2}}}=\frac{1}{2} \cdot\left(\frac{1+0,6 t+0,1 t^{2}}{1+0,8 t+0,2 t^{2}}\right) ;
$$




$$
\begin{aligned}
& \frac{p_{22}(t)}{p_{12}(t)}=\frac{\frac{0,4(1+t)}{1+0,8 t+0,2 t^{2}}}{\frac{0,4(1+t)}{1+0,6 t+0,1 t^{2}}}=1 \cdot\left(\frac{1+0,6 t+0,1 t^{2}}{1+0,8 t+0,2 t^{2}}\right) ; \\
& \frac{p_{23}(t)}{p_{13}(t)}=\frac{\frac{0,4\left(1+t+\frac{t^{2}}{2}\right)}{1+0,8 t+0,2 t^{2}}}{\frac{0,2\left(1+t+\frac{t^{2}}{2}\right)}{1+0,6 t+0,1 t^{2}}}=2 \cdot\left(\frac{1+0,6 t+0,1 t^{2}}{1+0,8 t+0,2 t^{2}}\right) .
\end{aligned}
$$

Concluímos que $\frac{p_{21}(t)}{p_{11}(t)} \leq \frac{p_{22}(t)}{p_{12}(t)} \leq \frac{p_{23}(t)}{p_{13}(t)}$ e $\mathbf{p}_{\mathbf{1}}(\mathbf{t}) \leq_{L R} \mathbf{p}_{\mathbf{2}}(\mathbf{t})$

Pelo Teorema A.2.1 isto implica $\mathbf{p}_{\mathbf{1}}(\mathbf{t}) \leq_{H R} \mathbf{p}_{\mathbf{2}}(\mathbf{t})$ e consequentemente $\mathbf{p}_{\mathbf{1}}(\mathbf{t}) \leq_{S T} \mathbf{p}_{\mathbf{2}}(\mathbf{t})$.

A função densidade do tempo de vida residual do i-ésimo network em $\mathrm{x}$, dado $\left\{T_{i}>t\right\}$ é

$$
h_{i}(x \mid t)=\frac{h_{i}(x+t)}{P\left(T_{i}>t\right)}
$$

Verificaremos que $\left\{T_{1}-t \mid T_{1}>t\right\} \leq_{L R}\left\{T_{2}-t \mid T_{2}>t\right\}$, isto é $\frac{h_{2}(x \mid t)}{h_{1}(x \mid t)}$ é crescente em $\mathrm{x}$.

Temos:

$$
\frac{h_{2}(x \mid t)}{h_{1}(x \mid t)}=\frac{\frac{h_{2}(x+t)}{P\left(T_{2}>t\right)}}{\frac{h_{1}(x+t)}{P\left(T_{1}>t\right)}}=\frac{P\left(T_{1}>t\right)}{P\left(T_{2}>t\right)} \frac{h_{2}(t+x)}{h_{1}(t+x)} .
$$

Como $\frac{P\left(T_{1}>t\right)}{P\left(T_{2}>t\right)}$ não depende de $\mathrm{x}$ e é positivo, basta verificar que $\frac{h_{2}(t+x)}{h_{1}(t+x)}$ é crescente em $\mathrm{x}$. Temos:

$$
\begin{gathered}
\frac{h_{2}(t+x)}{h_{1}(t+x)}=\frac{(1+t+x)^{2}}{(2+t+x)^{2}}=\left(\frac{(1+t+x)}{(2+t+x)}\right)^{2} \\
\frac{d}{d x} \frac{h_{2}(t+x)}{h_{1}(t+x)}=2 \cdot \frac{1+t+x}{2+t+x} \cdot\left(\frac{(2+t+x)-(1+t+x)}{(2+t+x)^{2}}\right)=\frac{2(1+t+x)}{(2+t+x)^{3}} \geq 0 .
\end{gathered}
$$

Concluímos que $\frac{h_{2}(x \mid t)}{h_{1}(x \mid t)}$ é crescente em x e $\left\{T_{1}-t \mid T_{1}>t\right\} \leq_{L R}\left\{T_{2}-t \mid T_{2}>t\right\}$. Pelo Teorema A.2.1, isto implica $\left\{T_{1}-t \mid T_{1}>t\right\} \leq_{H R}\left\{T_{2}-t \mid T_{2}>t\right\}$ e consequentemente $\left\{T_{1}-t \mid T_{1}>t\right\} \leq_{S T}$ $\left\{T_{2}-t \mid T_{2}>t\right\}$.

Exemplo A.4.4. Verificação do Exemplo 3.1.1.

Considere $\bar{F}$ um vetor com a função de sobrevivência do D-espectro, da forma $\bar{F}=\left(\bar{F}_{0}, \bar{F}_{1}, \bar{F}_{2}\right)=(1 ; 0,5 ; 0,3)$.

Verificamos que

$$
\frac{\bar{F}_{2}}{\bar{F}_{1}} \leq \frac{\bar{F}_{1}}{\bar{F}_{0}} \Longleftrightarrow \bar{F}_{1}^{2}-\bar{F}_{2} \cdot \bar{F}_{0} \leq 0
$$

Como $\bar{F}_{1}^{2}-\bar{F}_{0} \cdot \bar{F}_{2}=0,5^{2}-1.0,3=-0,05 \leq 0$, concluímos que f é DFR.

Exemplo A.4.5. Verificação do Exemplo 3.1.2.

Verificamos a classe de distribuições do D-espectro f. Portanto

$$
\frac{\bar{F}_{1}}{\bar{F}_{0}}-\frac{\bar{F}_{2}}{\bar{F}_{1}}=\frac{0,4}{1}-\frac{0,3}{0,4}=0,4-0,75=-0,35 \leq 0
$$

onde $\bar{F}_{k}$ é a função de sobrevivência do D-espectro após k falhas de seus links.

Então f é DFR. 
Verificamos a convexidade da função de risco $\Lambda(t)$.

Portanto $\frac{d \Lambda(t)}{d t}=e^{-t}$ e $\frac{d^{2} \Lambda(t)}{d t^{2}}=-e^{-t}<0$.

Então a função de risco é côncava.

A confiabilidade do network é

$$
\begin{gathered}
\bar{H}(t)=\sum_{k=0}^{2} \bar{F}_{k} \cdot P(N(t)=k) \\
=e^{-\left(1-e^{-t}\right)}\left(1+0,4 .\left(1-e^{-t}\right)+0,15\left(1-e^{-t}\right)^{2}\right)=e^{-1} e^{-e^{-t}} \cdot\left(1,55-0,7 \cdot e^{-t}+0,15 e^{-2 t}\right) .
\end{gathered}
$$

A função densidade de probabilidade de $\mathrm{T}$ é

$$
\begin{gathered}
h(t)=-\frac{d \bar{H}(t)}{d t}=-\left[e^{-\left(1-e^{-t}\right)} \cdot\left(-e^{-t}\right)\left(1,55-0,7 \cdot e^{-t}+0,15 e^{-2 t}\right)+e^{-\left(1-e^{-t}\right)} \cdot\left(0,7 e^{-t}-0,3 e^{-2 t}\right)\right] \\
=e^{-\left(1-e^{-t}\right)}\left(0,85 e^{-t}-0,4 e^{-2 t}+0,15 e^{-3 t}\right) .
\end{gathered}
$$

A taxa de falha do network é

$$
r(t)=\frac{h(t)}{\bar{H}(t)}=\frac{e^{-\left(1-e^{-t}\right)}\left(0,85 e^{-t}-0,4 e^{-2 t}+0,15 e^{-3 t}\right)}{e^{-1} e^{-e^{-t}} \cdot\left(1,55-0,7 . e^{-t}+0,15 e^{-2 t}\right)}=\frac{0,85 e^{-t}-0,4 e^{-2 t}+0,15 e^{-3 t}}{1,55-0,7 \cdot e^{-t}+0,15 e^{-2 t}} .
$$

Verificamos que a taxa de falha é decrescente

$$
\begin{gathered}
\frac{d r(t)}{d t}=\frac{\left(-0,85 e^{-t}+0,8 e^{-2 t}-0,45 e^{-3 t}\right)\left(1,55-0,7 . e^{-t}+0,15 e^{-2 t}\right)}{\left(1,55-0,7 \cdot e^{-t}+0,15 e^{-2 t}\right)^{2}} \\
-\frac{\left(0,85 e^{-t}-0,4 e^{-2 t}+0,15 e^{-3 t}\right)\left(0,7 . e^{-t}-0,3 e^{-2 t}\right)}{\left(1,55-0,7 \cdot e^{-t}+0,15 e^{-2 t}\right)^{2}} \\
=\frac{-1,3175 e^{-t}+0,595 e^{-2 t}-0,1275 e^{-3 t}+1,24 e^{-2 t}-0,56 e^{-3 t}+0,12 e^{-4 t}-0,6975 e^{-3 t}+0,315 e^{-4 t}}{\left(1,55-0,7 \cdot e^{-t}+0,15 e^{-2 t}\right)^{2}} \\
-\frac{0,0675 e^{-5 t}+0,595 e^{-2 t}-0,255 e^{-3 t}-0,28 e^{-3 t}+0,12 e^{-4 t}+0,105 e^{-4 t}-0,045 e^{-5 t}}{\left(1,55-0,7 \cdot e^{-t}+0,15 e^{-2 t}\right)^{2}} \\
=-\frac{1,3175 e^{-t}+1,24 e^{-2 t}-0,85 e^{-3 t}+0,210 e^{-4 t}-0,0225 e^{-5 t}}{\left(1,55-0,7 . e^{-t}+0,15 e^{-2 t}\right)^{2}} \leq 0 .
\end{gathered}
$$

A desigualdade é válida pois:

- $1,3175 e^{-t} \geq 1,24 e^{-2 t}$;

- $0,85 e^{-3 t} \geq 0,210 e^{-4 t}$;

- $e^{-a t} \leq 1, \forall a, t \geq 0$

- $e^{-a t} \leq e^{-b t}, a \geq b \geq 0, \forall t \geq 0$.

Concluímos que $\bar{H}(t)$ é log-convexa e T é DFR.

Exemplo A.4.6. Verificação do Exemplo 3.1.3.

Sabemos, pelo Exemplo 3.1.1, que f é DFR. 
A confiabilidade do network no instante t $>0$ é

$$
\bar{H}(t)=P(T>t)=\sum_{k=0}^{2} \bar{F}_{k} \cdot P(N(t)=k)=e^{-e^{t}} \cdot\left(1+0,5 \cdot e^{t}+0,15 \cdot\left(e^{t}\right)^{2}\right) .
$$

Então

$$
\begin{aligned}
& \log \bar{H}(t)=-e^{t}+\log \left(1+0,5 \cdot e^{t}+0,15 \cdot\left(e^{t}\right)^{2}\right) ; \\
& \frac{d}{d t} \log \bar{H}(t)=-e^{t}+\frac{0,5 \cdot e^{t}+0,3 \cdot\left(e^{t}\right)^{2}}{1+0,5 \cdot e^{t}+0,15 \cdot\left(e^{t}\right)^{2}}=\frac{-e^{t}-0,5 e^{2 t}-0,15 e^{3 t}+0,5 e^{t}+0,3 e^{2 t}}{1+0,5 \cdot e^{t}+0,15 \cdot\left(e^{t}\right)^{2}} \\
& =\frac{-0,5 \cdot e^{t}-0,2 \cdot e^{2 t}-0,15 \cdot e^{3 t}}{1+0,5 \cdot e^{t}+0,15 \cdot\left(e^{2 t}\right)} \\
& \frac{d^{2}}{d t^{2}} \log \bar{H}(t)=\frac{\left(-0,5 . e^{t}-0,4 . e^{2 t}-0,45 . e^{3 t}\right)\left(1+0,5 \cdot e^{t}+0,15 \cdot e^{2 t}\right)}{\left(1+0,5 . e^{t}+0,15 \cdot e^{2 t}\right)^{2}} \\
& -\frac{\left(-0,5 . e^{t}-0,2 . e^{2 t}-0,15 . e^{3 t}\right)\left(0,5 . e^{t}+0,3 . e^{2 t}\right)}{\left(1+0,5 . e^{t}+0,15 . e^{2 t}\right)^{2}} \\
& =\frac{-0,5 e^{t}-0,25 e^{2 t}-0,075 e^{3 t}-0,4 e^{2 t}-0,2 e^{3 t}-0,06 e^{4 t}-0,45 e^{3 t}-0,225 e^{4 t}-0,0675 e^{5 t}}{\left(1+0,5 . e^{t}+0,15 . e^{2 t}\right)^{2}} \\
& +\frac{0,25 e^{2 t}+0,15 e^{3 t}+0,1 e^{3 t}+0,06 e^{4 t}+0,075 e^{4 t}+0,045 e^{5 t}}{\left(1+0,5 . e^{t}+0,15 . e^{2 t}\right)^{2}} \\
& =\frac{-0,5 . e^{t}-0,4 . e^{2 t}-0,475 . e^{3 t}-0,15 \cdot e^{4 t}-0,1125 . e^{5 t}}{\left(1+0,5 . e^{t}+0,15 \cdot e^{2 t}\right)^{2}} \leq 0 .
\end{aligned}
$$

Portanto, $\frac{d}{d t} \log \bar{H}(t)$ é decrescente em $\mathrm{t}>0$.

Observe que $\frac{d}{d t} \Lambda(t)=e^{t} \geq 0$ e, portanto, $\Lambda(t)$ é convexa.

Concluímos que $\bar{H}(t)$ é log-côncava e T é IFR, sentido contrário à classe de distribuição do D-espectro $\mathbf{f}$.

Exemplo A.4.7. Verificação do Exemplo 3.1.4.

Vamos avaliar a classe IFRA (DFRA) para o D-espectro.

Considere $\bar{F}^{*}$ um vetor tal que, para este exemplo

$\bar{F}^{*}=\left(\bar{F}_{1}^{\frac{1}{1}}, \bar{F}_{2}^{\frac{1}{2}}\right)=\left(0,5^{\frac{1}{1}}, 0,3^{\frac{1}{2}}\right)=(0,5 ; 0,55)$.

Como $\bar{F}_{k}^{\frac{1}{k}}$ é crescente em $\mathrm{k}=1,2$, concluímos que f é DFRA.

Exemplo A.4.8. Verificação do Exemplo 3.1.5.

Pelo Exemplo 3.1.4, sabemos que f é DFRA.

Verificamos se a função de risco é star-shaped

$$
\frac{d}{d t} \frac{\Lambda(t)}{t}=\frac{d}{d t} \frac{\sqrt{t}}{t}=\frac{d}{d t} t^{-\frac{1}{2}}=-\frac{1}{2} \cdot t^{-\frac{3}{2}} \leq 0 .
$$

Portanto, $\frac{\Lambda(t)}{t}$ é decrescente, e $\Lambda(t)$ é antistar-shaped.

A confiabilidade do network é

$$
\bar{H}(t)=\sum_{k=0}^{2} \bar{F}_{k} \cdot P(N(t)=k)=e^{-\sqrt{t}}\left(1+\sqrt{t}+\frac{t}{2}\right) .
$$


Portanto

$\frac{\log \bar{H}(t)}{t}=-\frac{\sqrt{t}}{t}+\frac{1}{t} \cdot \log \left(1+\sqrt{t}+\frac{t}{2}\right)=t^{\frac{1}{2}-1}+\frac{1}{t} \log \left(1+t^{\frac{1}{2}}+\frac{t}{2}\right)=t^{-\frac{1}{2}}+\frac{1}{t} \log \left(1+t^{\frac{1}{2}}+\frac{t}{2}\right)$.

Verificamos que $\frac{\log \bar{H}(t)}{t}$ é crescente em t.

$$
\frac{d}{d t} \frac{\log \bar{H}(t)}{t}=\frac{1}{2} \cdot t^{-\frac{3}{2}}+\frac{\frac{1}{2} \cdot t^{-\frac{1}{2}}+\frac{1}{2}}{1+t^{\frac{1}{2}}+\frac{t}{2}} \geq 0
$$

Portanto, $\log \bar{H}(t)$ é star-shaped e T é DFRA.

Exemplo A.4.9. Verificação do Exemplo 3.1.6.

Pelo Exemplo 3.1.4 sabemos que o D-espectro f é DFRA.

A confiabilidade do network no instante $t>0$ é

$$
\bar{H}(t)=e^{-t^{2}}\left(1+0,5 . t^{2}+0,15 . t^{4}\right)
$$

$\mathrm{e}$

$$
\begin{gathered}
\frac{1}{t} \log \bar{H}(t)=\frac{1}{t} \cdot\left[-t^{2}+\log \left(1+0,5 \cdot t^{2}+0,15 \cdot t^{4}\right)\right]=-t+\frac{\log \left(1+0,5 \cdot t^{2}+0,15 \cdot t^{4}\right)}{t} \\
\frac{d}{d t} \frac{\log \bar{H}(t)}{t}=-1+\frac{\frac{\left(t+0,6 \cdot t^{3}\right) \cdot t}{1+0,5 \cdot t^{2}+0,15 \cdot t^{4}}-\log \left(1+0,5 \cdot t^{2}+0,15 \cdot t^{4}\right)}{t^{2}} \\
=\frac{-t^{2}+\frac{t^{2}+0,6 t^{4}}{1+0,5 \cdot t^{2}+0,15 \cdot t^{4}}-\log \left(1+0,5 \cdot t^{2}+0,15 \cdot t^{4}\right)}{t^{2}} \\
=\frac{-t^{2}\left(1+0,5 \cdot t^{2}+0,15 \cdot t^{4}\right)+t^{2}+0,6 t^{4}-\left(1+0,5 \cdot t^{2}+0,15 \cdot t^{4}\right) \cdot \log \left(1+0,5 \cdot t^{2}+0,15 \cdot t^{4}\right)}{\left(1+0,5 \cdot t^{2}+0,15 \cdot t^{4}\right) \cdot t^{2}} \\
=\frac{-t^{2}-0,5 \cdot t^{4}-0,15 \cdot t^{6}+t^{2}+0,6 t^{4}-\left(1+0,5 \cdot t^{2}+0,15 \cdot t^{4}\right) \cdot \log \left(1+0,5 \cdot t^{2}+0,15 \cdot t^{4}\right)}{\left(1+0,5 \cdot t^{2}+0,15 \cdot t^{4}\right) \cdot t^{2}} \\
=\frac{0,1 \cdot t^{4}-0,15 \cdot t^{6}-\left(1+0,5 \cdot t^{2}+0,15 \cdot t^{4}\right) \cdot \log \left(1+0,5 \cdot t^{2}+0,15 \cdot t^{4}\right)}{\left(1+0,5 \cdot t^{2}+0,15 \cdot t^{4}\right) \cdot t^{2}} .
\end{gathered}
$$

Como o denominador é positivo, é suficiente analisar o sinal do numerador.

Seja

$$
\phi(t)=0,1 . t^{4}-0,15 \cdot t^{6}-\left(1+0,5 \cdot t^{2}+0,15 \cdot t^{4}\right) \cdot \log \left(1+0,5 \cdot t^{2}+0,15 \cdot t^{4}\right) .
$$

Temos: $\phi(0)=0$ e

$$
\begin{gathered}
\frac{d \phi(t)}{d t}=0,4 t^{3}-0,9 t^{5}-\left[\left(t+0,6 t^{3}\right) \cdot \log \left(1+0,5 \cdot t^{2}+0,15 \cdot t^{4}\right)+\left(1+0,5 \cdot t^{2}+0,15 \cdot t^{4}\right) \cdot \frac{\left(t+0,6 t^{3}\right.}{1+0,5 \cdot t^{2}+0,15 \cdot t^{4}}\right] \\
=0,4 t^{3}-0,9 t^{5}-\left[\left(t+0,6 t^{3}\right) \cdot \log \left(1+0,5 \cdot t^{2}+0,15 \cdot t^{4}\right)+\left(1+0,5 \cdot t^{2}+0,15 \cdot t^{4}\right) \cdot \frac{t+0,6 t^{3}}{1+0,5 \cdot t^{2}+0,15 \cdot t^{4}}\right] \\
=0,4 t^{3}-0,9 t^{5}-\left[\left(t+0,6 t^{3}\right) \cdot \log \left(1+0,5 \cdot t^{2}+0,15 \cdot t^{4}\right)+t+0,6 t^{3}\right] \\
=-t-0,2 t^{3}-0,9 t^{5}-\left(t+0,6 t^{3}\right) \cdot \log \left(1+0,5 \cdot t^{2}+0,15 \cdot t^{4}\right) \leq 0 .
\end{gathered}
$$


Então $\phi(t) \leq 0$ e portanto

$$
\frac{d}{d t} \frac{\log \bar{H}(t)}{t} \leq 0
$$

Concluímos que $\frac{1}{t} \log \bar{H}(t)$ é decrescente em t e T é IFRA, em sentido contrário ao D-espectro f. Observe que $\frac{\Lambda(t)}{t}=\frac{t^{2}}{t}=t$ é crescente e, portanto, a função de risco $\Lambda(t)=t^{2}$ 'e star-shaped. Tal condição não implica a preservação da classe do D-espectro para o tempo de vida.

Exemplo A.4.10. Verificação do Exemplo 3.1.7.

Como $\bar{F}_{2}-\bar{F}_{1} \cdot \bar{F}_{1}=0,3-0,5.0,5=0,05>0$, o D-espectro é NWU.

Exemplo A.4.11. Verificação do Exemplo 3.1.8.

Como $\bar{F}_{2}-\bar{F}_{1} \cdot \bar{F}_{1}=0,6-0,9.0,9=-0,21 \leq 0, \mathbf{f}$ é NBU.

Observe que $\Lambda(t+x)=\lambda . t+\lambda \cdot x=\Lambda(t)+\Lambda(x)$, ou seja, $\Lambda(t)=\lambda . t$ é superaditiva e subaditiva.

A confiabilidade do network é

$$
\bar{H}(t)=\sum_{k=0}^{2} \bar{F}_{k} \cdot P(N(t)=k)=e^{-t}\left(1+0,9 \cdot t+0,3 \cdot t^{2}\right) .
$$

Portanto

$$
\begin{gathered}
\bar{H}(t+x)-\bar{H}(t) \bar{H}(x)=e^{-(t+x)}\left(1+0,9(t+x)+0,3(t+x)^{2}\right) \\
-e^{-t}\left(1+0,9 t+0,3 t^{2}\right) e^{-x}\left(1+0,9 x+0,3 x^{2}\right) \\
=1+0,9 t+0,9 x+0,3 t^{2}+0,6 t x+0,3 x^{2} \\
-\left(1+0,9 x+0,3 x^{2}+0,9 t+0,81 t x+0,27 t x^{2}+0,3 t^{2}+0,27 t^{2} x+0,09 t^{2} x^{2}\right) \\
=-0,21 t x-0,27 t^{2} x-0,27 t x^{2}-0,09 t^{2} x^{2} \leq 0 .
\end{gathered}
$$

Concluímos que T é NBU.

Exemplo A.4.12. Verificação do Exemplo 3.1.9.

Segundo o Exemplo 3.1.7, o D-espectro f é NWU.

A confiabilidade do network no instante $\mathrm{t}>0$ é

$$
\bar{H}(t)=e^{-t^{2}}\left(1+0,5 \cdot t^{2}+0,15 \cdot t^{4}\right) .
$$

Tome $t_{3}=1, t_{1}=0,3$ e $t_{2}=0,7$.

Assim,

$$
P(T>1)-P(T>0,3) \cdot P(T>0,7)=0,60-1,41=-0,81<0 .
$$

Então, existem $t_{3}=1, t_{1}=0,3$ e $t_{2}=0,7$, com $t_{3}=t_{1}+t_{2}$ tais que

$$
P\left(T>t_{3}\right)-P\left(T>t_{1}\right) \cdot P\left(T>t_{2}\right) \leq 0 .
$$

Observe que, para todo $\mathrm{x}, \mathrm{y}>0, \Lambda(x+y)=(x+y)^{2}=x^{2}+y^{2}+2 . x . y \geq x^{2}+y^{2}=\Lambda(x)+\Lambda(y)$ e, portanto, a função de risco $\Lambda(t)=t^{2}$ é superaditiva. 
Concluímos que T não é NWU, contrariamente à classe do D-espectro $\mathbf{f}$.

Exemplo A.4.13. Verificação do Exemplo 3.1.10.

Analisamos a preservação da classe NBUE (NWUE). Observe que

(i) $\mu=\sum_{j=1}^{3} \bar{F}_{j}=1,8<\infty$;

(ii) Avaliamos o sinal de $\sum_{j=k}^{3} \bar{F}_{j-1}-\mu \cdot \bar{F}_{k}$ para $\mathrm{k}=1,2,3$.

Se $\mathrm{k}=1$, então $\sum_{j=1}^{3} \bar{F}_{j-1}-\mu \cdot \bar{F}_{1}=1,8-1,8.0,5=0,9>0$;

Se k $=2$, então $\sum_{j=2}^{3} \bar{F}_{j-1}-\mu \cdot \bar{F}_{2}=0,8-1,8.0,3=0,26>0$;

Se $\mathrm{k}=3$, então $\sum_{j=3}^{3} \bar{F}_{j-1}-\mu \cdot \bar{F}_{3}=0,3-1,8.0=0,3>0$.

Assim, $\sum_{j=k}^{3} \bar{F}_{j-1}-\mu \cdot \bar{F}_{k}>0$, para $\mathrm{k}=1,2,3$.

Portanto, o D-espectro f é NWUE.

Exemplo A.4.14. Verificação do Exemplo 3.1.11.

Do Exemplo 3.1.8, sabemos que f é NBU, o que implica, pelo Teorema A.3.3, que f é NBUE.

Observe que

$$
\frac{\Lambda(t+x)}{t+x}-\frac{\Lambda(t)}{t}=\frac{\lambda \cdot(t+x)}{t+x}-\frac{\lambda \cdot(t)}{t}=\lambda-\lambda=0,
$$

isto é, a função de risco $\Lambda(t)=\lambda . t$ é star-shaped e antistar-shaped.

A confiabilidade do network é

$$
\bar{H}(t)=\sum_{k=0}^{2} \bar{F}_{k} \cdot P(N(t)=k)=e^{-t}\left(1+0,9 t+0,3 t^{2}\right) .
$$

O tempo de vida médio do network é

$$
\begin{gathered}
\mu=E(T)=\int_{0}^{\infty} \bar{H}(t) d t=\int_{0}^{\infty} e^{-t}\left(1+0,9 t+0,3 t^{2}\right) d t=\int_{0}^{\infty} e^{-t} d t+\int_{0}^{\infty} 0,9 t e^{-t} d t+\int_{0}^{\infty} 0,3 t^{2} e^{-t} d t \\
=1+0,9+0,6=2,5 .
\end{gathered}
$$

A segunda integral resulta de uma integração por partes e a terceira como integração da função densidade Gama(3,1).

Vamos veirificar que $\bar{H}(t) . \mu \geq \int_{t}^{\infty} \bar{H}(s) d s$. Observe que

$$
\begin{gathered}
\int_{t}^{\infty} \bar{H}(s) d s=\int_{t}^{\infty} e^{-s}\left(1+0,9 s+0,3 s^{2}\right) d s=\int_{t}^{\infty} e^{-s} d s+\int_{t}^{\infty} 0,9 s e^{-s} d s+\int_{t}^{\infty} 0,3 s^{2} e^{-s} d s \\
=e^{-t}+0,9 \cdot e^{-t}(1+t)+0,3 e^{-t}\left(1+2 t+2 t^{2}\right)=e^{-t}\left(2,5+1,5 \cdot t+0,3 \cdot t^{2}\right)=2,5 \cdot e^{-t}\left(1+0,6 . t+0,12 . t^{2}\right) \\
\leq \mu \cdot e^{-t}\left(1+0,9 t+0,3 t^{2}\right)=\mu \cdot \bar{H}(t) .
\end{gathered}
$$

A segunda e a terceira integrais resultam de integrações por partes, sendo uma para a segunda e duas para a terceira integral.

Concluímos que T é NBUE.

Exemplo A.4.15. Verificação do Exemplo 3.1.12. 
Queremos avaliar a preservação da classe de distribuição NBUE (NWUE).

Observe que

$$
\frac{d \Lambda(t)}{d t}=\frac{d}{d t} \frac{e^{t}}{t}=\frac{e^{t} \cdot t-e^{t}}{t^{2}}=\frac{e^{t}}{t^{2}}(t-1) .
$$

Concluímos que $\frac{d \Lambda(t)}{d t} \geq(\leq) 0 \Longleftrightarrow t \geq(\leq) 1$.

Portanto, $\Lambda(t)$ é antistar-shaped para $\mathrm{t} \leq 1$ e star-shaped para $t \geq 1$.

Segundo o Exemplo 3.1.10, o D-espectro f é NWUE.

A confiabilidade do network no instante $\mathrm{t}>0$ é

$$
\bar{H}(t)=e^{-e^{t}} \cdot\left(1+0,5 \cdot e^{t}+0,15 \cdot e^{2 t}\right) .
$$

Verificamos a parte (i) da classe NBUE (NWUE)

O tempo de vida médio do network é

$$
\mu=E(T)=\int_{0}^{\infty} \bar{H}(t) d t=\int_{0}^{\infty} e^{-e^{t}} \cdot\left(1+0,5 . e^{t}+0,15 . e^{2 t}\right) d t .
$$

Aplicando a mudança de variável $v=e^{t}$, temos

$$
\begin{gathered}
\mu=\int_{1}^{\infty} e^{-v} \cdot\left(1+0,5 \cdot v+0,15 \cdot v^{2}\right) \frac{d v}{v} \\
=E i(-1)+0,8 \cdot e^{-1} \approx 0,22+0,8 \cdot e^{-1} \approx 0,51<\infty,
\end{gathered}
$$

onde $\operatorname{Ei}($.$) é a Função Exponencial Integral (Definição A.3.9).$

(ii) Considere $t_{0}=2$. Então

$$
\begin{gathered}
\bar{H}(2)=e^{-e^{2}}\left(1+0,5 \cdot e^{2}+0,15 \cdot e^{4}\right) \approx 0,08 \\
\int_{2}^{\infty} \bar{H}(t) d t=\int_{2}^{\infty} e^{-e^{t}} \cdot\left(1+0,5 . e^{t}+0,15 . e^{2 t}\right) d t .
\end{gathered}
$$

Aplicando a mudança de variável $\mathrm{v}=e^{t}$, temos

$$
\bar{H}(2)=\int_{e^{2}}^{\infty} e^{-v} \cdot\left(1+0,5 \cdot v+0,15 \cdot v^{2}\right) \frac{d v}{v}=E i\left(-e^{2}\right)+0,001 \approx 0,00107 .
$$

Portanto

$$
\int_{2}^{\infty} \bar{H}(t) d t-\mu \cdot \bar{H}(2)=0,00107-0,51.0,008 \approx-0,003<0 .
$$

Então existe $t_{0}=2$ tal que

$$
\int_{2}^{\infty} \bar{H}(t) d t-\mu \cdot \bar{H}(2)<0 .
$$

Concluímos que T não é NWU, contrariamente ao D-espectro f.

Exemplo A.4.16. Verificação do Exemplo 3.2.1.

Seja $\bar{F}_{k}$ a função de sobrevivência do D-espectro $\mathbf{f}$. 
Temos: $\bar{F}_{0}=1 \bar{F}_{1}=0,9 \bar{F}_{2}=0,6$.

Verificaremos se $\mathbf{f}$ é IFR, ou seja, para todo $\mathrm{k}, \mathrm{l}=1,2, \ldots 5$

$$
\frac{\bar{F}_{k+l}}{\bar{F}_{k}} \geq \frac{\bar{F}_{k+1+l}}{\bar{F}_{k+1}} \Longleftrightarrow \bar{F}_{k+l} \cdot \bar{F}_{k+1}-\bar{F}_{k+1+l} \cdot \bar{F}_{k} \geq 0 .
$$

Concluímos que $\bar{F}_{2}^{2}-\bar{F}_{1} \cdot \bar{F}_{3}=0,9^{2}-1.0,3=0,21 \geq 0$.

Portanto $\bar{F}_{k+l} \cdot \bar{F}_{k+1}-\bar{F}_{k+1+l} \cdot \bar{F}_{k} \geq 0$, para todo $\mathrm{k}, 1=1,2, \ldots 5$ e f é IFR. Segundo o Teorema A.3.3, f é IFRA, NBU e NBUE.

O k-ésimo termo do D-espectro condicional em t é

$$
p_{k}(t)=\frac{f_{k} \cdot P\left(R_{k}>t\right)}{P(T>t)} .
$$

A confiabilidade do network é

$$
P(T>t)=e^{-t^{2}}\left(1+0,9 t^{2}+\frac{0,6}{2} t^{4}\right)=e^{-t^{2}}\left(1+0,9 t^{2}+0,3 t^{4}\right) .
$$

Assim

$$
\begin{gathered}
P\left(R_{1}>t\right)=e^{-t^{2}} ; \\
P\left(R_{2}>t\right)=e^{-t^{2}}\left(1+t^{2}\right) ; \\
P\left(R_{3}>t\right)=e^{-t^{2}}\left(1+t^{2}+\frac{t^{4}}{2}\right) .
\end{gathered}
$$

Portanto

$$
\begin{aligned}
p_{1}(t) & =\frac{0,1}{1+0,9 t^{2}+0,3 t^{4}} \\
p_{2}(t) & =\frac{0,3+0,3 t^{2}}{1+0,9 t^{2}+0,3 t^{4}} \\
p_{3}(t) & =\frac{0,6+0,6 t^{2}+0,3 t^{4}}{1+0,9 t^{2}+0,3 t^{4}} .
\end{aligned}
$$

Além disso

$$
\begin{gathered}
\sum_{k=1}^{3} p_{k}(t)=1 \\
\sum_{k=2}^{3} p_{k}(t)=\frac{0,3+0,3 t^{2}}{1+0,9 t^{2}+0,3 t^{4}}+\frac{0,6+0,6 t^{2}+0,3 t^{4}}{1+0,9 t^{2}+0,3 t^{4}}=\frac{0,9+0,9 t^{2}+0,3 t^{4}}{1+0,9 t^{2}+0,3 t^{4}} \\
\sum_{k=3}^{3} p_{k}(t)=p_{3}(t)=\frac{0,6+0,6 t^{2}+0,3 t^{4}}{1+0,9 t^{2}+0,3 t^{4}}
\end{gathered}
$$

Verificamos que $\mathbf{p}(\mathbf{t})$ é IFR, ou seja, $\frac{\sum_{k=j+1}^{3} p_{k}(t)}{\sum_{k=j}^{3} p_{k}(t)}$ é decrescente em j, ou seja,

$$
\left(\sum_{k=j}^{3} p_{k}(t)\right)^{2}-\sum_{k=j-1}^{3} p_{k}(t) . \sum_{k=j+1}^{3} p_{k}(t) \geq 0 .
$$


Então

$$
\begin{aligned}
& \left(\sum_{k=2}^{3} p_{k}(t)\right)^{2}-\sum_{k=1}^{3} p_{k}(t) \cdot \sum_{k=3}^{3} p_{k}(t)=\left(\frac{0,9+0,9 t^{2}+0,3 t^{4}}{1+0,9 t^{2}+0,3 t^{4}}\right)^{2}-1 \cdot \frac{0,6+0,6 t^{2}+0,3 t^{4}}{1+0,9 t^{2}+0,3 t^{4}} \\
= & \frac{0,81+1,62 t^{2}+1,35 t^{4}+0,54 t^{6}+0,09 t^{8}}{\left(1+0,9 t^{2}+0,3 t^{4}\right)^{2}}-\frac{\left(0,6+0,6 t^{2}+0,3 t^{4}\right)\left(1+0,9 t^{2}+0,3 t^{4}\right)}{\left(1+0,9 t^{2}+0,3 t^{4}\right)^{2}} \\
= & \frac{0,81+1,62 t^{2}+1,35 t^{4}+0,54 t^{6}+0,09 t^{8}}{\left(1+0,9 t^{2}+0,3 t^{4}\right)^{2}}-\frac{0,6+1,14 t^{2}+1,02 t^{4}+0,45 t^{6}+0,09 t^{8}}{\left(1+0,9 t^{2}+0,3 t^{4}\right)^{2}} \\
= & \frac{0,21+0,48 t^{2}+0,33 t^{4}+0,09 t^{6}}{1+0,9 t^{2}+0,3 t^{4}} \geq 0 .
\end{aligned}
$$

Concluímos que $\mathbf{p}(\mathbf{t})$ é IFR. Isto implica, segundo o Teorema A.3.3, que $p(t)$ é IFRA.

Verificamos que $\mathbf{p}(\mathbf{t})$ é NBU, isto é

$$
\sum_{k=3}^{3} p_{k}(t)-\sum_{k=1}^{3} p_{k}(t) \cdot \sum_{k=2}^{3} p_{k}(t)=\frac{0,6+0,6 t^{2}+0,3 t^{4}}{1+0,9 t^{2}+0,3 t^{4}}-1 \cdot \frac{0,9+0,9 t^{2}+0,3 t^{4}}{1+0,9 t^{2}+0,3 t^{4}} \leq 0
$$

Portanto $\mathbf{p}(\mathbf{t})$ é NBU.

Verificaremos que $\mathbf{p}(\mathbf{t})$ é NBUE, ou seja $\sum_{k=j}^{3} p_{k}(t) . \mu(t)-\sum_{k=j}^{3} \sum_{l=k}^{3} p_{l}(t) \geq 0$, onde

$$
\mu(t)=\sum_{j=1}^{3} \sum_{k=j}^{3} p_{k}(t)=\frac{2,5+1,8 t^{2}+0,9 t^{4}}{1+0,9 t^{2}+0,3 t^{4}}
$$

Portanto

$$
\begin{gathered}
\sum_{k=2}^{3} p_{k}(t) \cdot \mu(t)-\sum_{k=2}^{3} \sum_{l=k}^{3} p_{l}(t)=\frac{0,9+0,9 t^{2}+0,3 t^{4}}{1+0,9 t^{2}+0,3 t^{4}} \cdot \frac{2,5+1,8 t^{2}+0,9 t^{4}}{1+0,9 t^{2}+0,3 t^{4}}-\frac{1,5+1,5 t^{2}+0,6 t^{4}}{1+0,9 t^{2}+0,3 t^{4}} \\
=\frac{0,75+1,02 t^{2}+0,78 t^{4}+0,36 t^{6}+0,09 t^{8}}{\left(1+0,9 t^{2}+0,3 t^{4}\right)^{2}} \geq 0 .
\end{gathered}
$$

Portanto $\mathbf{p}(\mathbf{t})$ é NBUE.

Exemplo A.4.17 Verificação do Exemplo 3.3.1.

Nosso interesse é analisar a preservação das classes de distribuições do D-espectro condicional $\mathbf{p}(\mathbf{t})$ para o tempo de vida do network.

Seja $\bar{F}=\left(\bar{F}_{0}, \bar{F}_{1}, \bar{F}_{2}\right)$, onde $\bar{F}_{k}$ é a função de sobrevivência do D-espectro $\mathbf{f}$ a k falhas dos links.

A confiabilidade do network no instante t é

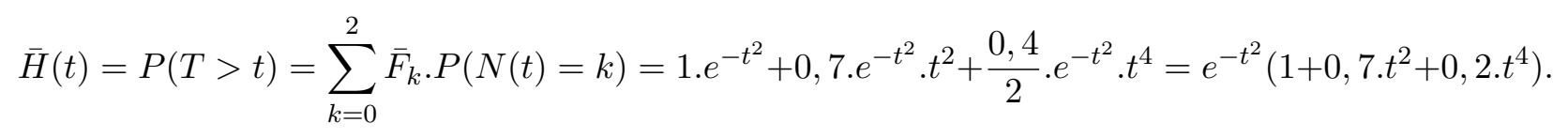

O k-ésimo componente do D-espectro condicional do network é

$$
p_{k}(t)=\frac{f_{k} \cdot P\left(R_{k}>t\right)}{\bar{H}(t)},
$$


onde $R_{k}$ é o instante da k-ésima falha dos links.

Verificaremos que $\mathbf{p}(\mathbf{t})$ tem taxa de falha crescente, isto é, $\frac{p_{k}(t)}{\sum_{j=k}^{n} p_{j}(t)}$ é crescente em $\mathrm{k}=1,2,3,4$.

Temos:

$$
\begin{gathered}
p_{1}(t)=\frac{f_{1} \cdot P\left(R_{1}>t\right)}{\bar{H}(t)}=\frac{0,3 \cdot e^{-t^{2}}}{e^{-t^{2}}\left(1+0,7 \cdot t^{2}+\frac{0,2}{\cdot} t^{4}\right)}=\frac{0,3}{1+0,7 \cdot t^{2}+0,2 \cdot t^{4}} ; \\
p_{2}(t)=\frac{f_{2} \cdot P\left(R_{2}>t\right)}{\bar{H}(t)}=\frac{0,3 \cdot\left(e^{-t^{2}}+e^{-t^{2}} \cdot t^{2}\right)}{e^{-t^{2}}\left(1+0,7 \cdot t^{2}+0,2 \cdot t^{4}\right)}=\frac{0,3+0,3 \cdot t^{2}}{1+0,7 \cdot t^{2}+0,2 \cdot t^{4}} \\
p_{3}(t)=\frac{f_{3} \cdot P\left(R_{3}>t\right)}{\bar{H}(t)}=\frac{0,4 \cdot\left(e^{-t^{2}}+e^{-t^{2}} \cdot t^{2}+e^{-t^{2}} \cdot \frac{t^{4}}{2}\right)}{e^{-t^{2}}\left(1+0,7 \cdot t^{2}+0,2 \cdot t^{4}\right)}=\frac{0,4+0,4 \cdot t^{2}+0,2 \cdot t^{4}}{1+0,7 \cdot t^{2}+0,2 \cdot t^{4}} .
\end{gathered}
$$

A função de sobrevivência do D-espectro condicional para os valores de k é

$$
\begin{gathered}
\sum_{j=1}^{3} p_{j}(t)=1 \\
\sum_{j=2}^{3} p_{j}(t)=\frac{0,7+0,7 \cdot t^{2}+0,2 \cdot t^{4}}{1+0,7 \cdot t^{2}+0,2 . t^{4}} \\
\sum_{j=3}^{3} p_{j}(t)=\frac{0,4+0,4 \cdot t^{2}+0,2 \cdot t^{4}}{1+0,7 \cdot t^{2}+0,2 \cdot t^{4}} .
\end{gathered}
$$

A primeira igualdade vale, pois o D-espectro condicional é uma função discreta de probabilidades para cada $t>0$, fixado.

As taxas de falhas para os componentes do D-espectro condicional em t são

$$
\begin{gathered}
r_{1}(t)=\frac{p_{1}(t)}{\sum_{j=1}^{3} p_{j}(t)}=\frac{\frac{0,3}{1+0,7 \cdot t^{2}+0,2 \cdot t^{4}}}{1}=\frac{0,3}{1+0,7 \cdot t^{2}+0,2 \cdot t^{4}} ; \\
r_{2}(t)=\frac{p_{2}(t)}{\sum_{j=2}^{3} p_{j}(t)}=\frac{\frac{0,3+0,3 \cdot t^{2}}{1+0,7 \cdot t^{2}+0,2 \cdot t^{4}}}{\frac{0,7+0,7 \cdot t^{2}+0,2 \cdot t^{4}}{1+0,7 \cdot t^{2}+0,2 \cdot t^{4}}}=\frac{0,3+0,3 \cdot t^{2}}{0,7+0,7 \cdot t^{2}+0,2 \cdot t^{4}} ; \\
r_{3}(t)=\frac{p_{3}(t)}{\sum_{j=3}^{3} p_{j}(t)}=\frac{\frac{0,4+0,4 \cdot t^{2}+0,2 \cdot t^{4}}{1+0,7 \cdot t^{2}+0,2 \cdot t^{4}}}{\frac{0,4+0,4 \cdot t^{2}+0,2 \cdot t^{4}}{1+0,7 \cdot t^{2}+0,2 \cdot t^{4}}}=1 .
\end{gathered}
$$

Observe que $r_{1}(t) \leq r_{2}(t)$, pois $0,3 \leq 0,3+0,3 . t^{2}$ e $1+0,7 . t^{2}+0,2 . t^{4} \geq 0,7+0,7 . t^{2}+0,2 . t^{4}$. É imediato concluir que $r_{2}(t) \leq r_{3}(t)$.

Portanto, o D-espectro condicional $\mathbf{p}(\mathbf{t})$ é IFR. Pelo Teorema A.3.3, $\mathbf{p}(\mathbf{t})$ também é IFRA, NBU e NBUE.

Observe que $\frac{d^{2} \Lambda(t)}{d t^{2}}=\frac{d^{2} t^{2}}{d t^{2}}=2>0$, e, portanto, a função de risco $\Lambda(t)=t^{2}$ é côncava.

Note que $\frac{\Lambda(t)}{t}=\frac{t^{2}}{t}=t$ e $\frac{d}{d t} \frac{\Lambda(t)}{t}=\frac{d}{d t} \frac{t^{2}}{t}=1>0$, isto é, $\frac{\Lambda(t)}{t}$ é crescente em t $>0$. Portanto, $\Lambda(t)=t^{2}$ é star-shaped.

Observe que $\Lambda(t+x)=(t+x)^{2}=t^{2}+x^{2}+2 . t . x \geq t^{2}+x^{2}=\Lambda(t)+\Lambda(x)$, ou seja, a função de risco é superaditiva. 
Verificamos a logconcavidade de $\bar{H}(t)$. Temos:

$$
\begin{gathered}
\log \bar{H}(t)=-t^{2}+\log \left(1+0,7 t^{2}+0,2 t^{4}\right) ; \\
\frac{d}{d t} \log \bar{H}(t)=-2 t+\frac{1,4 t+0,8 t^{3}}{1+0,7 t^{2}+0,2 t^{4}} ; \\
\frac{d^{2}}{d t^{2}} \log \bar{H}(t)=-2+\frac{\left(1,4+2,4 t^{2}\right)\left(1+0,7 t^{2}+0,2 t^{4}\right)}{\left(1+0,7 t^{2}+0,2 t^{4}\right)^{2}}-\frac{\left(1,4 t+0,8 t^{3}\right)\left(1,4 t+0,8 t^{3}\right)}{\left(1+0,7 t^{2}+0,4 t^{4}\right)^{2}} \\
=-2+\frac{1,4+0,98 t^{2}+0,28 t^{4}+2,4 t^{2}+1,68 t^{4}+0,48 t^{6}-1,96 t^{2}-2,24 t^{4}-0,64 t^{6}}{\left(1+0,7 t^{2}+0,4 t^{4}\right)^{2}} \\
=\frac{-2\left(1+0,7 t^{2}+0,4 t^{4}\right)^{2}+1,4+1,42 t^{2}-0,28 t^{4}-0,16 t^{6}}{\left(1+0,7 t^{2}+0,4 t^{4}\right)^{2}} \\
=\frac{-0,6-1,38 t^{2}-1,5 t^{4}-0,4 t^{6}-0,32 t^{8}}{\left(1+0,7 t^{2}+0,4 t^{4}\right)^{2}} \leq 0 .
\end{gathered}
$$

Concluímos que $\bar{H}(t)$ é log-côncava e T é IFR. Usando o Teorema A.3.3, isto implica que T é IFRA, NBU e NBUE. 
APÊNDICE A 


\section{Referências Bibliográficas}

A-Hameed e Proschan(1973) A-Hameed e Frank Proschan. Nonstationary shock models. Stochastic Processes and their Applications I, 1:383-404. Citado na pág. 3, 21, 25, 28, 31

Arnold et al.(1998) B.C. Arnold, N. Balakrishnan e H.N. Nagraja. Records. John Wiley and Sons. Citado na pág. 3,4

Balakrishnan e Asadi(2012) N. Balakrishnan e M. Asadi. A proposed measure of residual life of live components of a coherent system. IEEE Transactions on Reliability, 61:41-49. Citado na pág. 3

Barlow e Proschan(1975) Richard Barlow e F. Proschan. Statistical theory of reliability and life testing. To Begin With, Silver Spring, 7 edição. Citado na pág. 19, 23, 26, 29, 41, 43, 52

Boland et al.(2003) P.J. Boland, F.J. Samaniego e E.M. Vestrup. Linking dominations and signatures in network reliability. Mathematical and Statistical Methods in Reliability, páginas 89-103. Citado na pág. 3

Bueno(2015) Vanderlei Da Costa Bueno. Preservation of non-parametric distribution classes under a system signature point process. Em Signature Structural Reliability, 60th ISI - World Statistics Congress, Rio de Janeiro. Citado na pág. 41

Esary et al.(1973) Esary, Marshall e Frank Proschan. Shock models and wear processes. The Annals of Probability, 1:627-649. Citado na pág. 3, 21, 24, 27, 31

Gertsbakh e Shpungin(2011) I. Gertsbakh e Y. Shpungin. Network reliability and resilience. Springer-Verlag. Citado na pág. 3, 41

Gupta e Kirman(1988) R.C. Gupta e S.N.U.A. Kirman. Closure and monotonicity properties of nonhomogeneous poisson processes and record values. Probability in the Engineering and Informational Sciences, 2:475-484. Citado na pág. 3, 4

Joag-Dev et al.(1992) Kumar Joag-Dev, Subhash Kochar e Frank Proschan. A general composition theorem and its applications to certain partial orderings of distributions. Citado na pág. 50

Karlin(1968) Samuel Karlin. Total Positivity, volume 1. Stanford University Press. Citado na pág. 48,50

Randles e Wolfe(1979) Ronald H. Randles e Douglas A. Wolfe. Introduction to the Theory of Nonparametric Statistics, volume 25. Wiley Series on Personality Processes. Citado na pág. 46

Samaniego et al.(2009) Francisco J. Samaniego, N. Balakrishnan e Jorge Navarro. Dynamic signatures and their use in comparing the reliability of new and used systems. Wiley Periodicals, Inc, 56:577-591. Citado na pág. 3, 44, 45

Shaked e Shanthikumar(2007) M. Shaked e J. G. Shanthikumar. Stochastic Orders. SpringerVerlag. Citado na pág. 47, 49 
Zarezadeh e Asadi(2013) S. Zarezadeh e M. Asadi. Network reliability modeling under stochastic process of component failures. IEEE Transactions on Reliability, 62:917-929. Citado na pág. 2, 5, $6,13,15$

Zarezadeh et al.(2013) S. Zarezadeh, M. Asadi e N. Balakrishnan. Dynamic network reliability modeling under nonhomogeneous poisson processes of component failures. European Journal of Operational Research, 232:561-571. Citado na pág. 1, 2, 6, 7, 17 